1-Benzyl-3-(2,6-dimethylphenyl)-3-methyl-1-phenylurea 1a
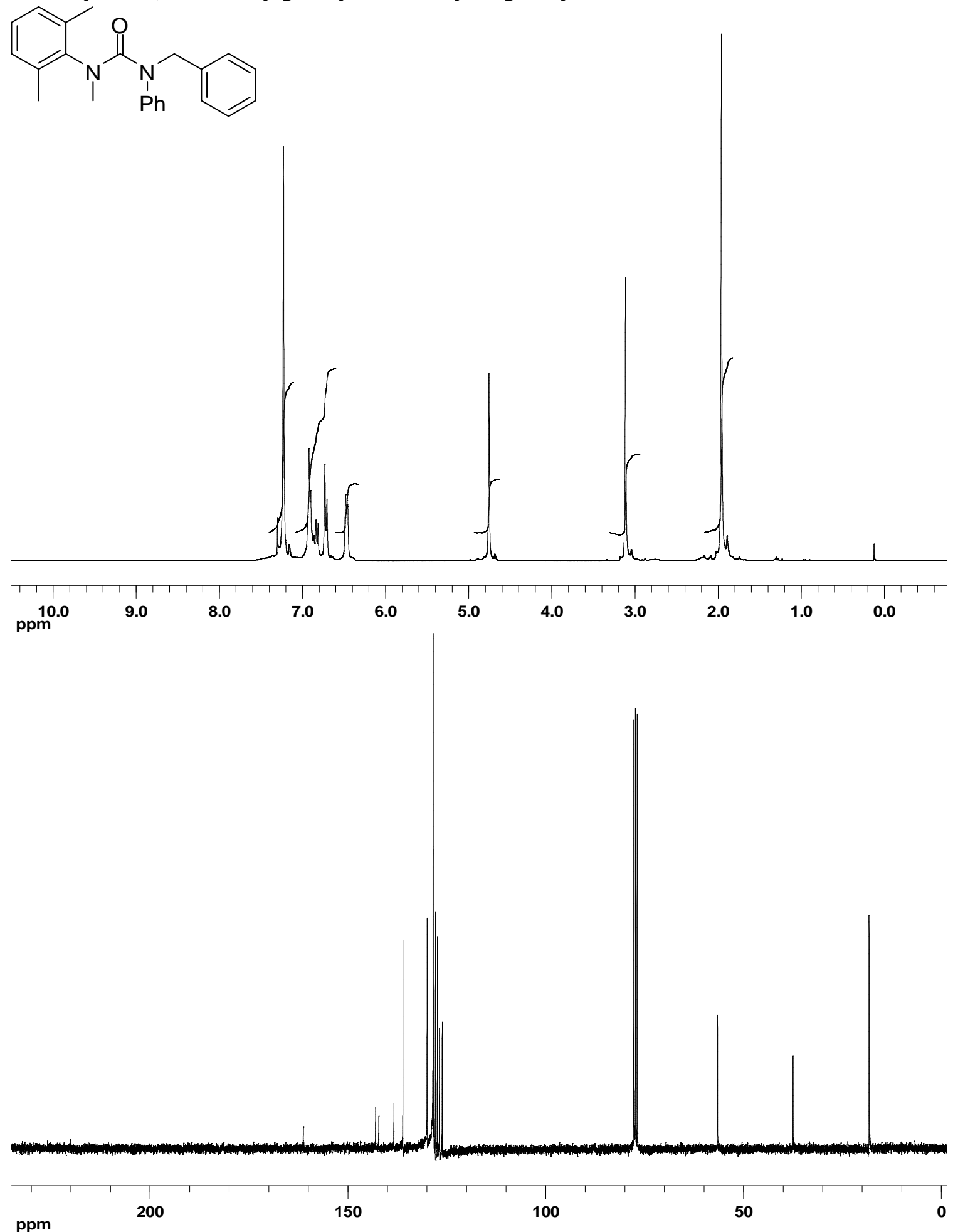


\section{1-Benzyl-3-methyl-1-phenyl-3-p-tolylurea 1b}<smiles>Cc1ccc(N(C)C(=O)N(Cc2ccccc2)c2ccccc2)cc1</smiles>
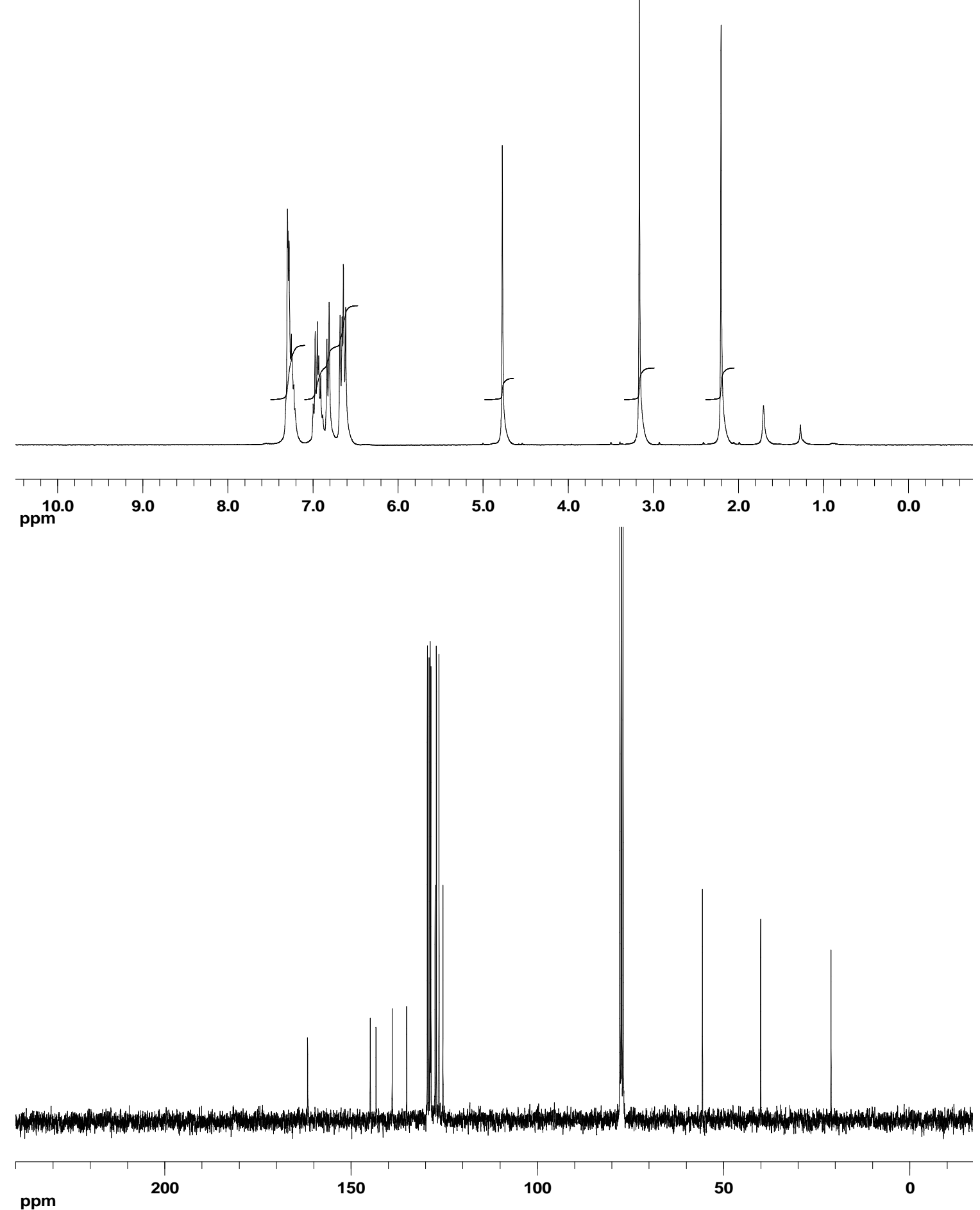
1-Benzyl-3-methyl-3-phenyl-1-p-tolylurea 1c
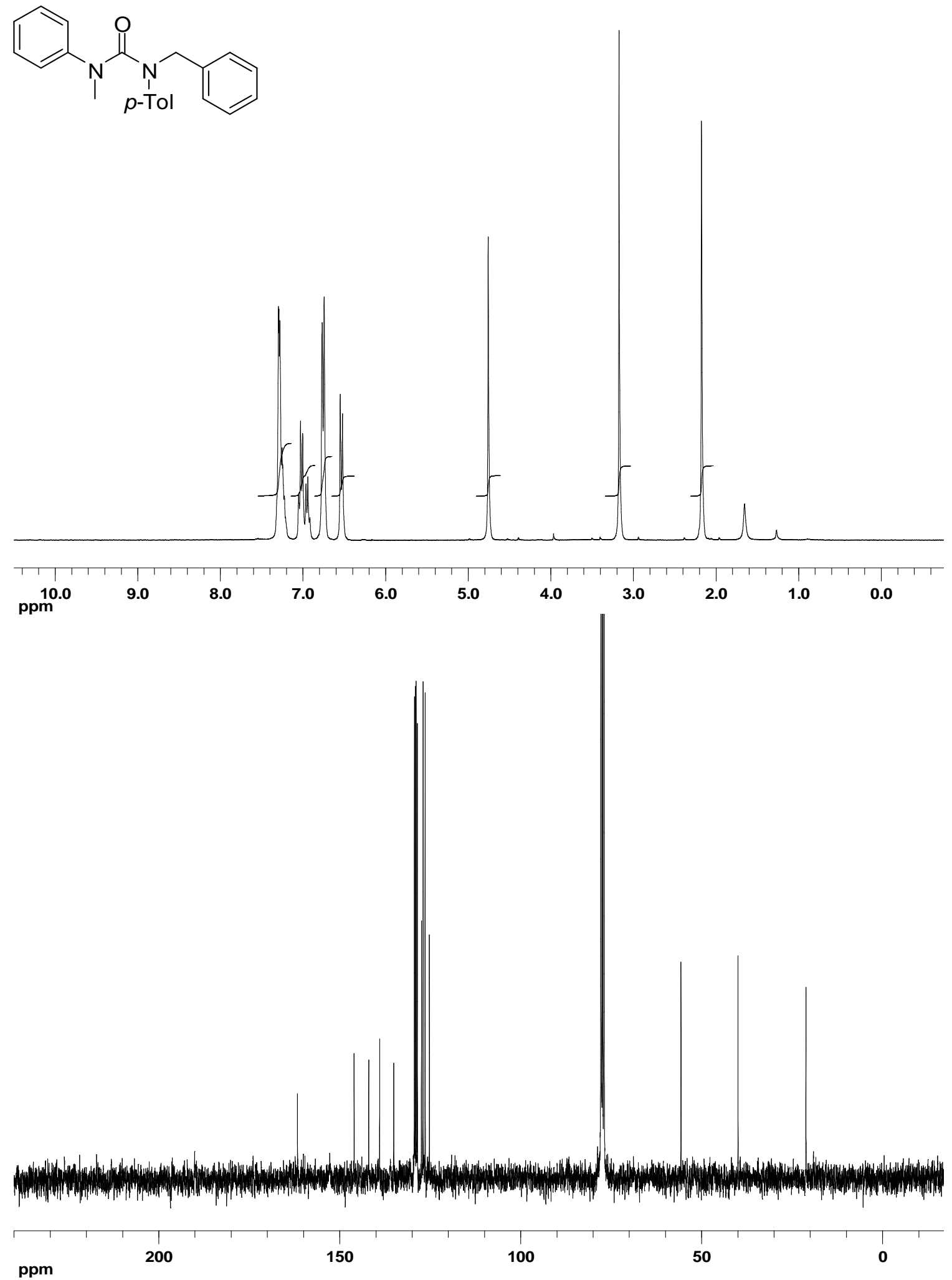
1-Benzyl-1,3-dimethyl-3-phenylurea 1d

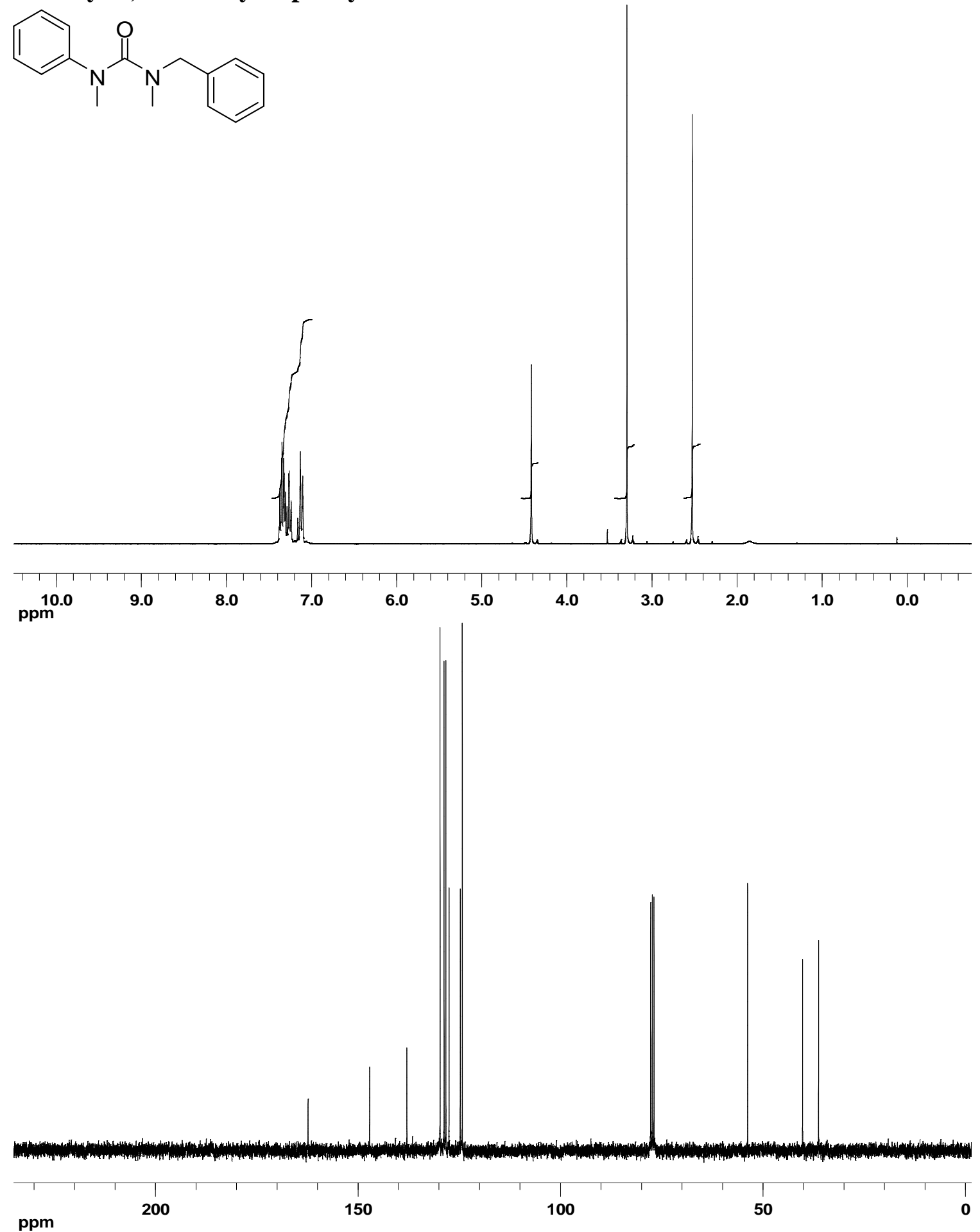




\section{1-Benzyl-1,3-dimethyl-3-o-tolylurea 1e}<smiles>Cc1ccccc1N(C)C(=O)N(C)Cc1ccccc1</smiles>
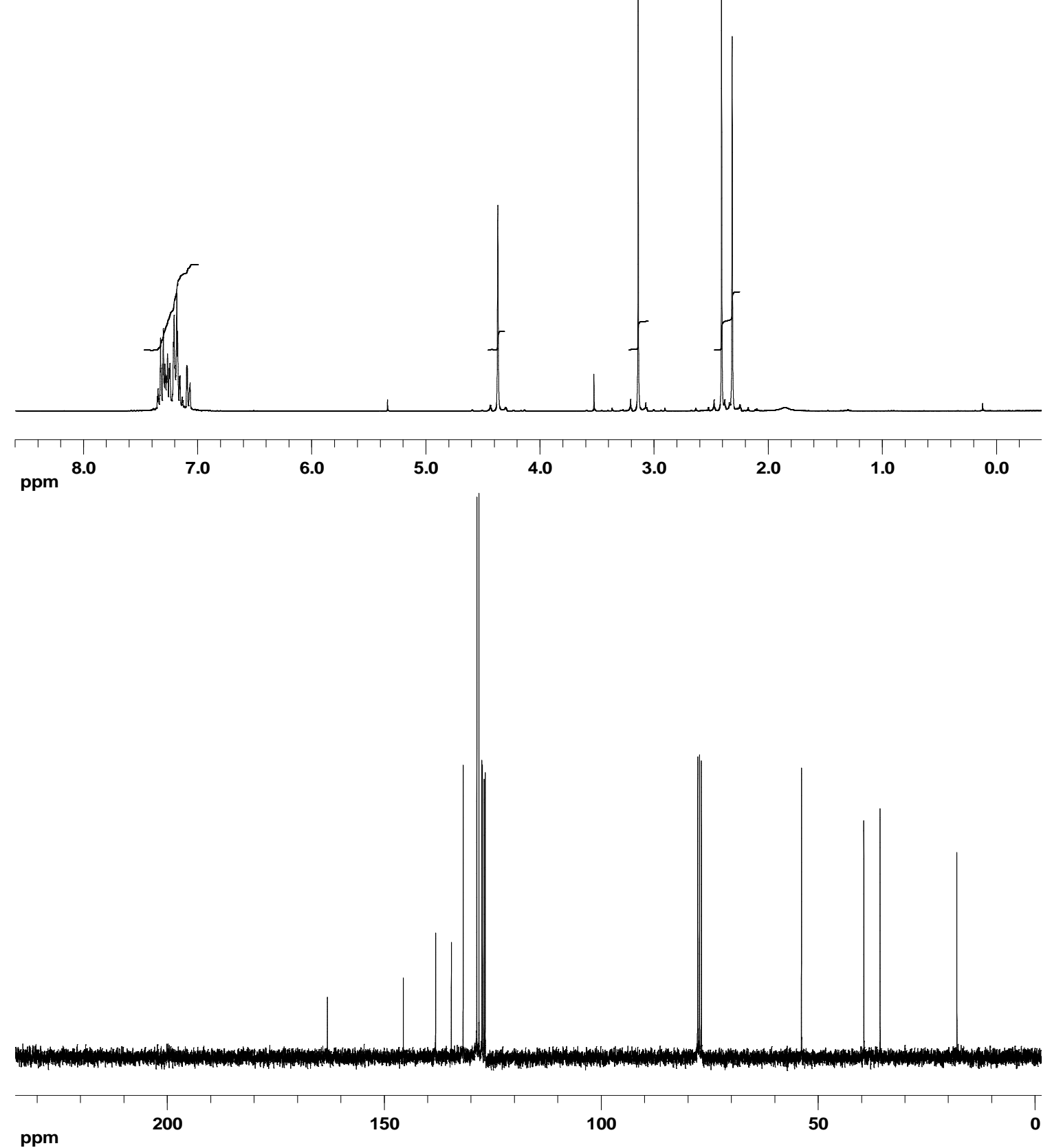
1-Benzyl-3-(2-methoxyphenyl)-1,3-dimethylurea if<smiles>COc1ccccc1N(C)C(=O)N(C)Cc1ccccc1</smiles>
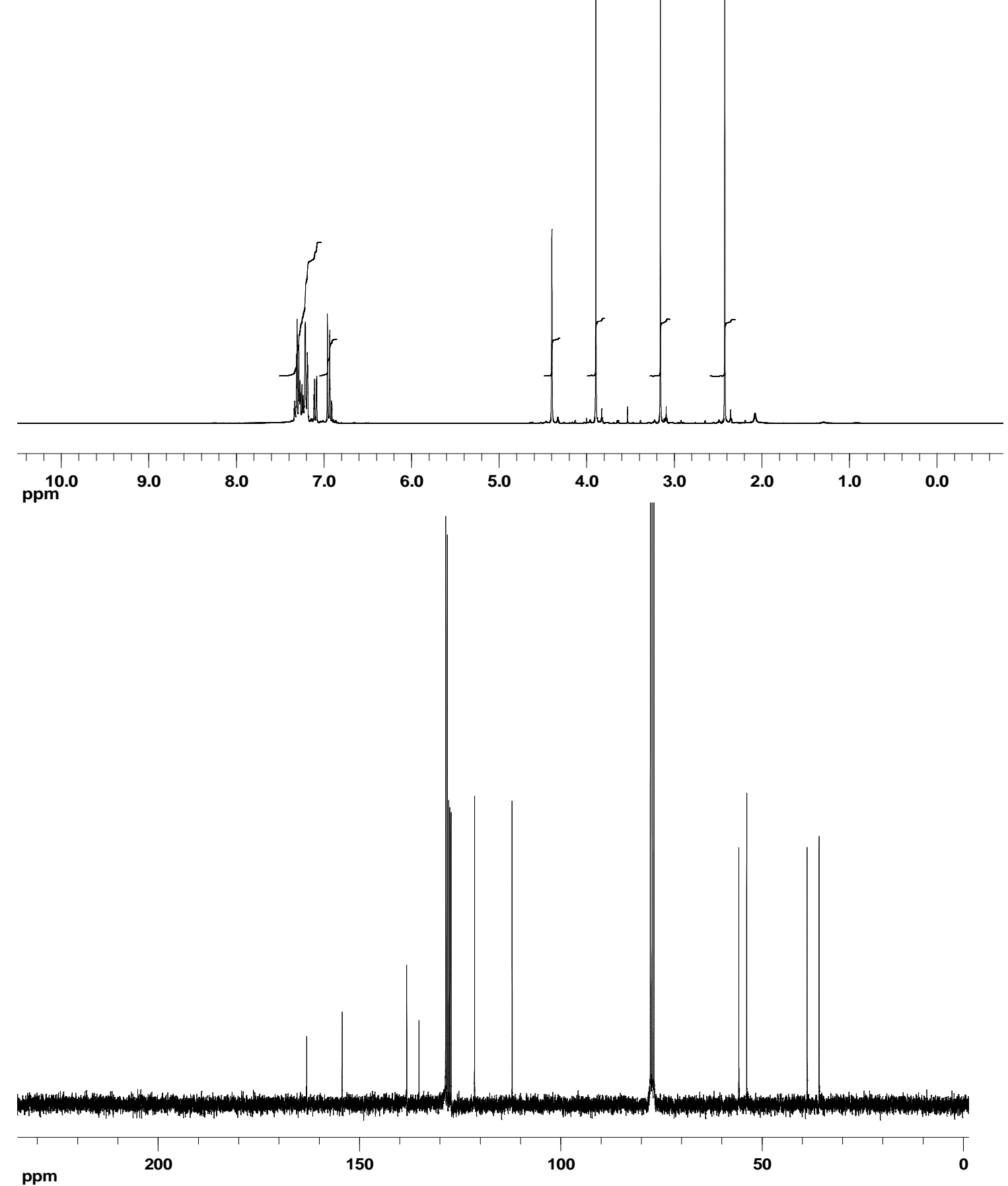
1-Benzyl-3-(2,6-dimethylphenyl)-1,3-dimethylurea $1 \mathrm{~g}$
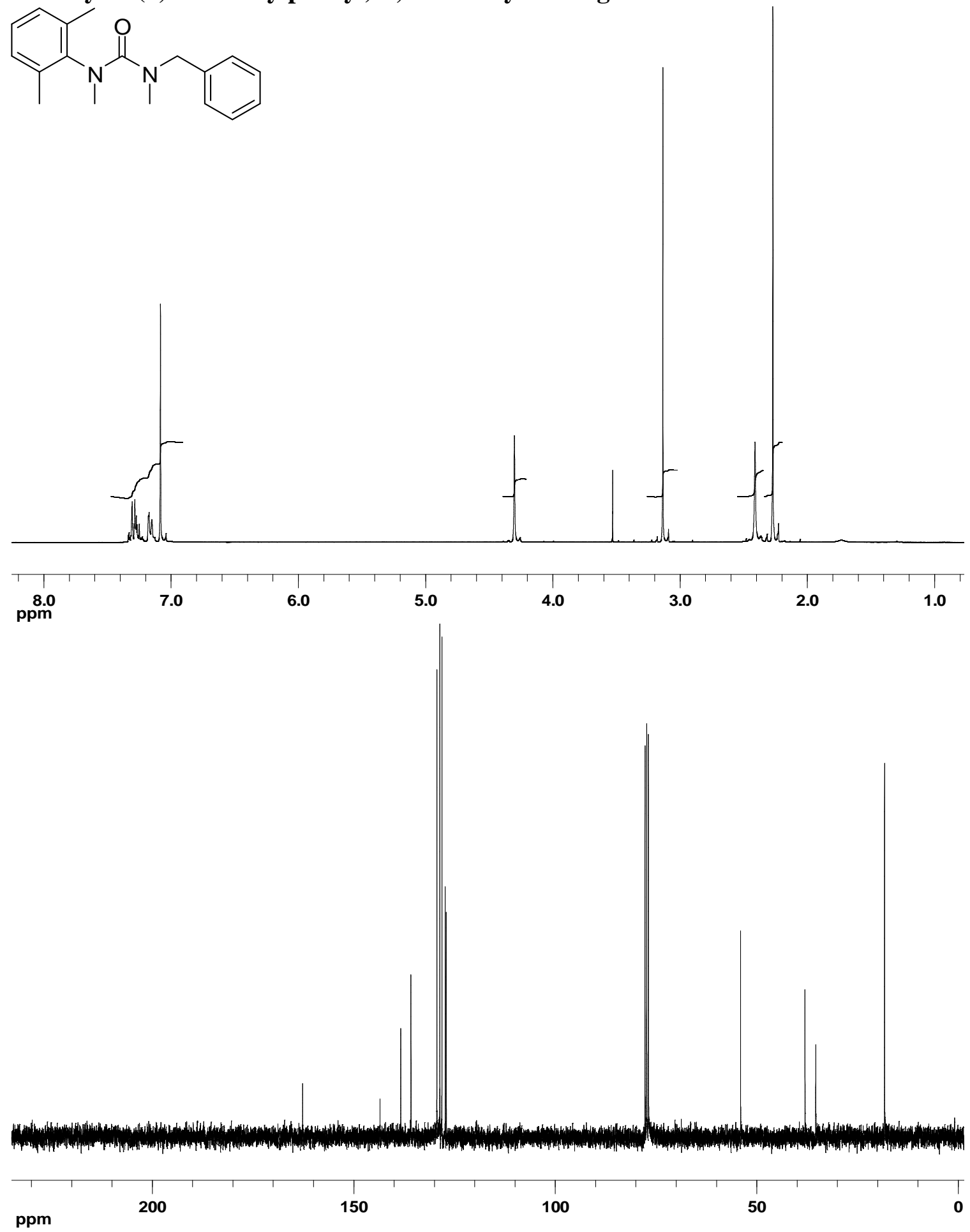


\section{1-Benzyl-3-(4-chlorophenyl)-1,3-dimethylurea $1 \mathrm{~h}$}

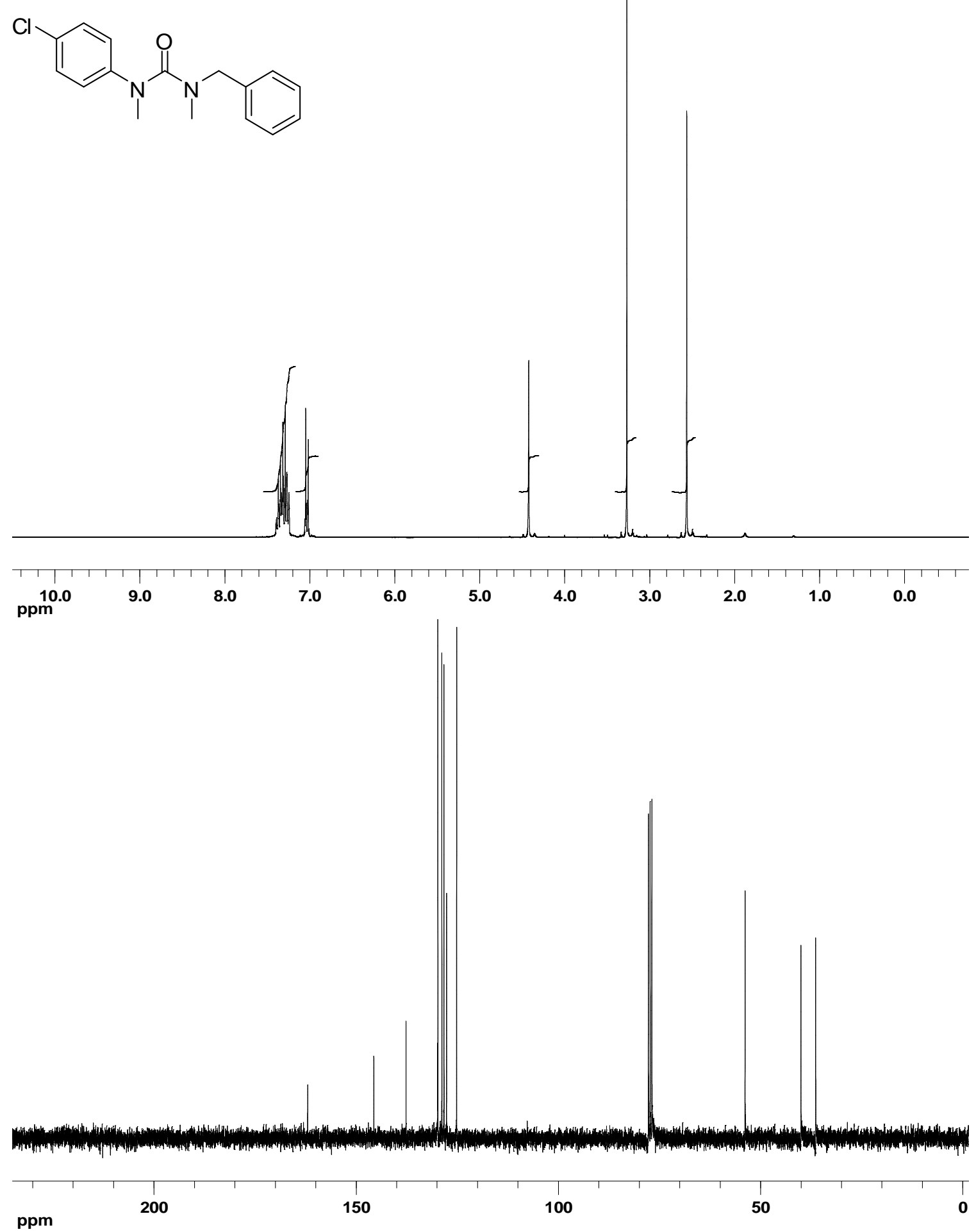


1-Benzyl-3-(4-methoxyphenyl)-1,3-dimethylurea 1i
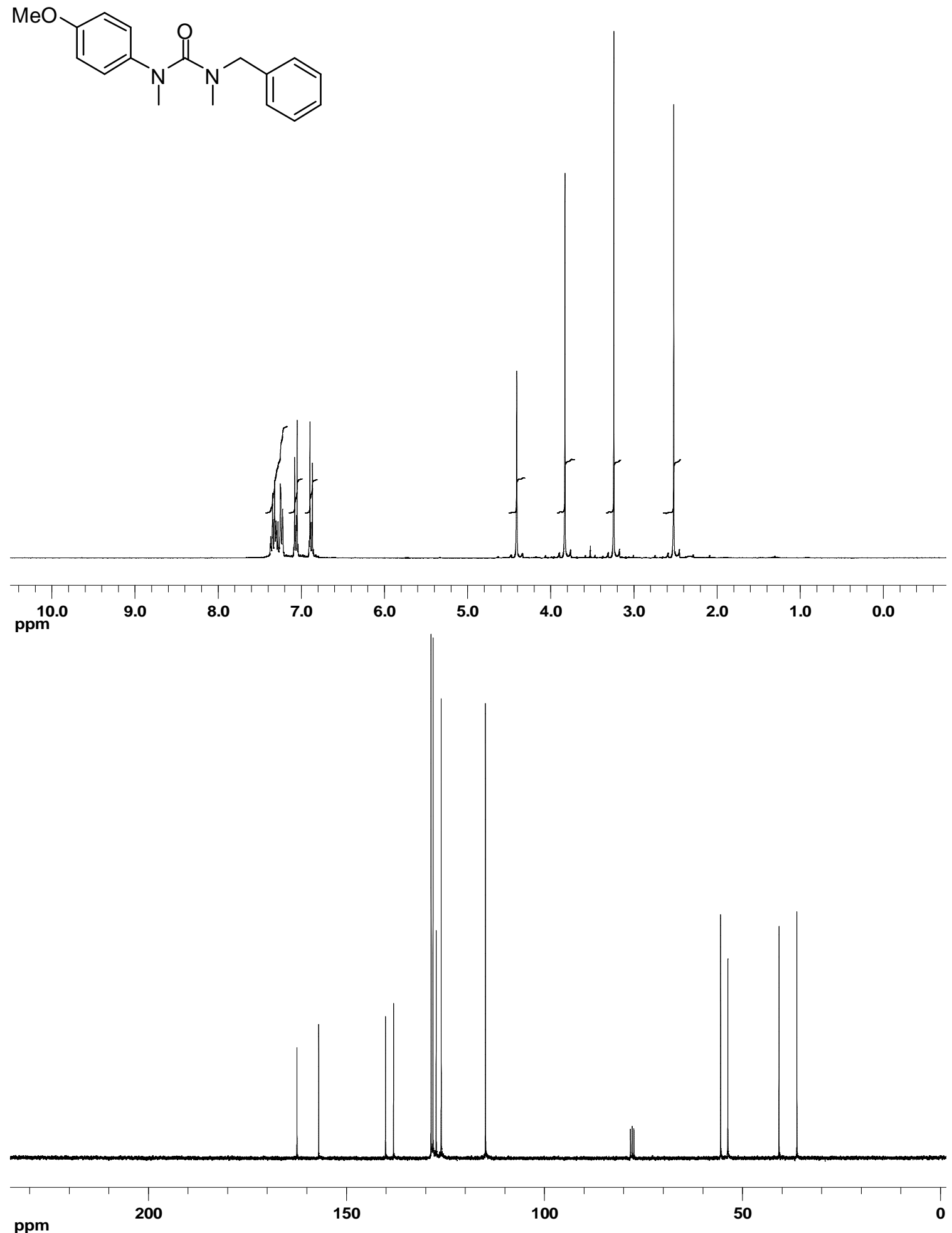
1-((2,6-Dimethylphenyl)(4-methylcyclohexa-2,5-dienylidene)methyl)-3,3-dimethyl-1phenylurea 2<smiles>Cc1cccc(C)c1C(=C1C=CC(C)C=C1)N(P)C(=O)N(C)C</smiles>
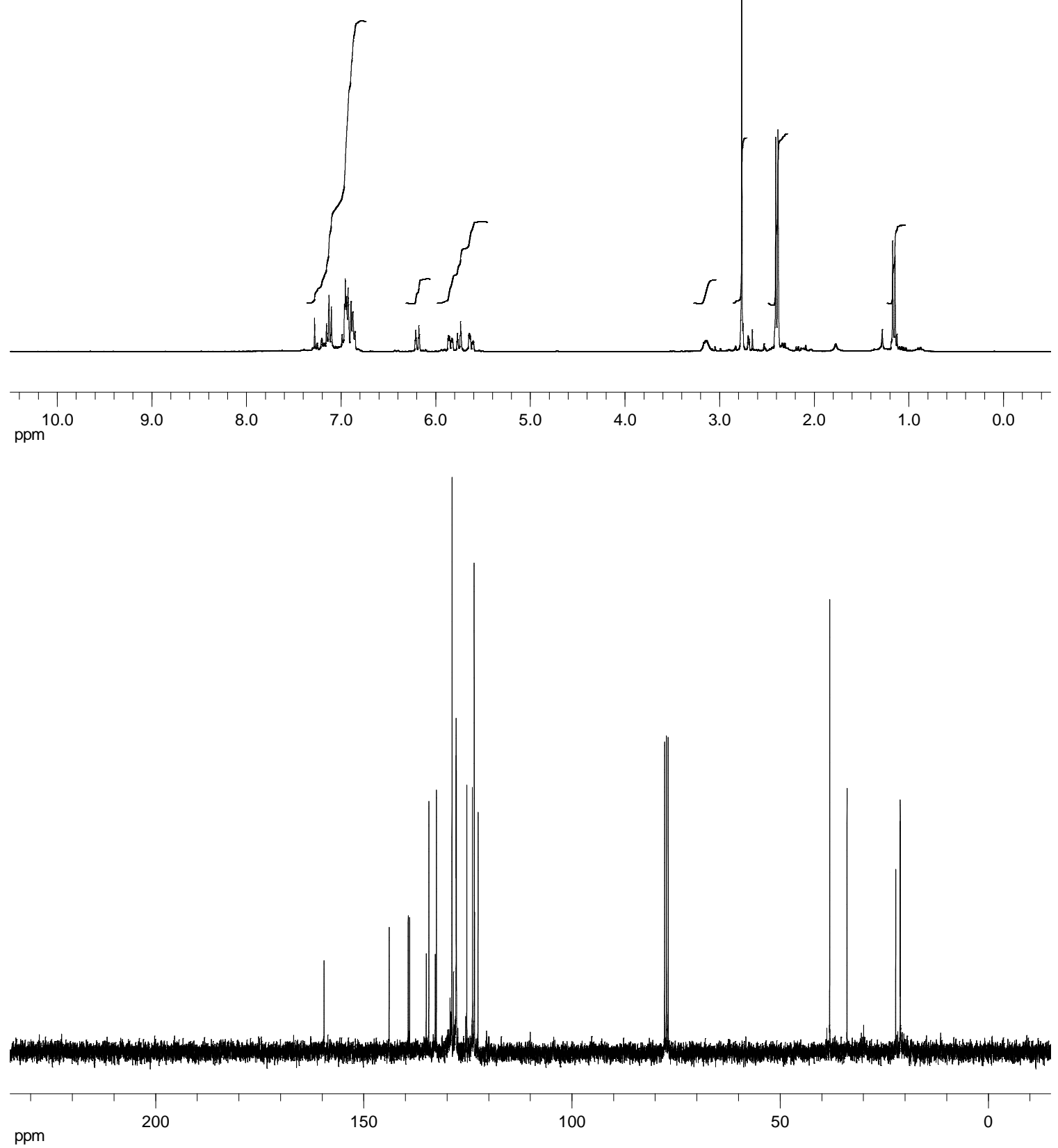
( \pm )-1-((2,6-Dimethylphenyl)benzyl)-3-methyl-1-phenylurea 3a
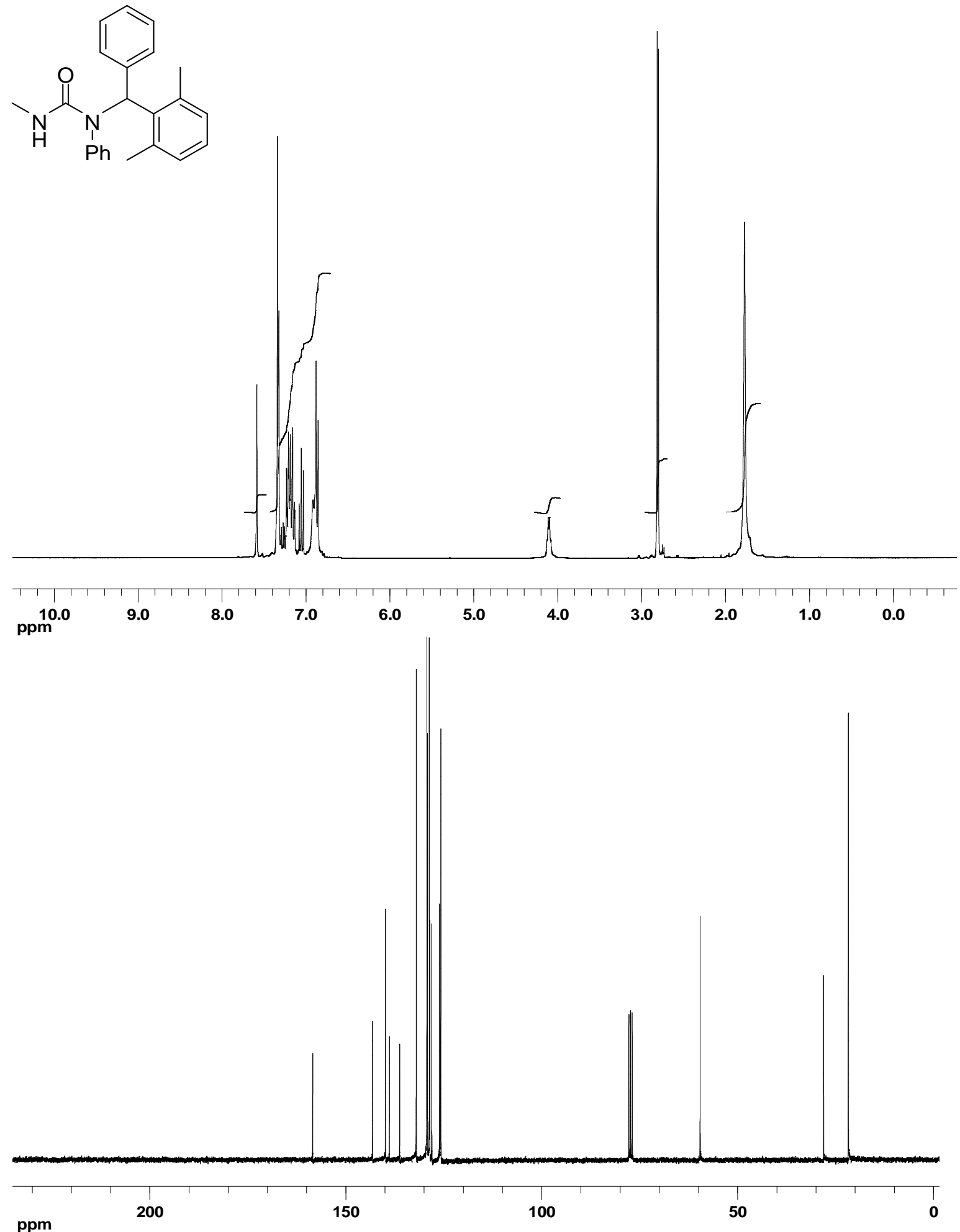
( \pm )-3-Methyl-1-phenyl-1-(phenyl(p-tolyl)methyl)urea 3b<smiles>CNC(=O)N(c1ccccc1)C(c1ccccc1)c1ccccc1</smiles>
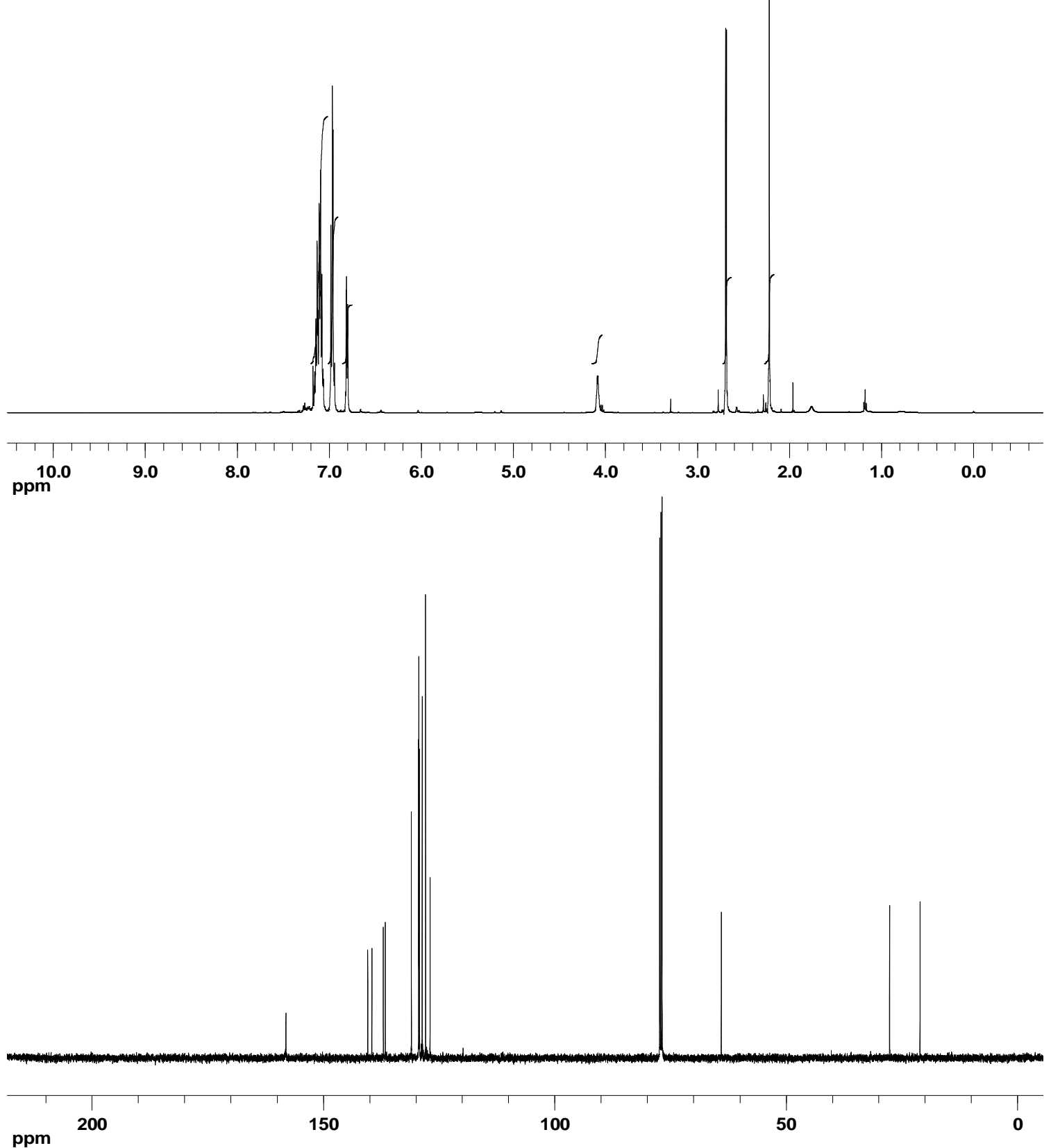


\section{1-Benzhydryl-3-methyl-1-p-tolylurea 3c}<smiles>CNC(=O)N([Ge])C(c1ccccc1)c1ccccc1</smiles>
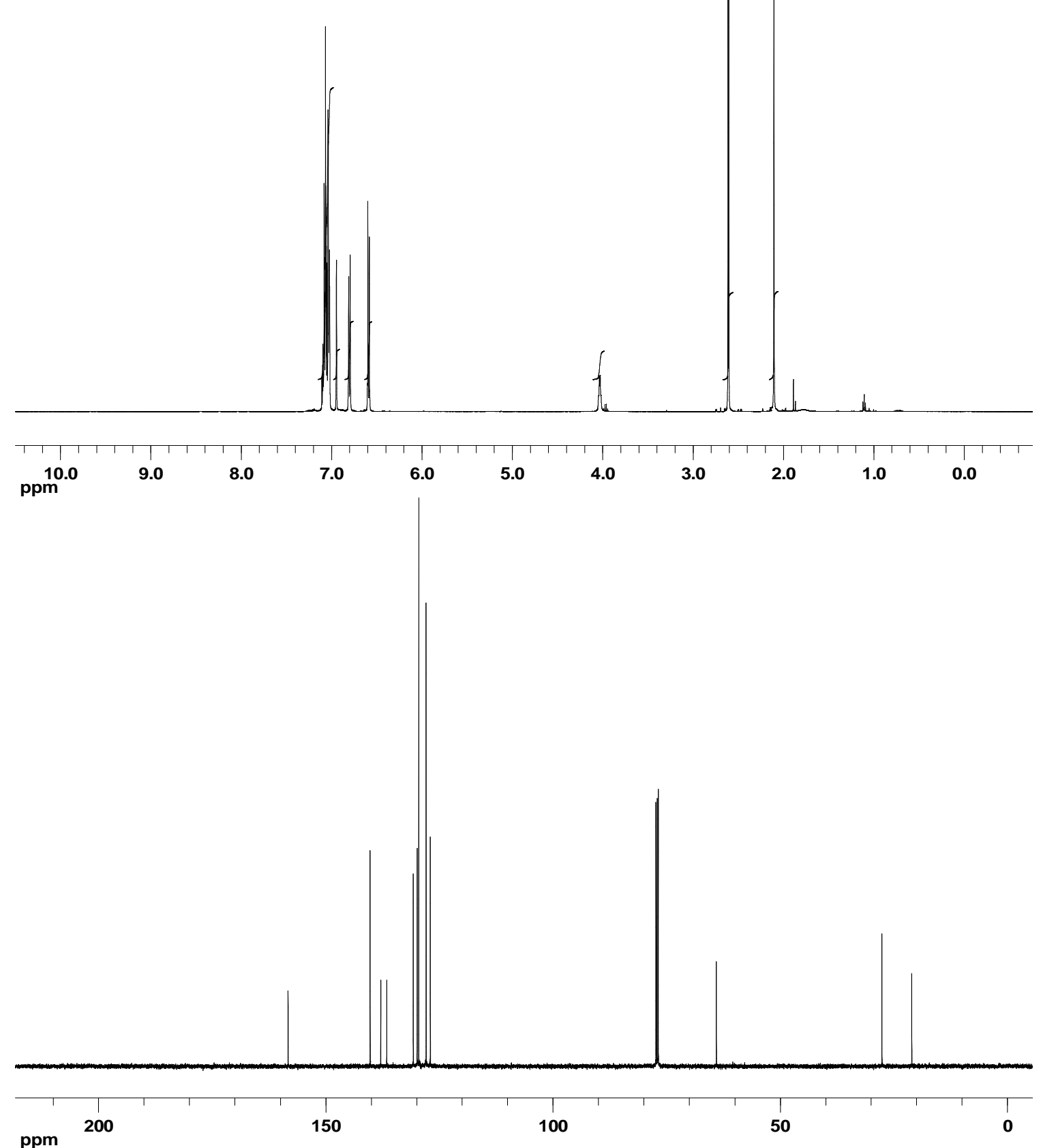

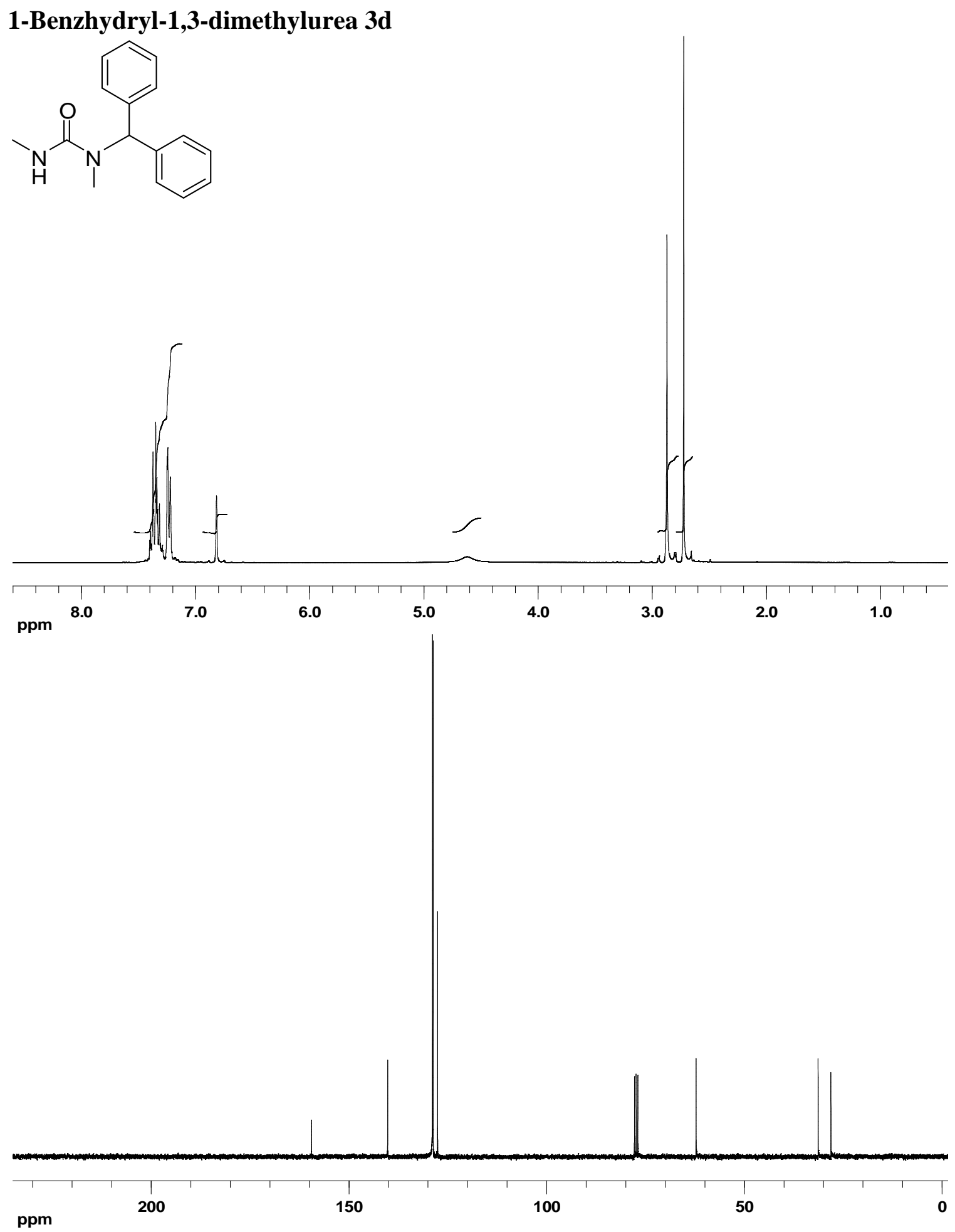
(士)-1,3-Dimethyl-1-(phenyl(o-tolyl)methyl)urea 3e
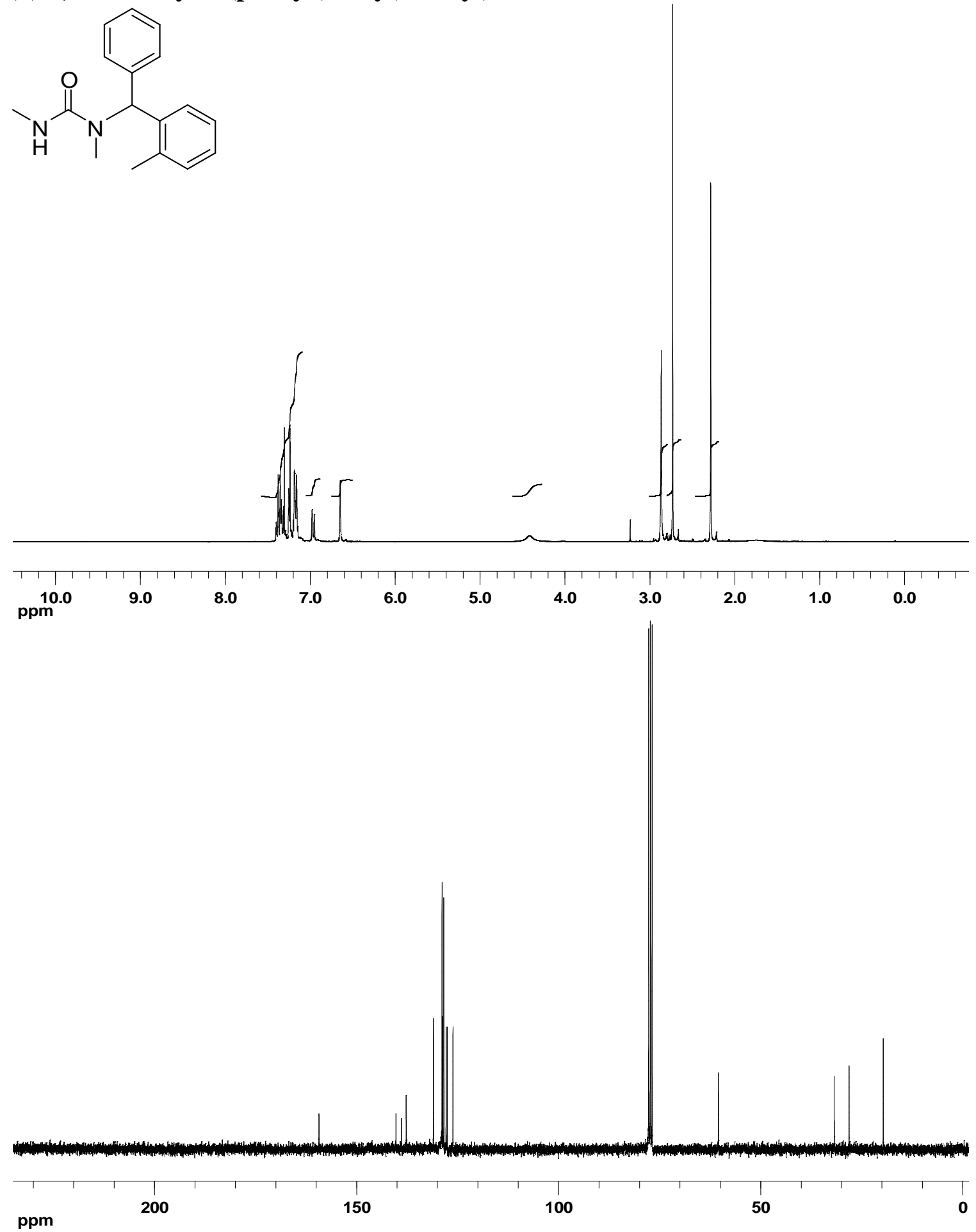
(士)-1-((2-Methoxyphenyl)benzyl)-1,3-dimethylurea 3f
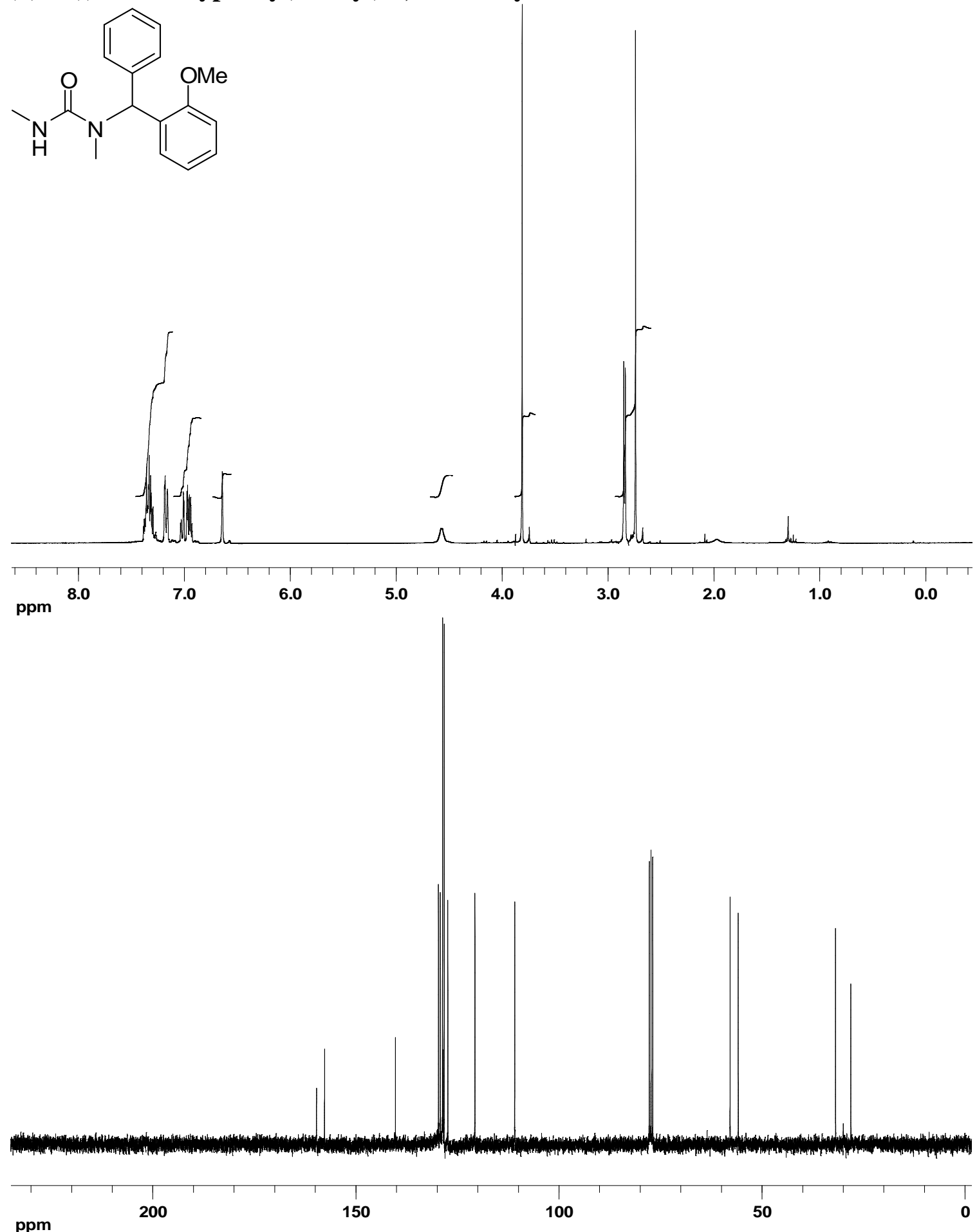
(士)-1-((2,6-Dimethylphenyl)benzyl)-1,3-dimethylurea 3g
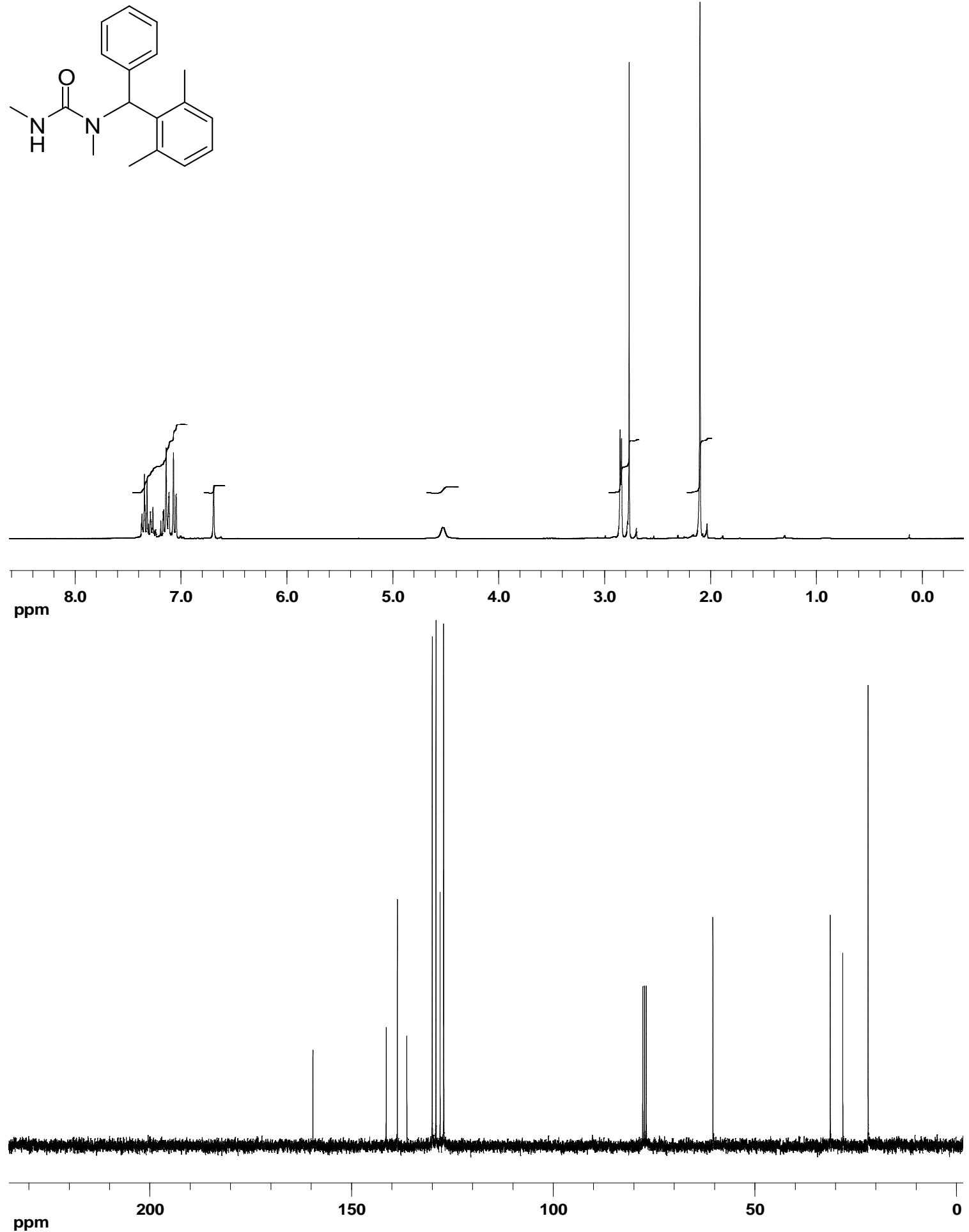
(士)-1-((4-Chlorophenyl)benzyl)-1,3-dimethylurea 3h<smiles>CNC(=O)N(C)C(c1ccccc1)c1ccc(Cl)cc1</smiles>
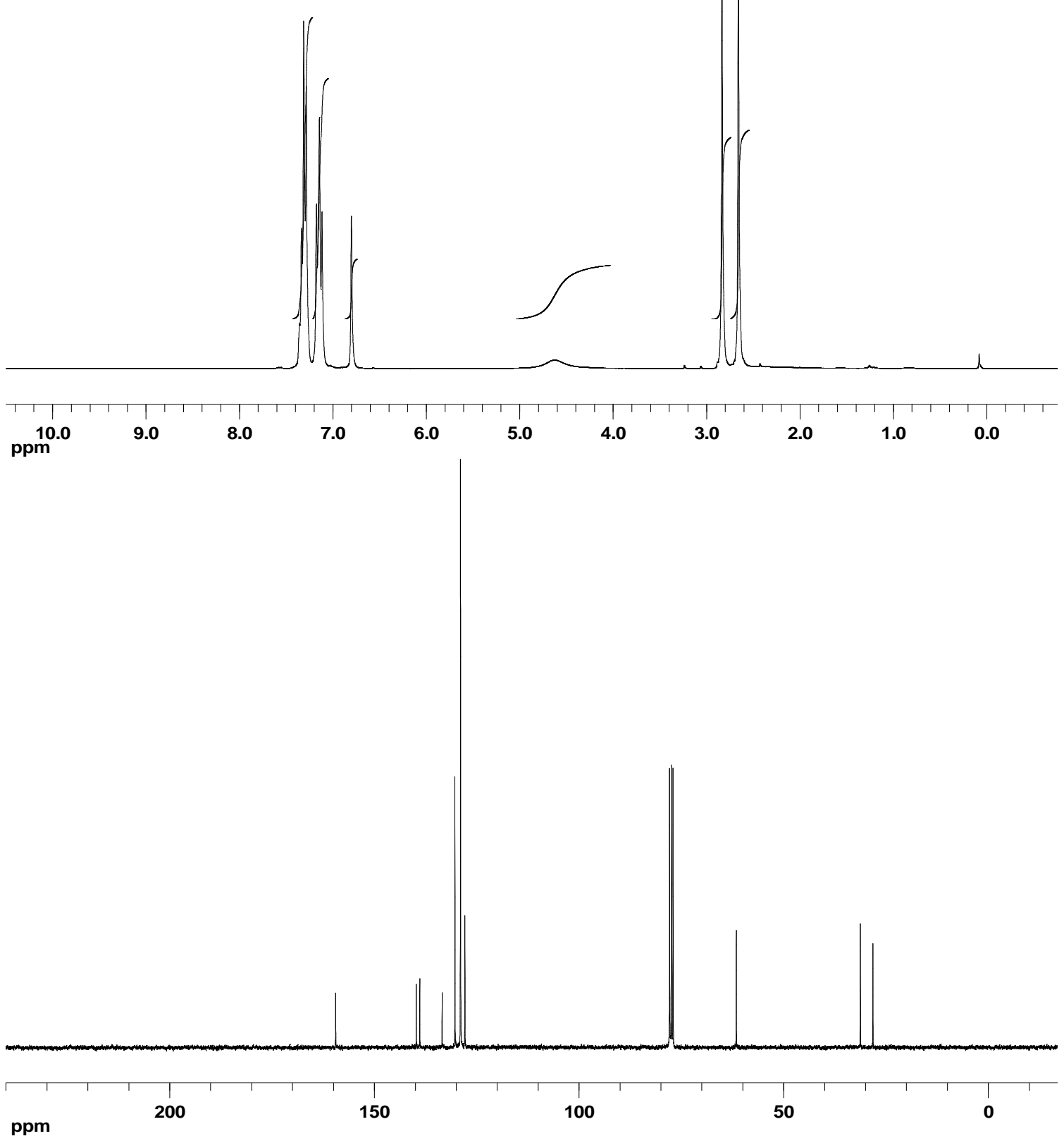
(士)-1-((4-Methoxyphenyl)benzyl)-1,3-dimethylurea 3i<smiles>CNC(=O)N(C)C(c1ccccc1)c1ccc(OC)cc1</smiles>
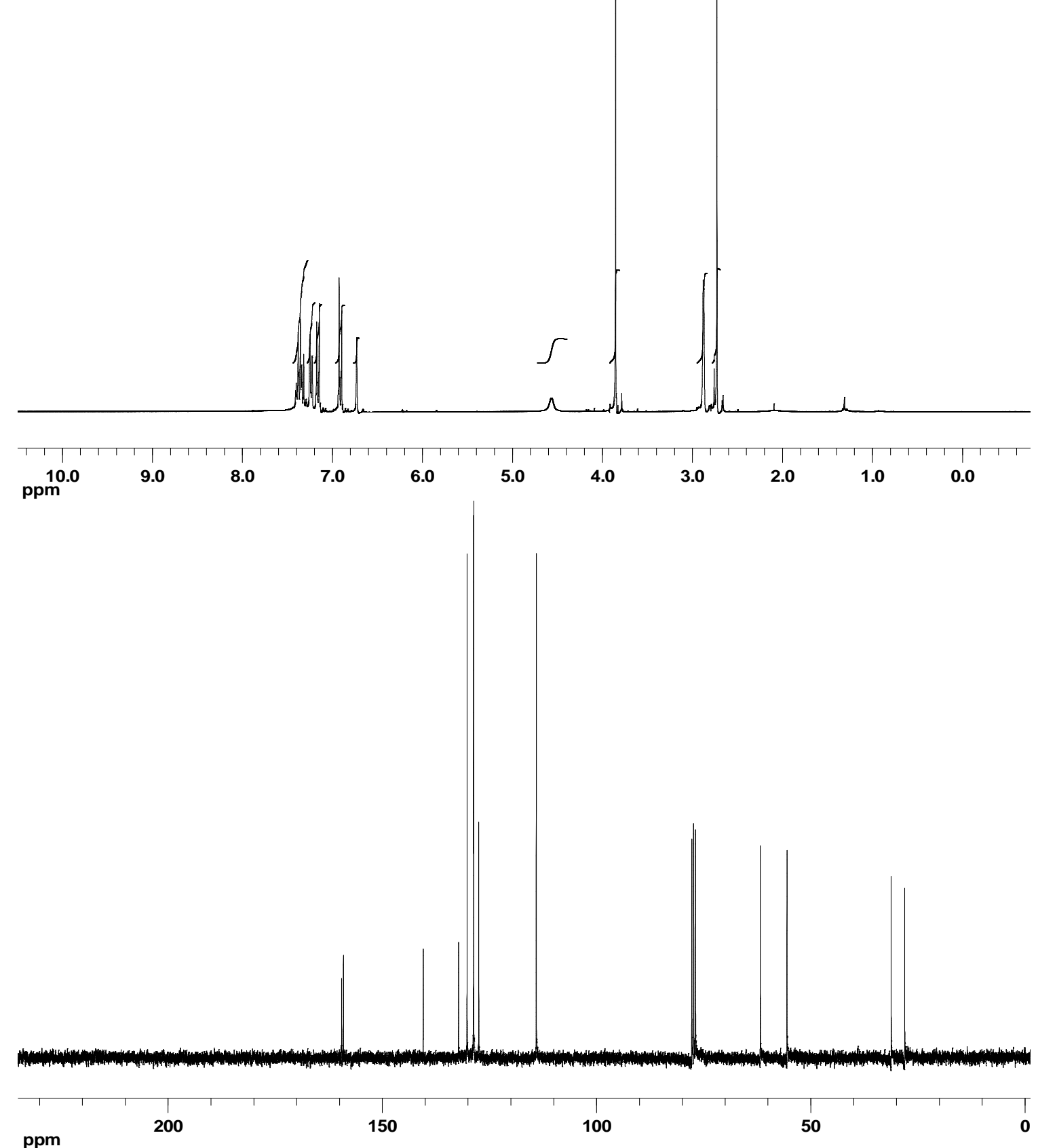
$N$-Methyldiphenylmethanamine $4 \mathrm{~d}$<smiles>CNC(c1ccccc1)c1ccccc1</smiles>
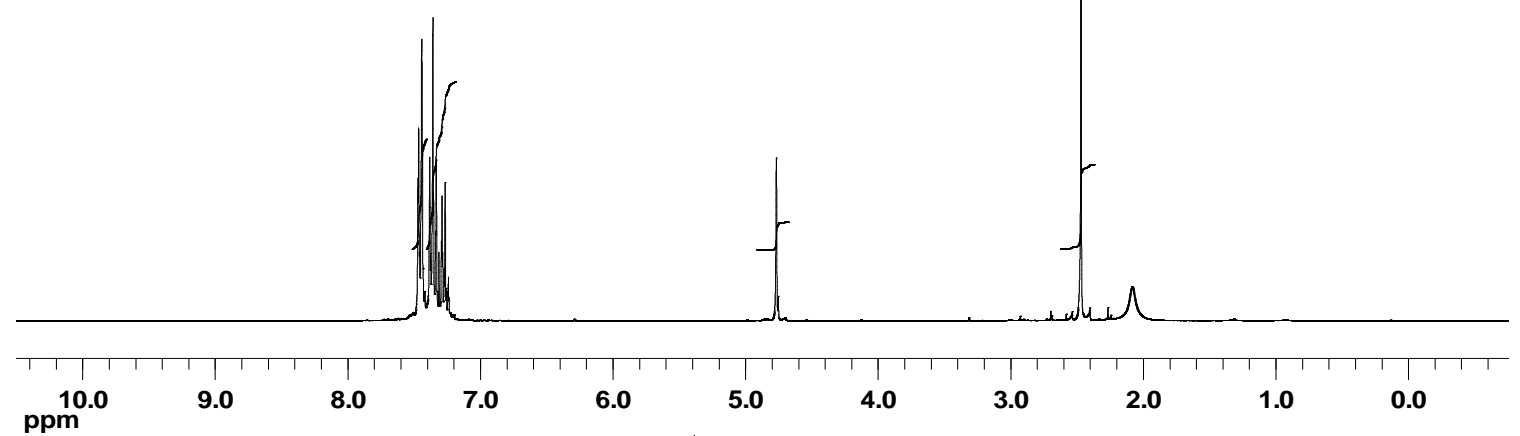

ppm

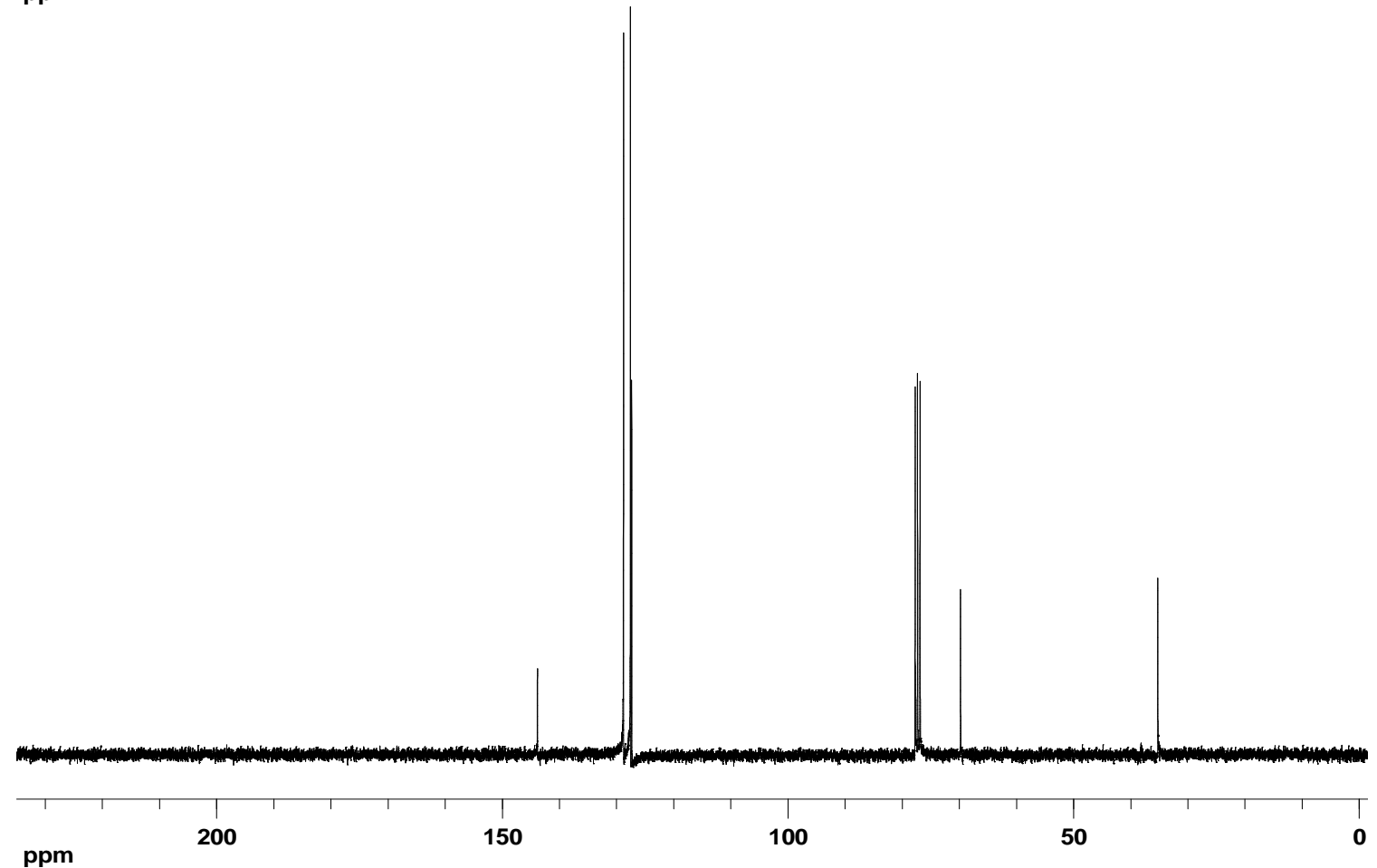

ppm 
( \pm )- $N$-Methyl-1-phenyl-1-o-tolylmethanamine $4 \mathrm{e}$<smiles>CNC(c1ccccc1)c1ccccc1C</smiles>

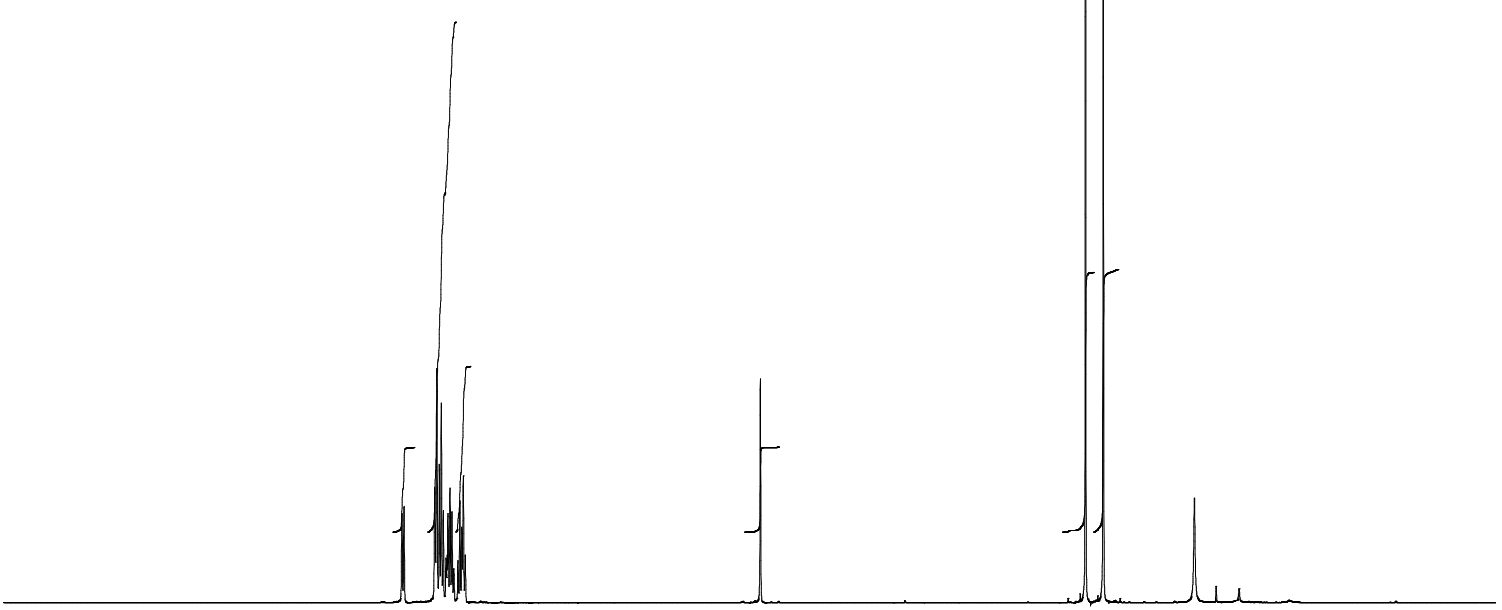

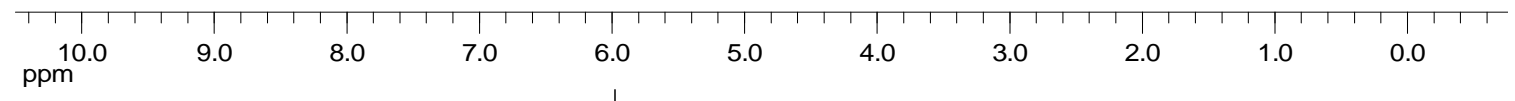

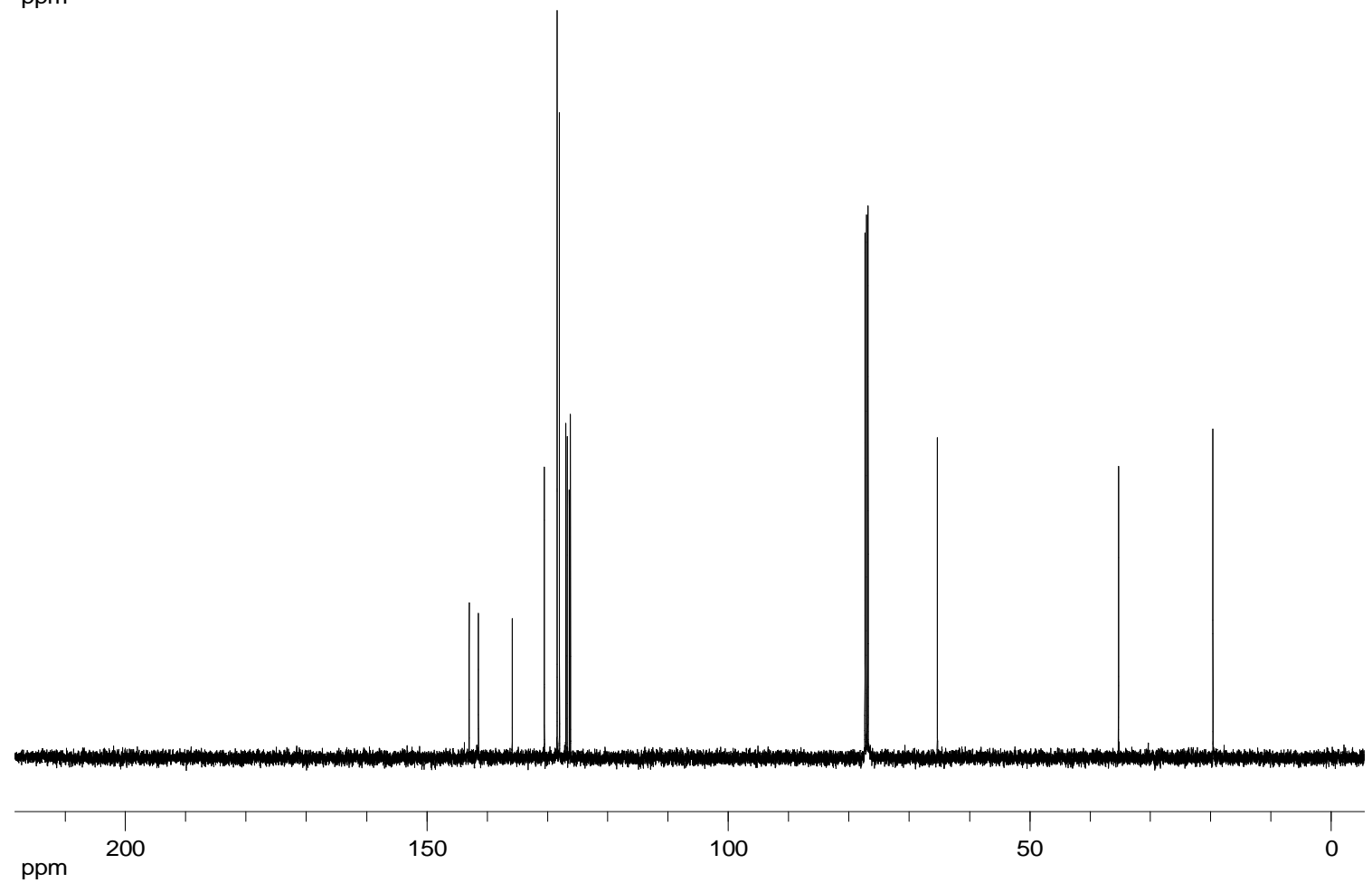


( \pm )-1-(2-Methoxyphenyl)- $N$-methyl-1-phenylmethanamine $4 f$<smiles>CNC(c1ccccc1)c1ccccc1OC</smiles>

1
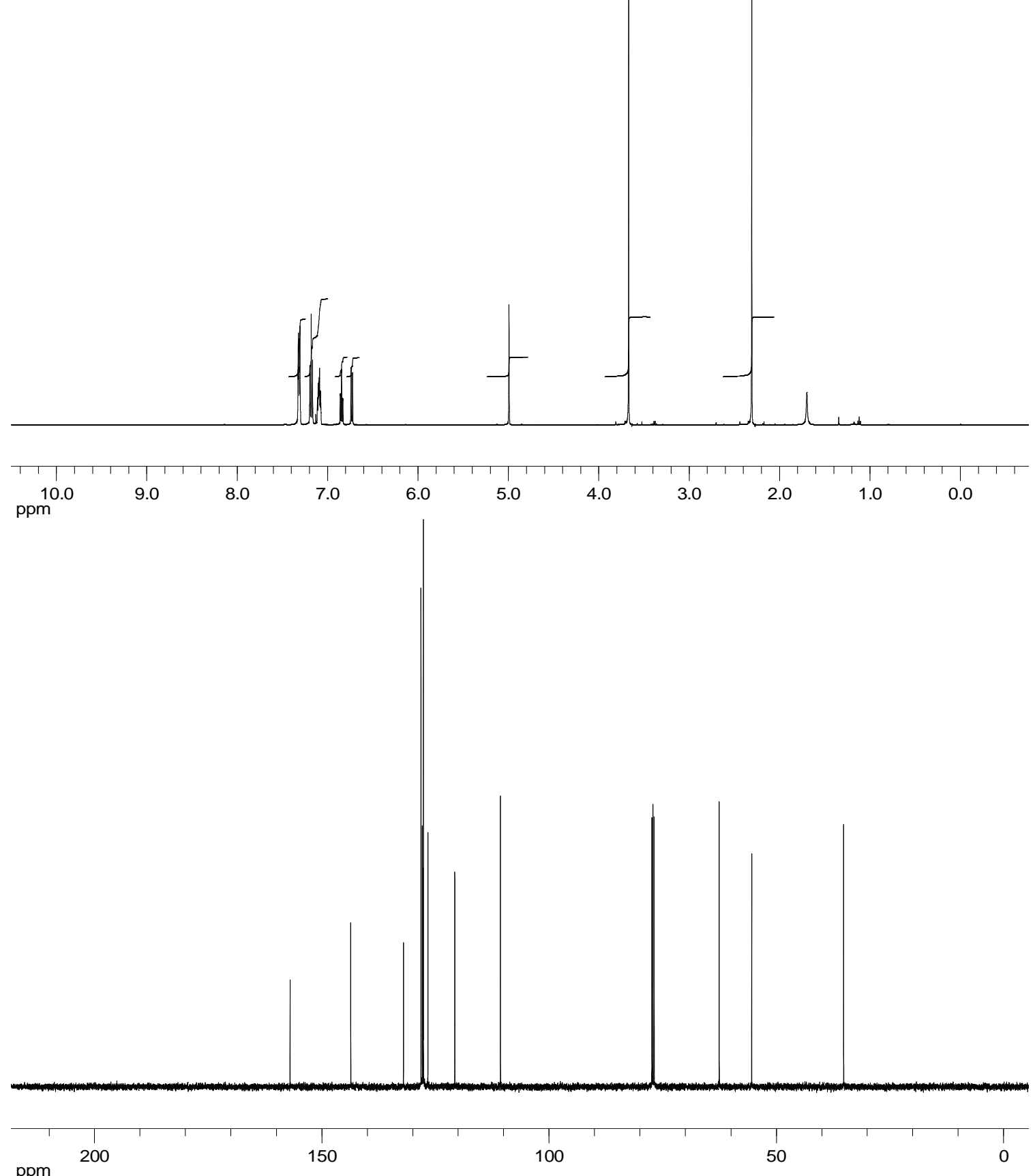
(士)-1-(2,6-Dimethylphenyl)- $N$-methyl-1-phenylmethanamine $4 \mathrm{~g}$<smiles>CNC(c1ccccc1)c1c(C)cccc1C</smiles> 
(士)-1,3-Dimethyl-1-phenyl-3-(1-phenylethyl)urea 5a
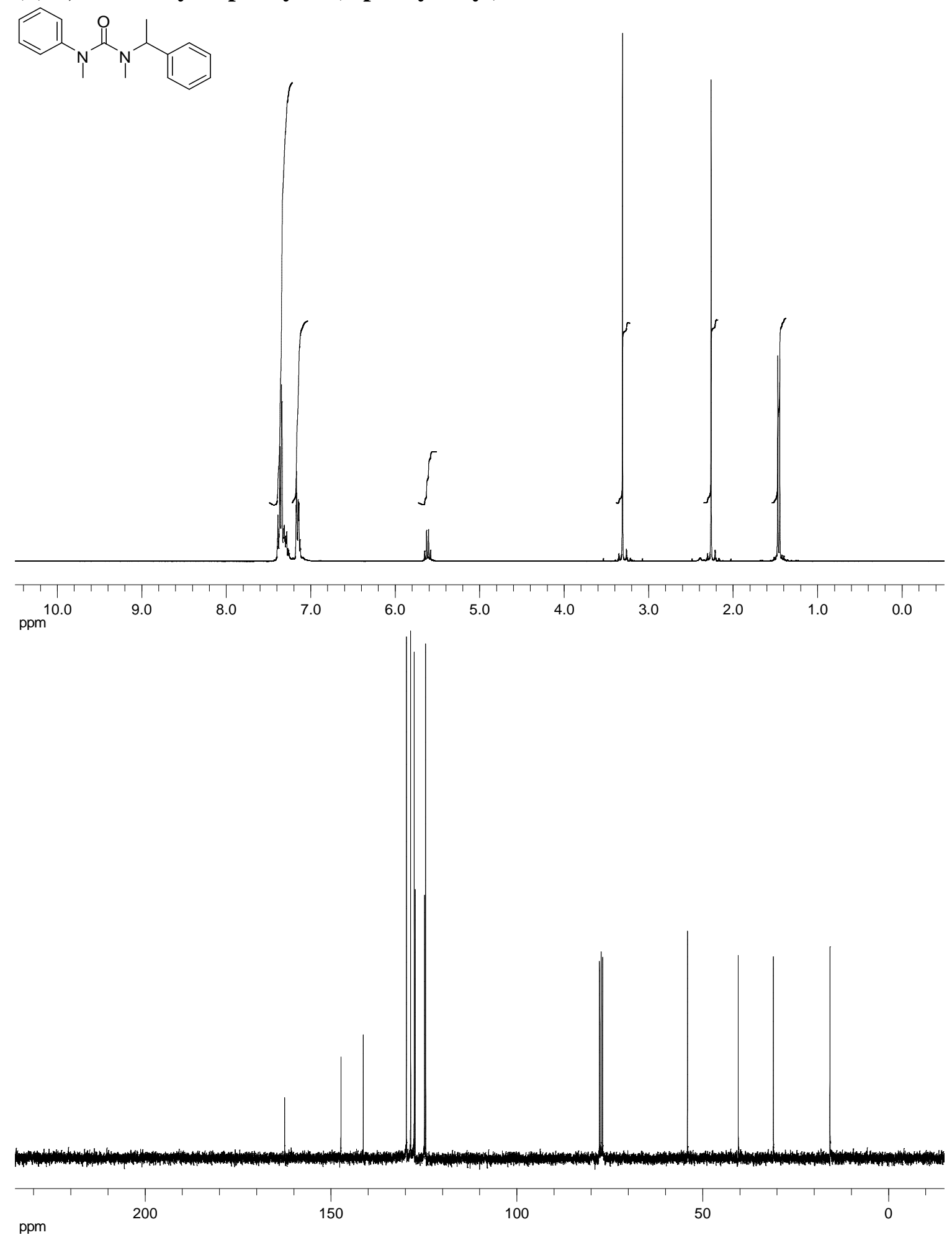

24 
1-(4-Methoxyphenyl)-1,3-dimethyl-3-((R)-1-phenylethyl)urea 5b
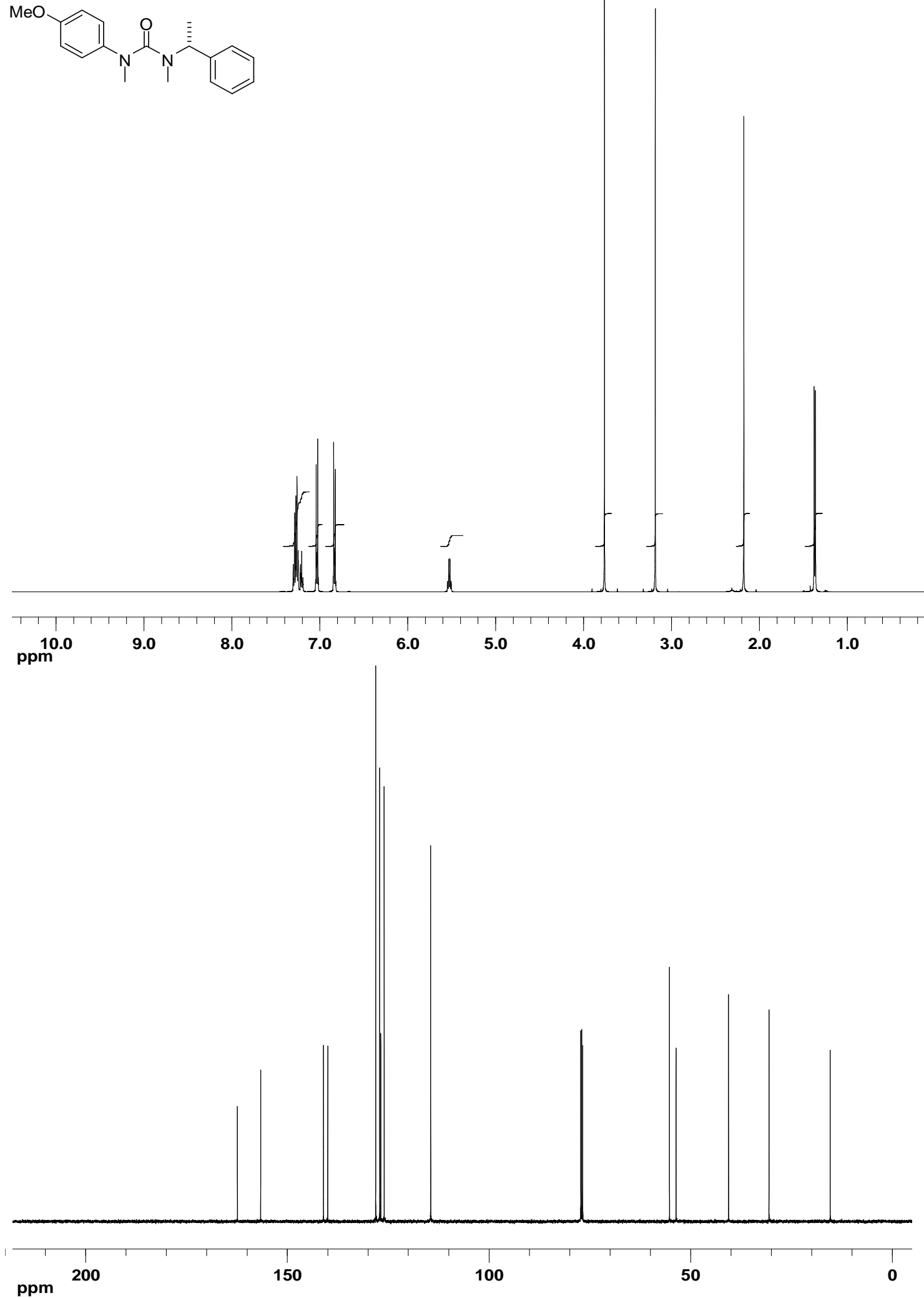
(士)-1-(2-Methoxyphenyl)-1,3-dimethyl-3-(1-phenylethyl)urea 5c
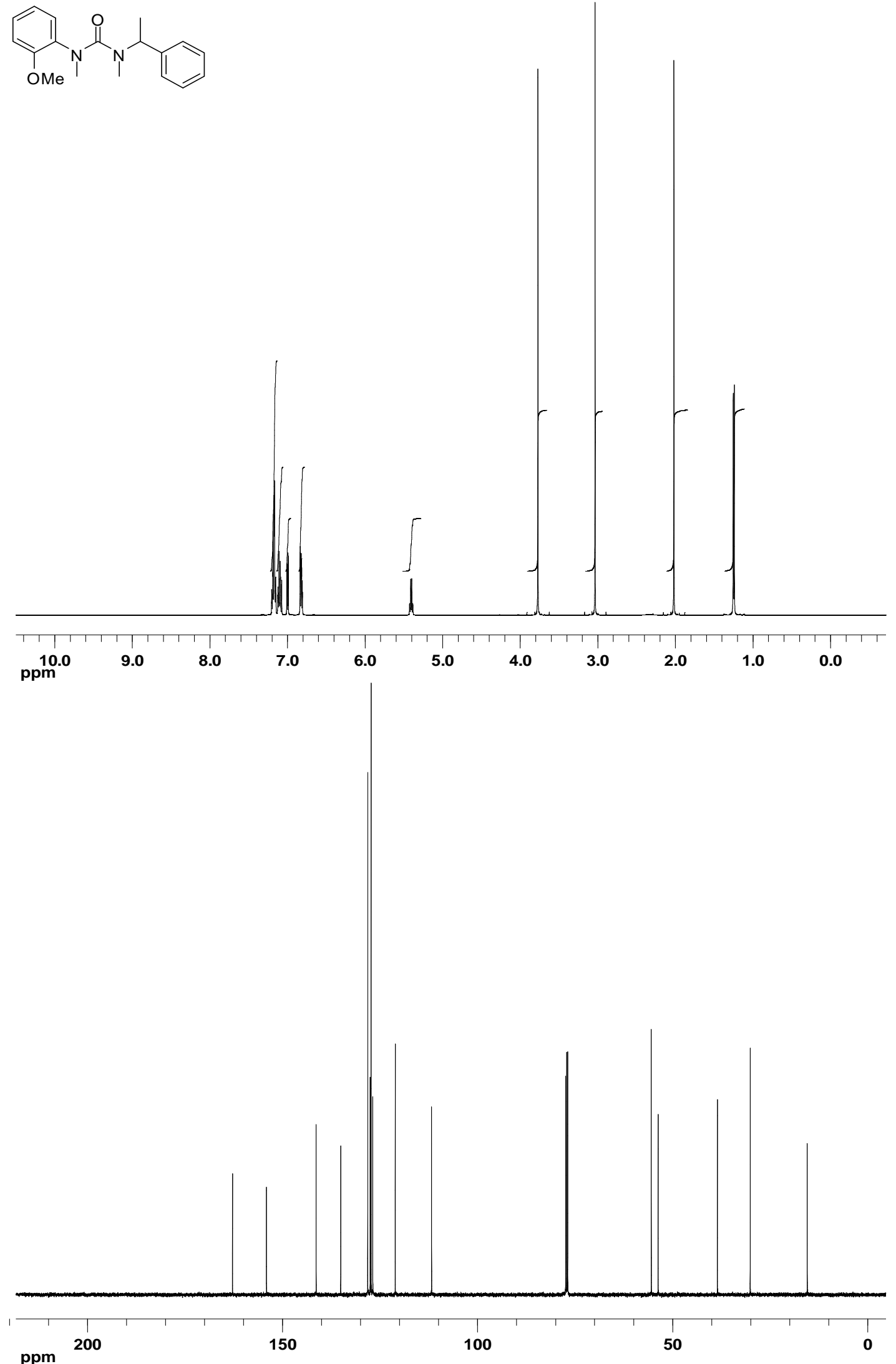
1-(3,4-Dimethoxyphenyl)-1,3-dimethyl-3-((S)-1-phenylethyl)urea 5d
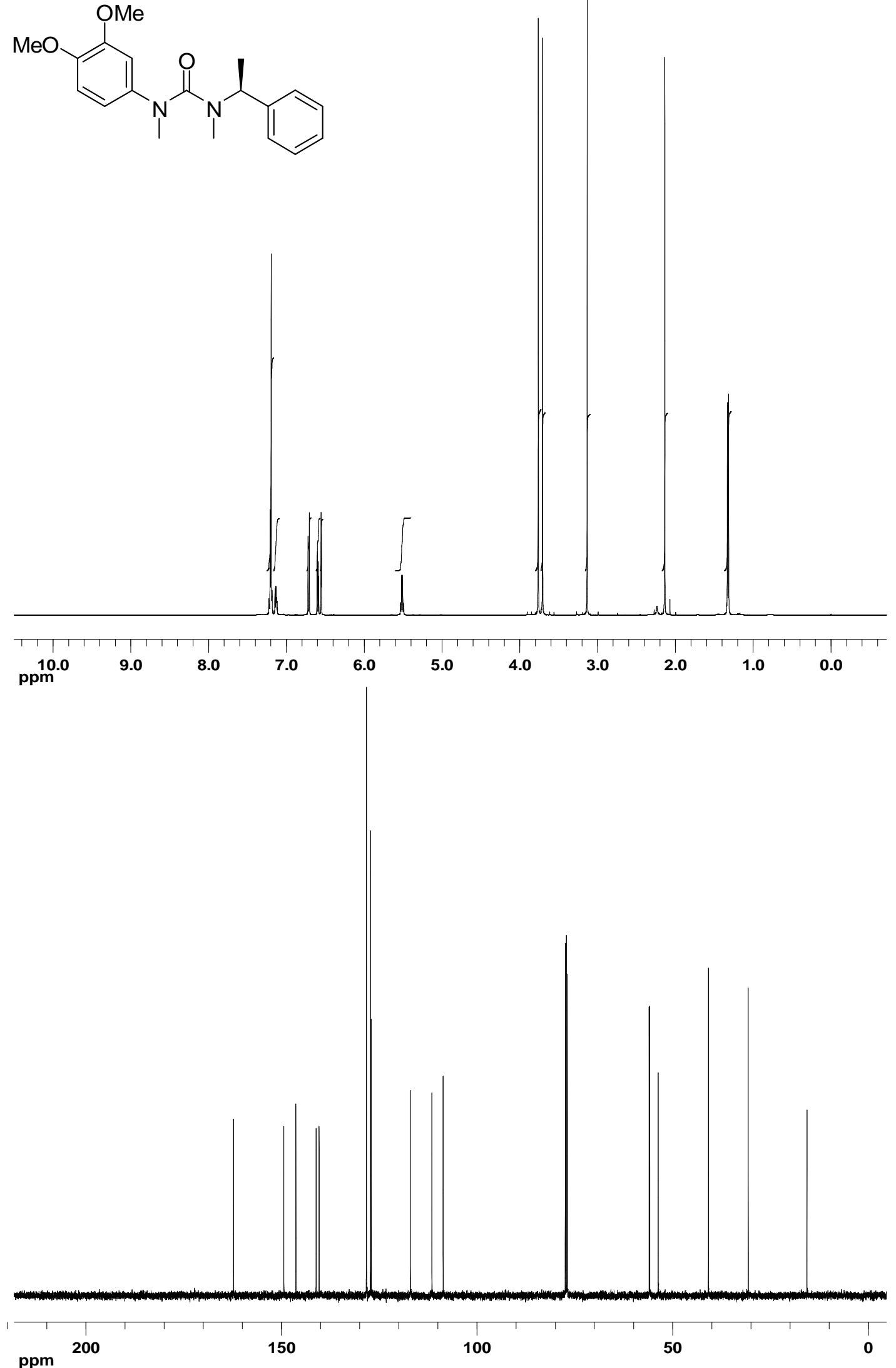
(士)-1-(2-Fluorophenyl)-1,3-dimethyl-3-(1-phenylethyl)urea 5e
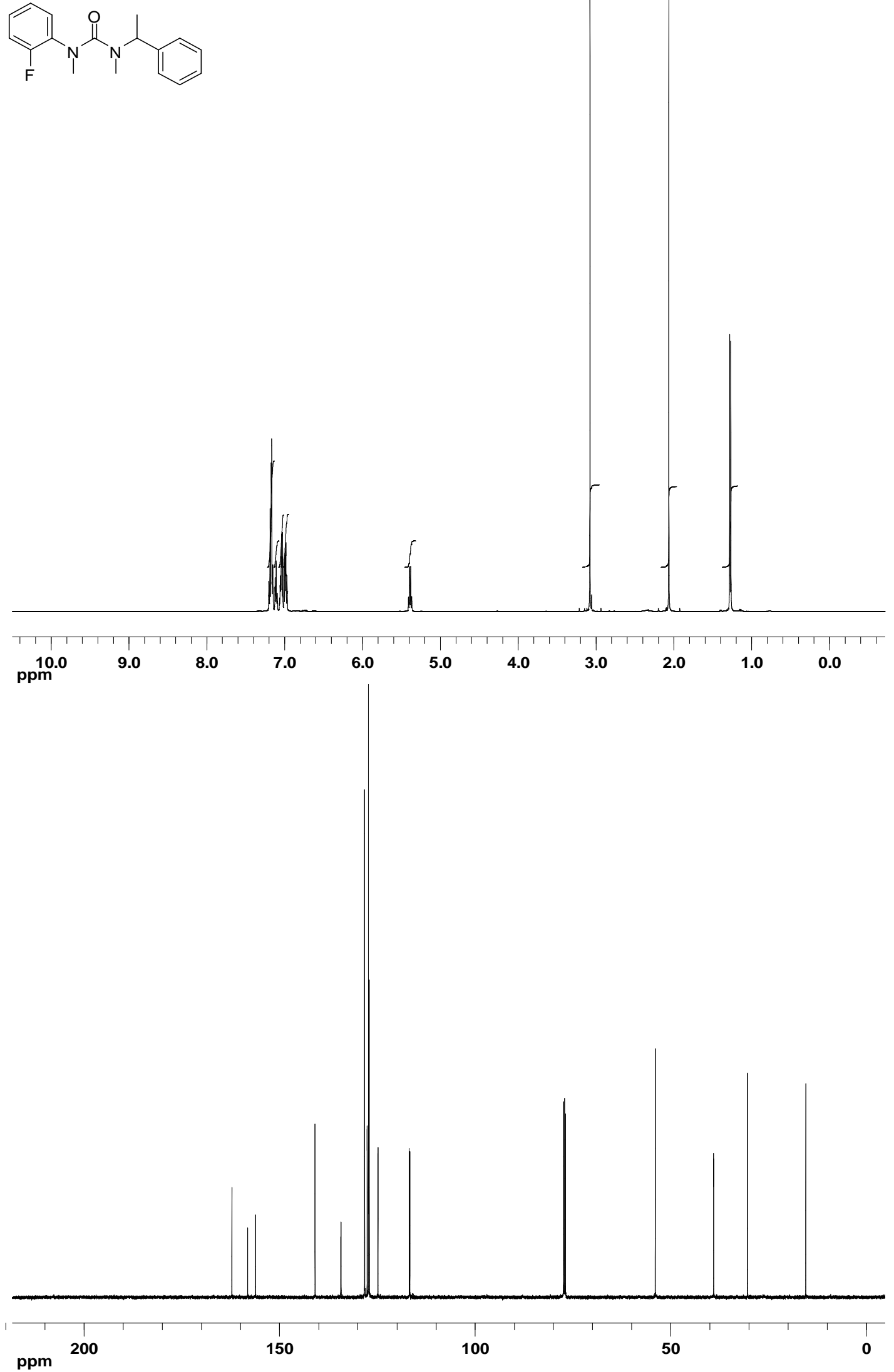


\section{( \pm )-1-(3-Fluorophenyl)-1,3-dimethyl-3-(1-phenylethyl)urea $5 f$}
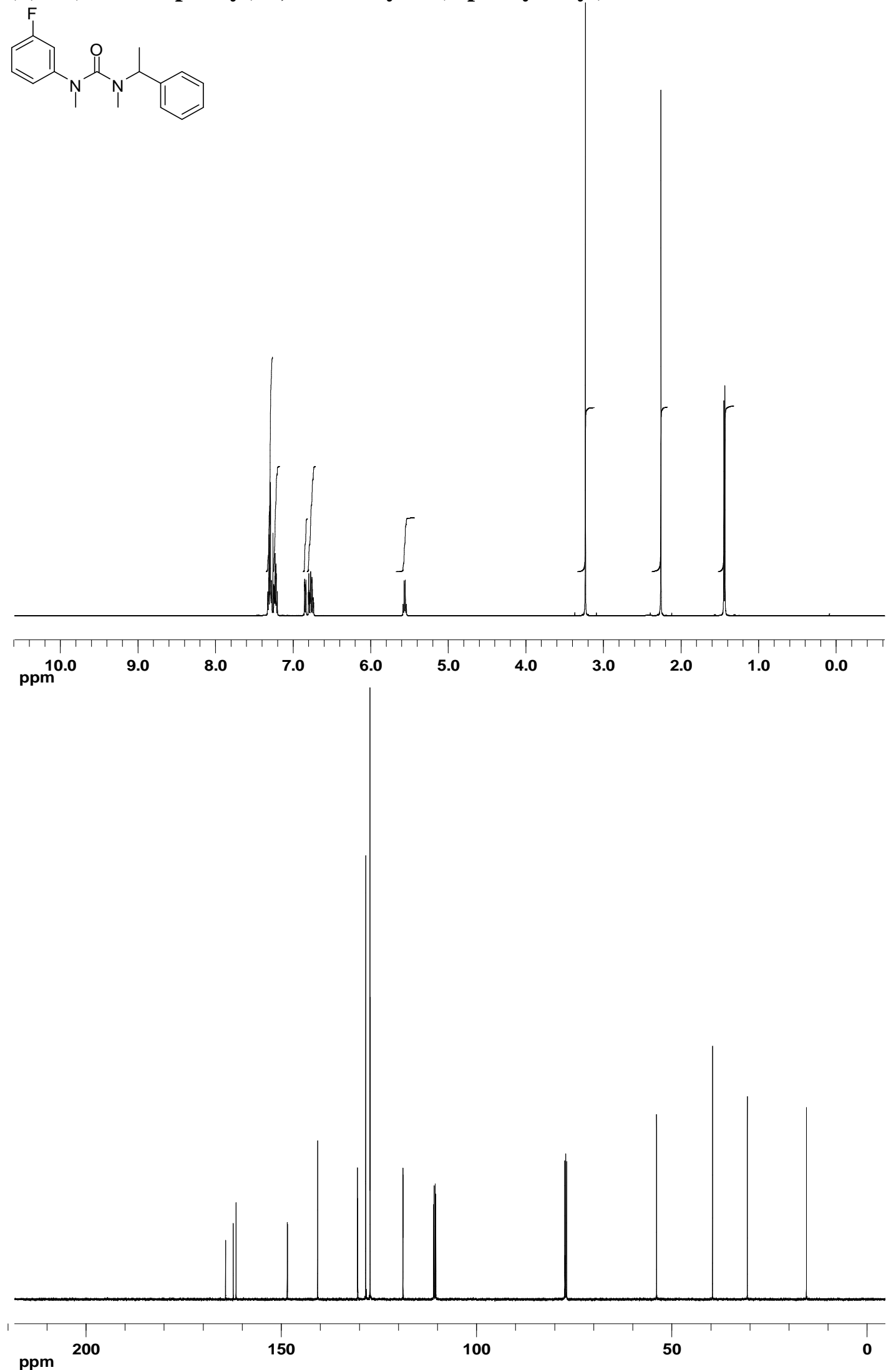
1-(4-Fluorophenyl)-1,3-dimethyl-3-((S)-1-phenylethyl)urea 5g
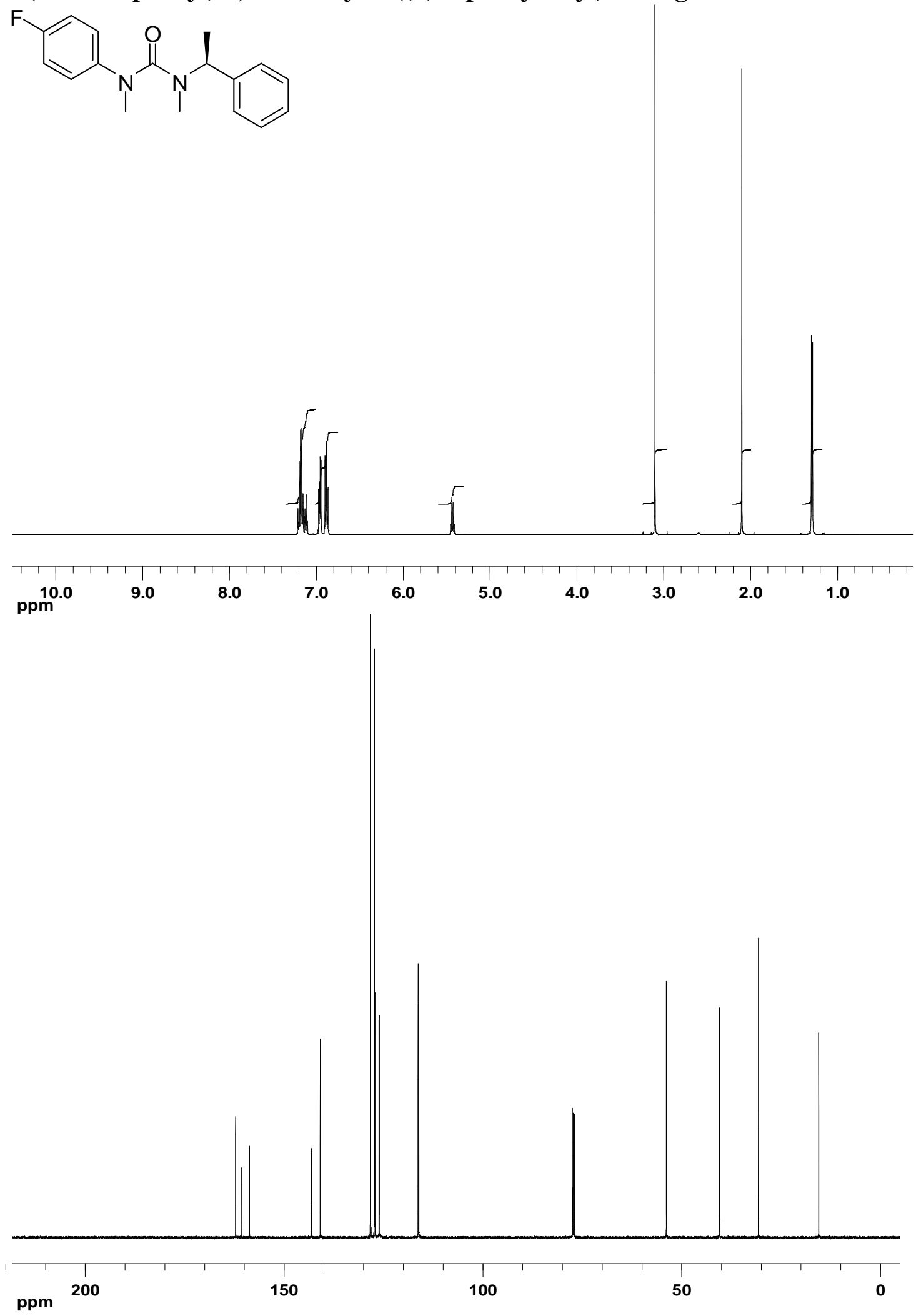
1-(4-Chlorophenyl)-1,3-dimethyl-3-((S)-1-phenylethyl)urea 5h
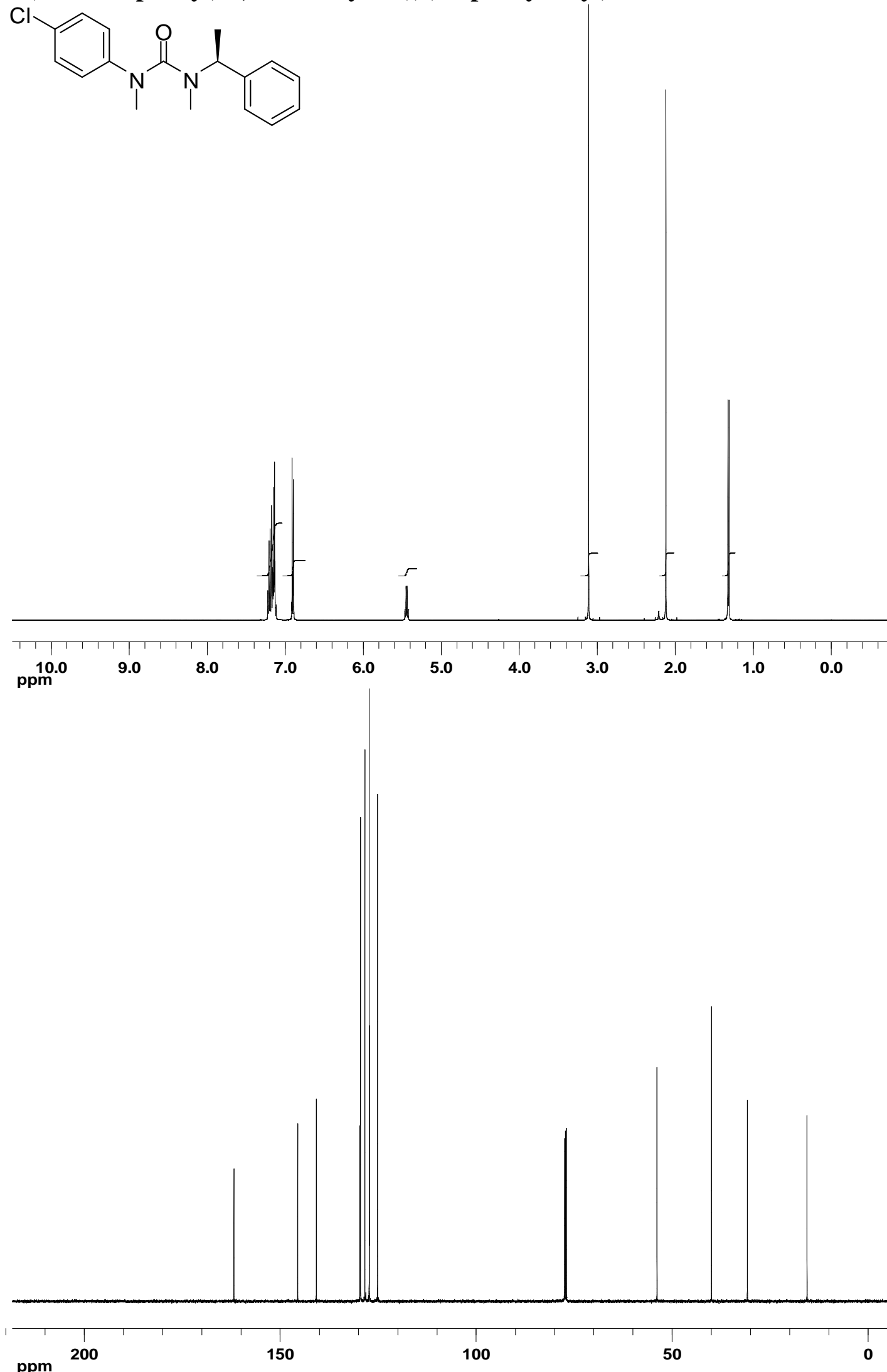


\section{1-(3-Chloro-4-fluorophenyl)-1,3-dimethyl-3-((S)-1-phenylethyl)urea 5i}
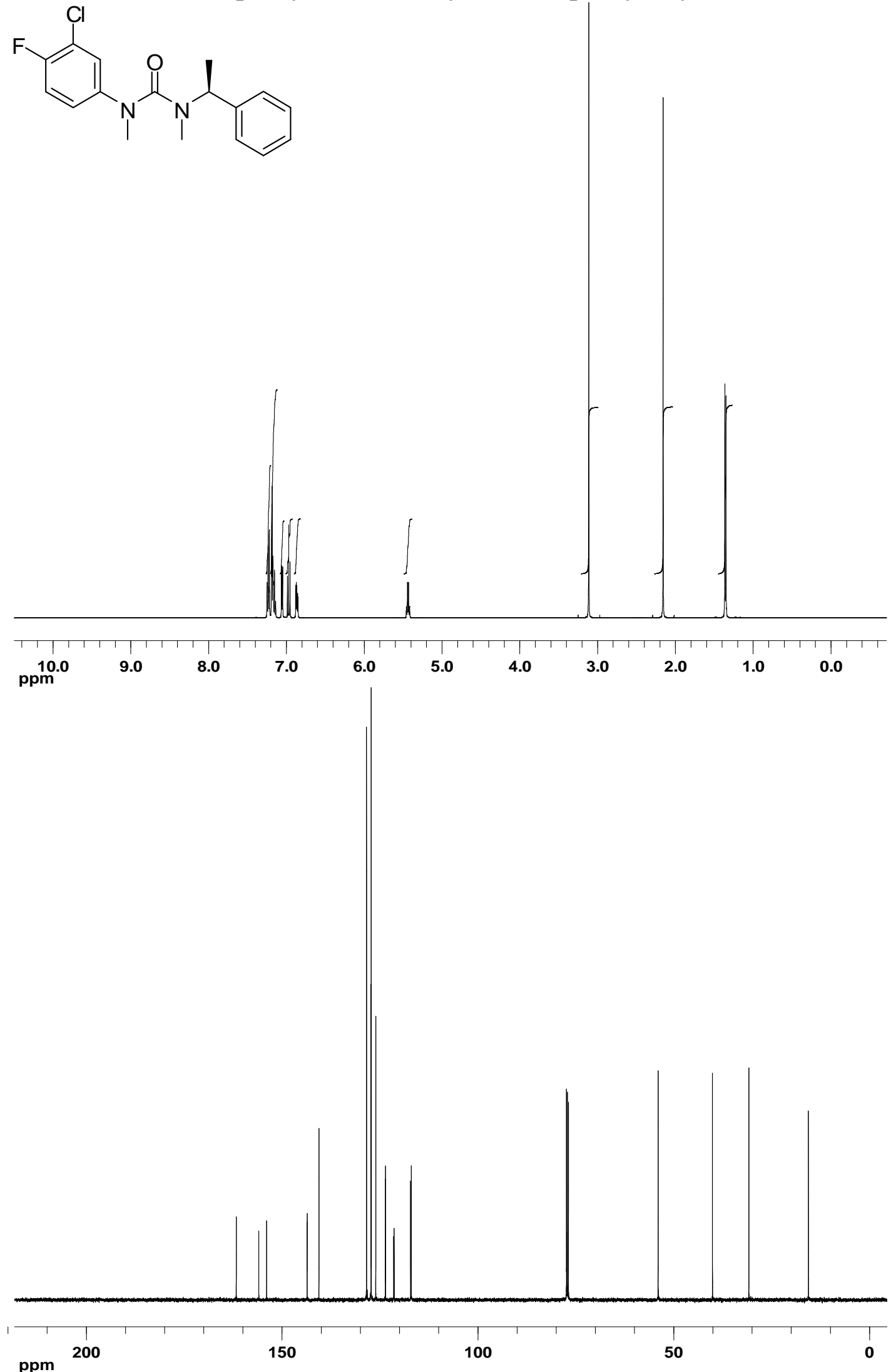
1,3-Dimethyl-1-(naphthalen-1-yl)-3-((S)-1-phenylethyl)urea 5j<smiles>CC(c1ccccc1)N(C)C(=O)N(C)c1cccc2ccccc12</smiles>
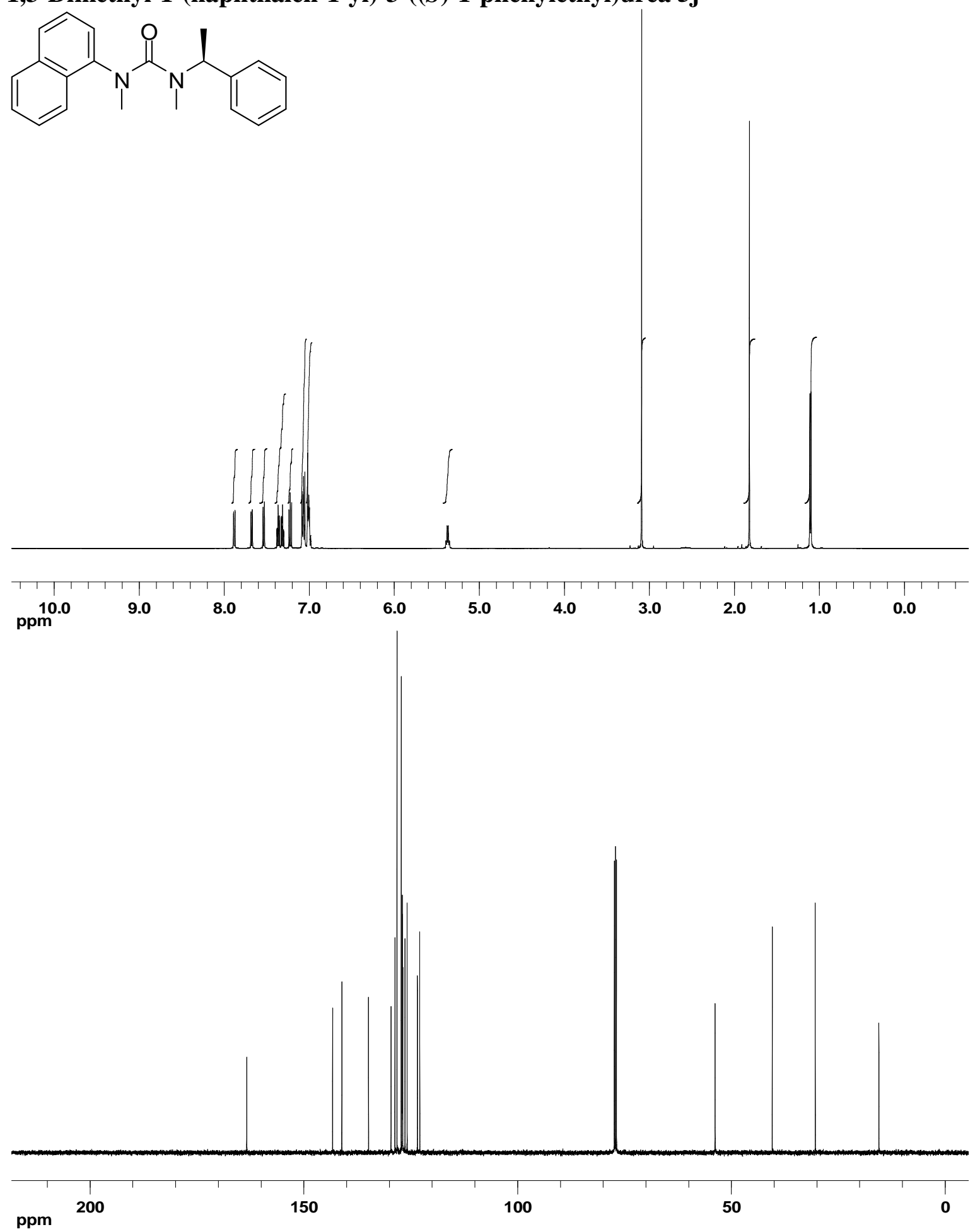
1,3-Dimethyl-1-(naphthalen-3-yl)-3-((S)-1-phenylethyl)urea 5k

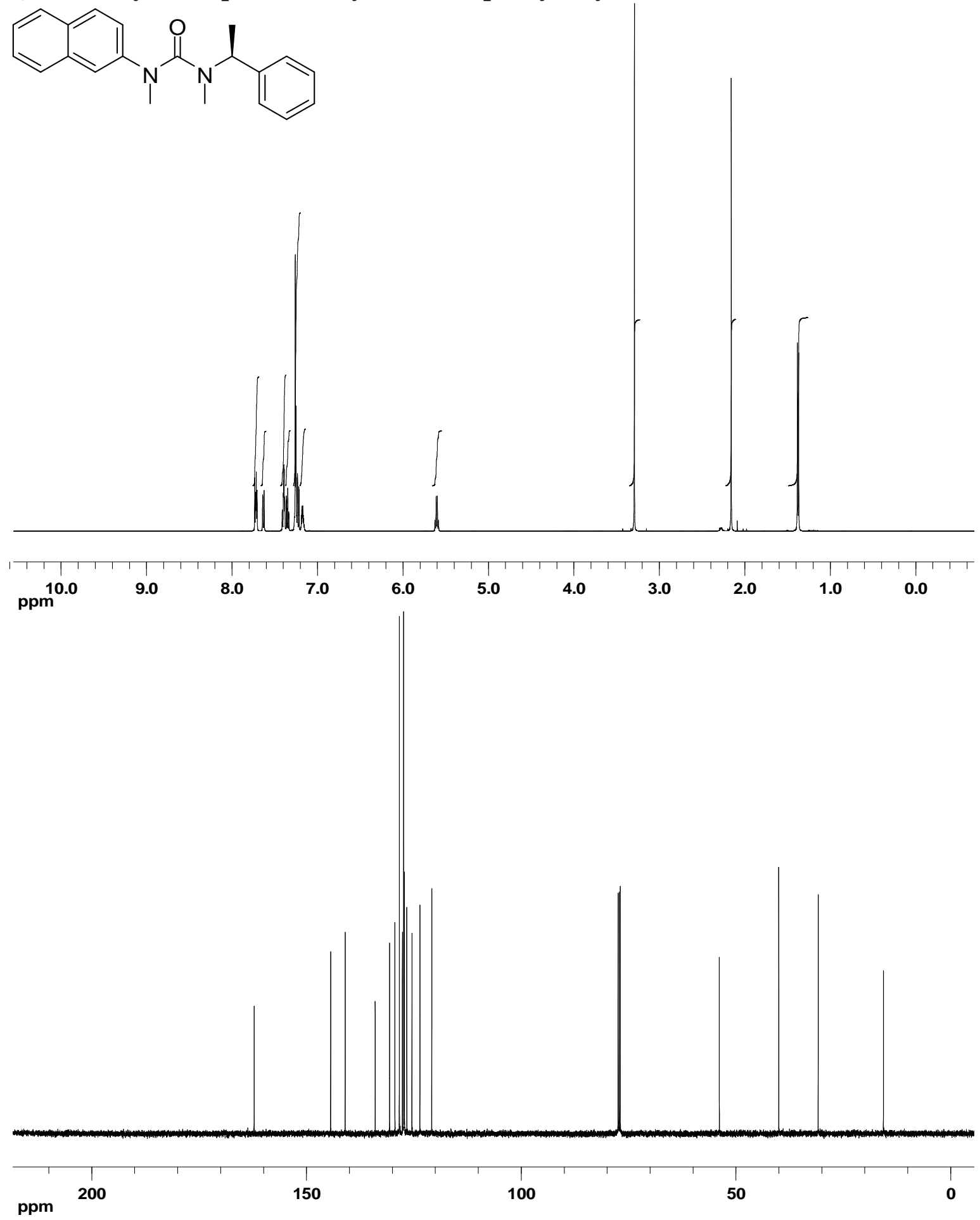


1-((R)-1-(4-Methoxyphenyl)ethyl)-1,3-dimethyl-3-phenylurea 5l
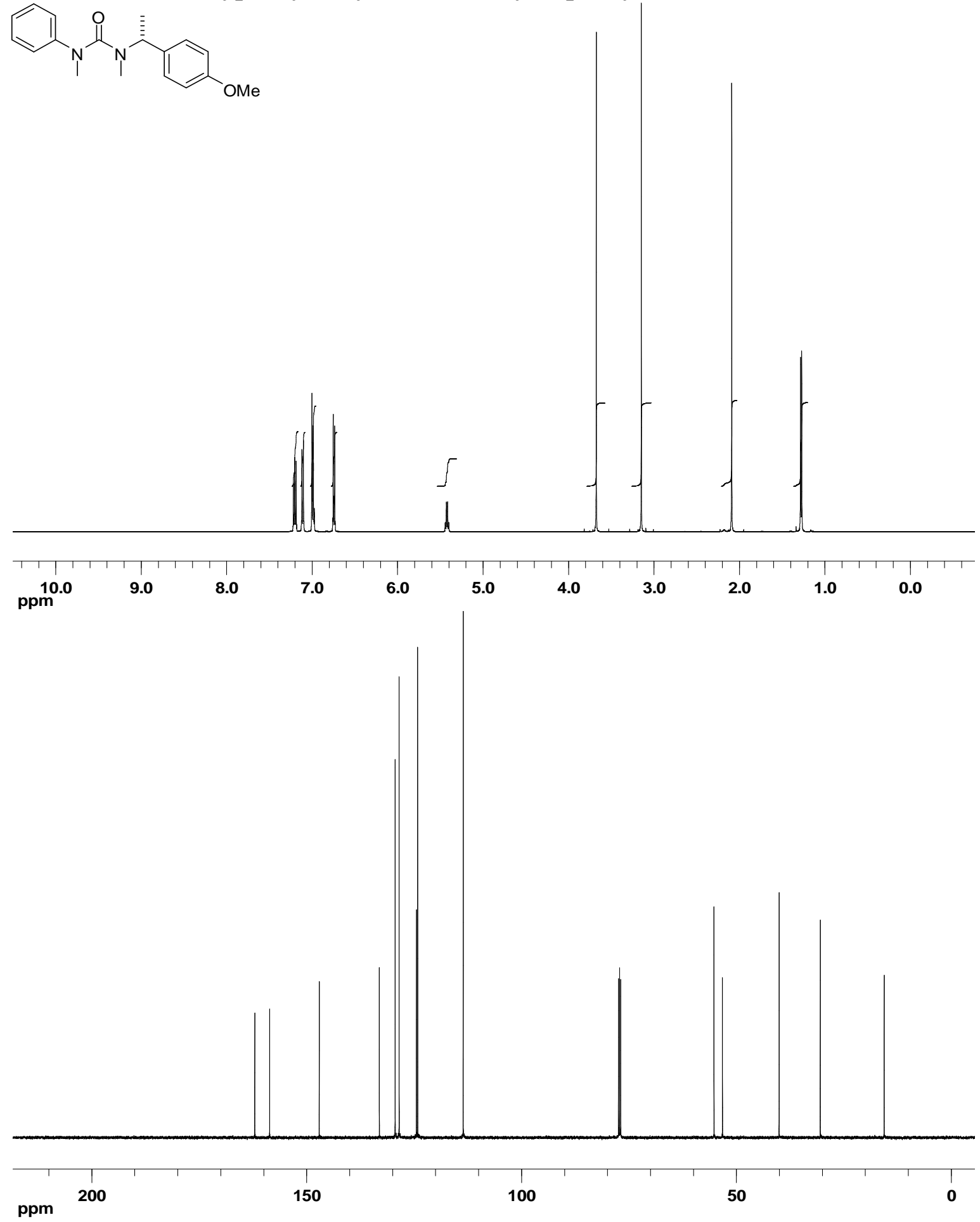
1-((S)-1-(4-Methoxyphenyl)ethyl)-1,3-dimethyl-3-o-tolylurea 5m<smiles>COc1ccc([C@H](C)N(C)C(=O)N(C)c2ccccc2C)cc1</smiles>
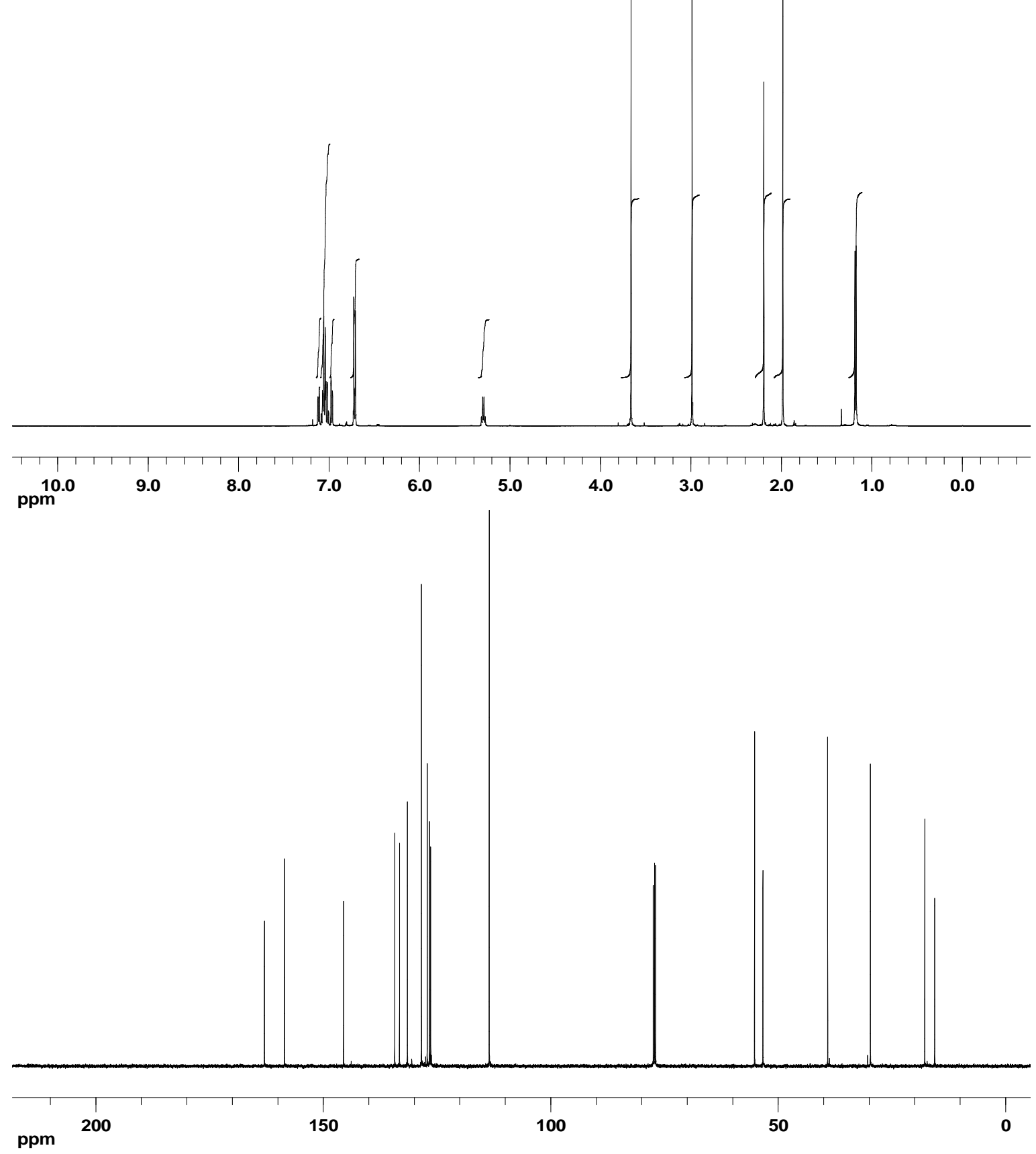


\section{1-((S)-1-(4-Methoxyphenyl)ethyl)-1,3-dimethyl-3-p-tolylurea 5n}<smiles>COc1ccc([C@H](C)N(C)C(=O)N(C)c2ccc(C)cc2)cc1</smiles>
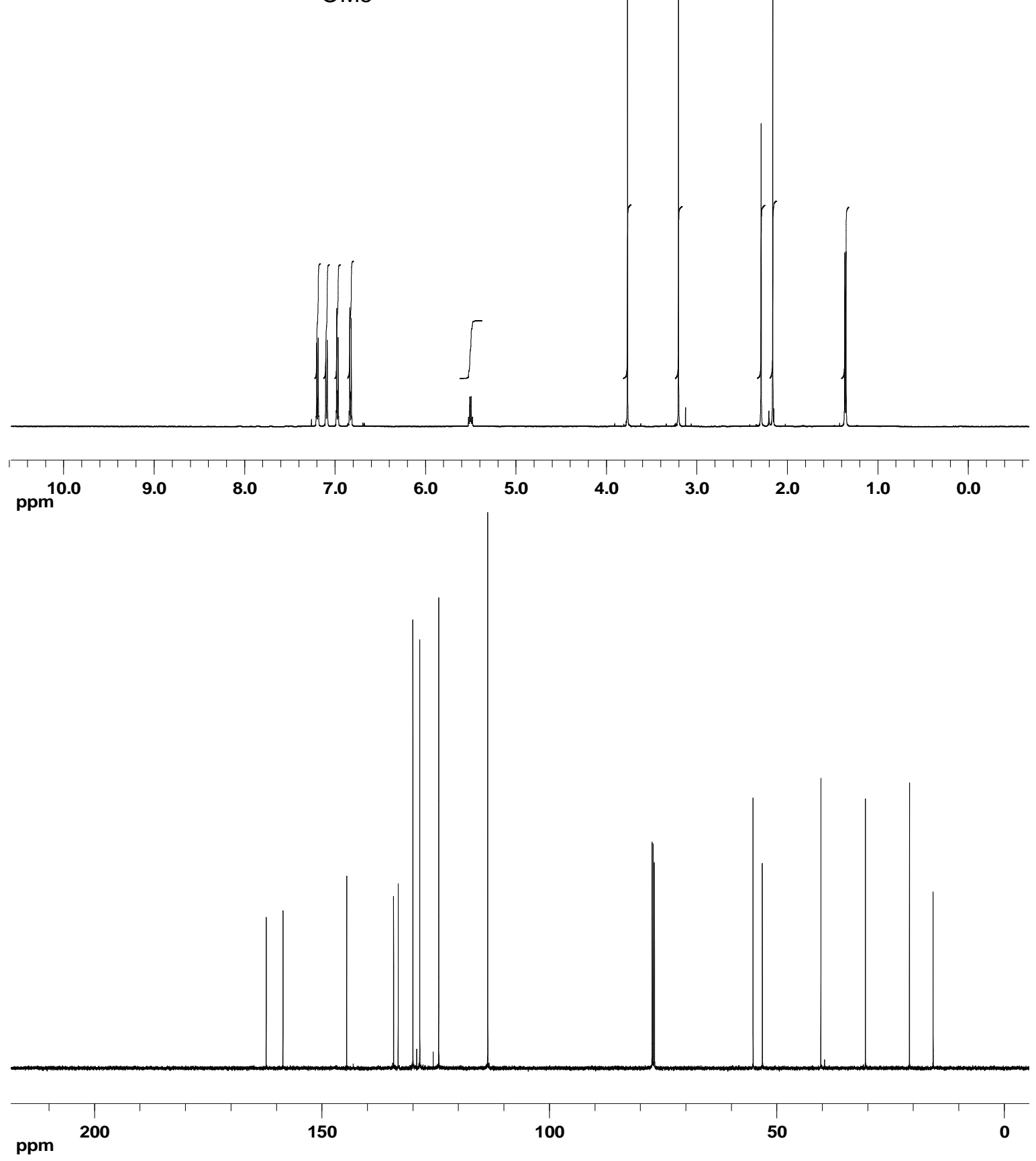
1-((S)-1-(4-Methoxyphenyl)ethyl)-1,3-dimethyl-3-(naphthalen-1-yl)urea 50<smiles>COc1ccc([C@H](C)N(C)C(=O)N(C)c2cccc3ccccc23)cc1</smiles>

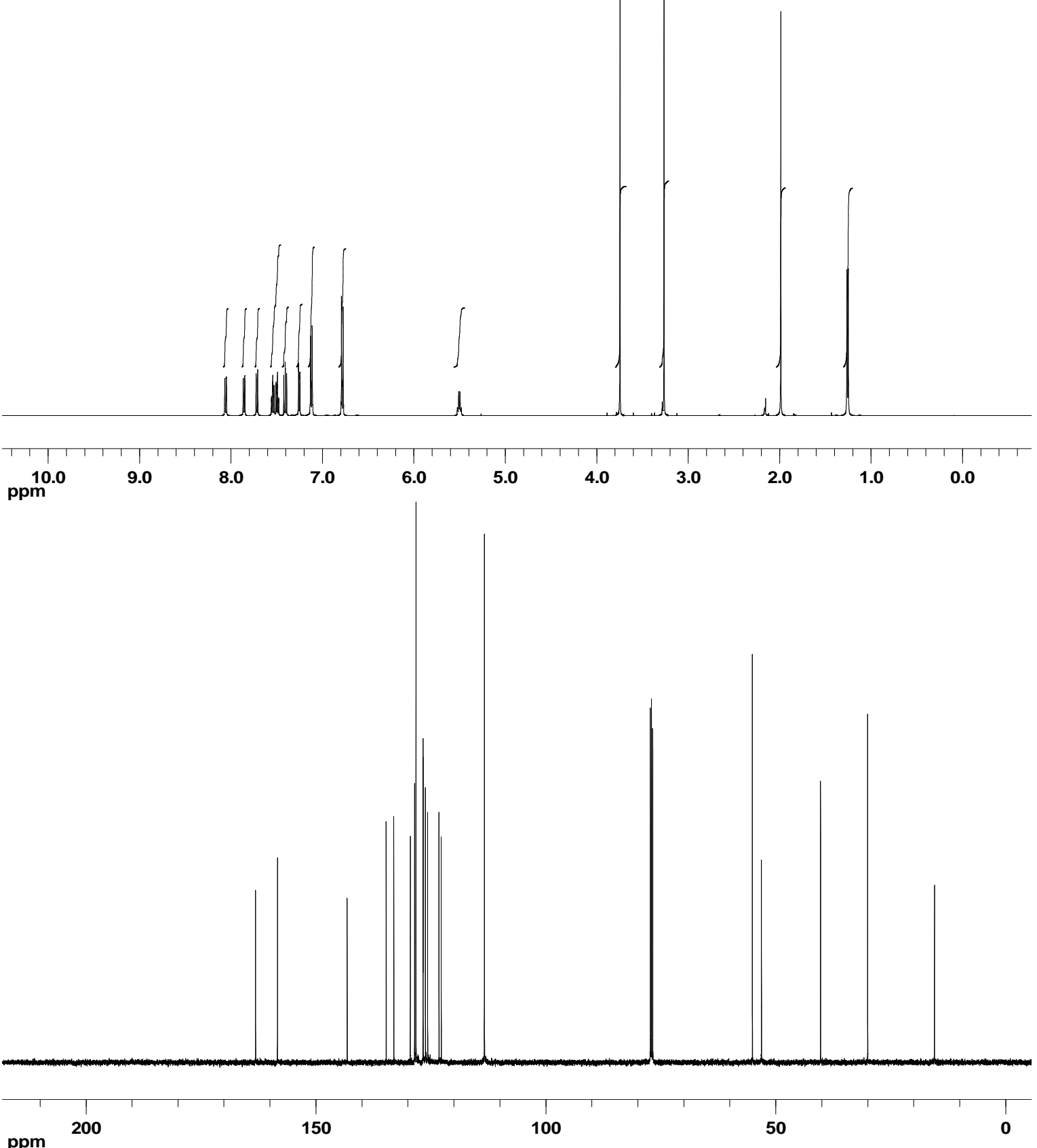


1-((R)-1-(4-Chlorophenyl)ethyl)-1,3-dimethyl-3-(naphthalen-1-yl)urea 5p<smiles>CC(c1ccc(Cl)cc1)N(C)C(=O)N(C)c1cccc2ccccc12</smiles>
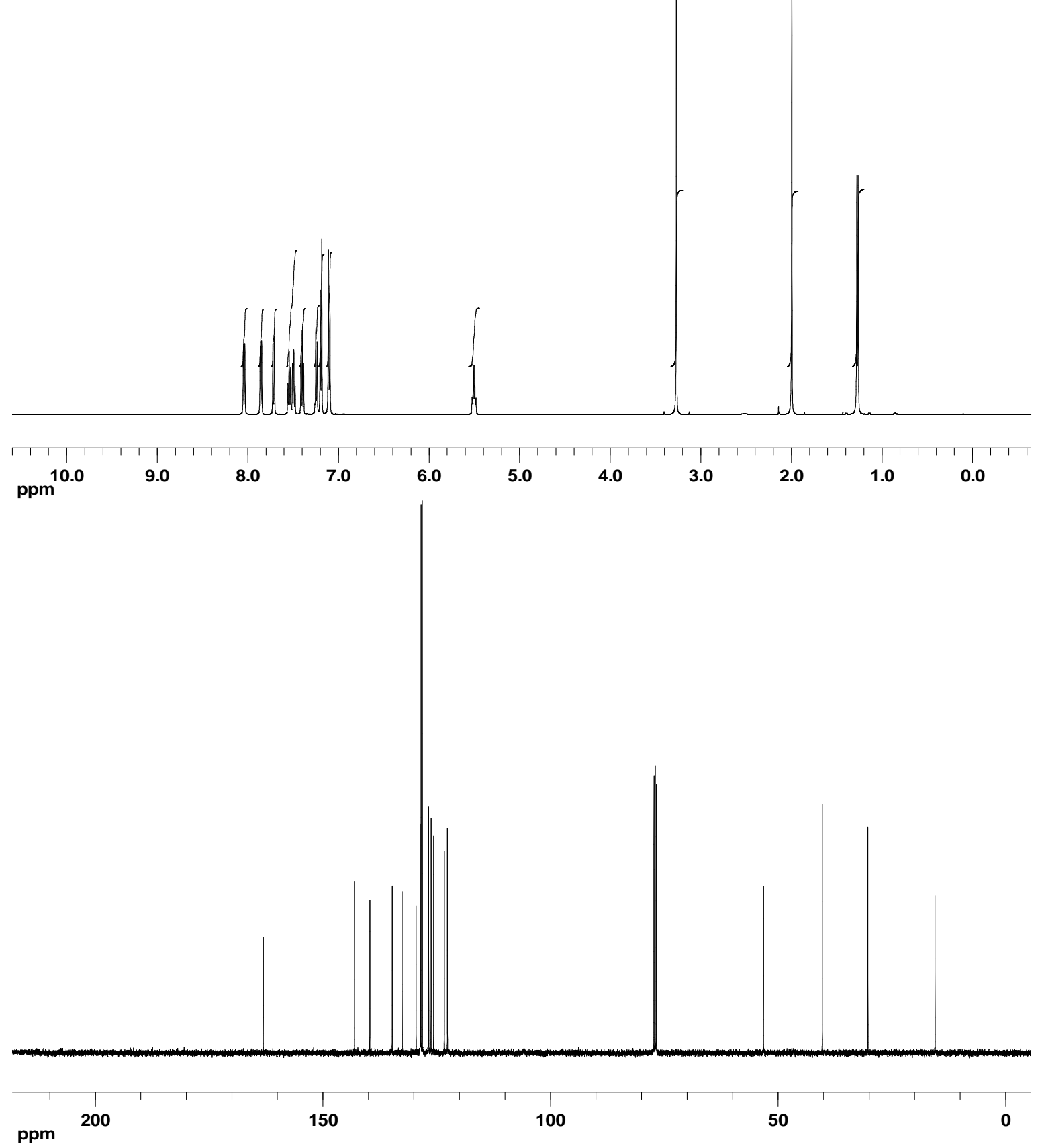

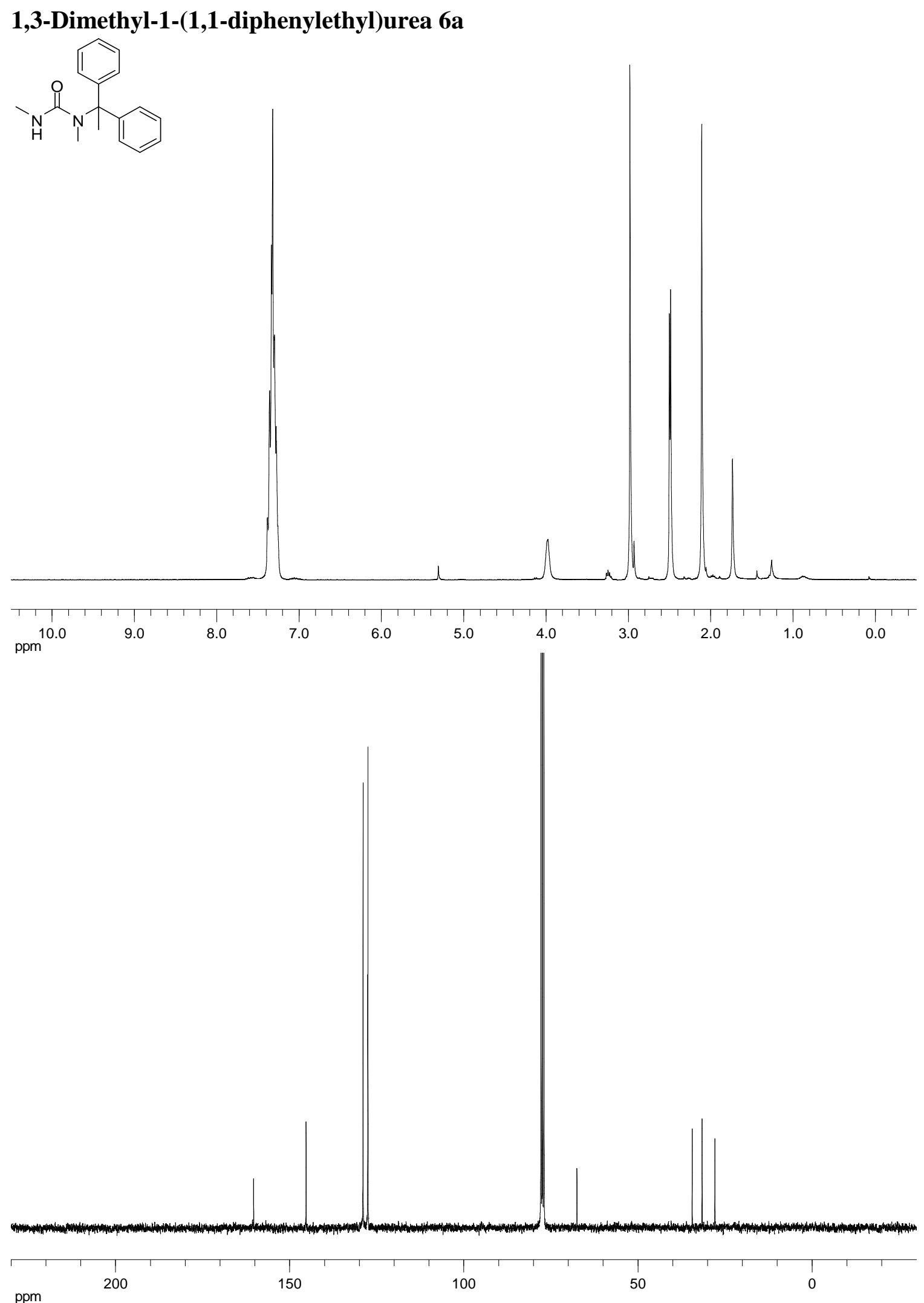

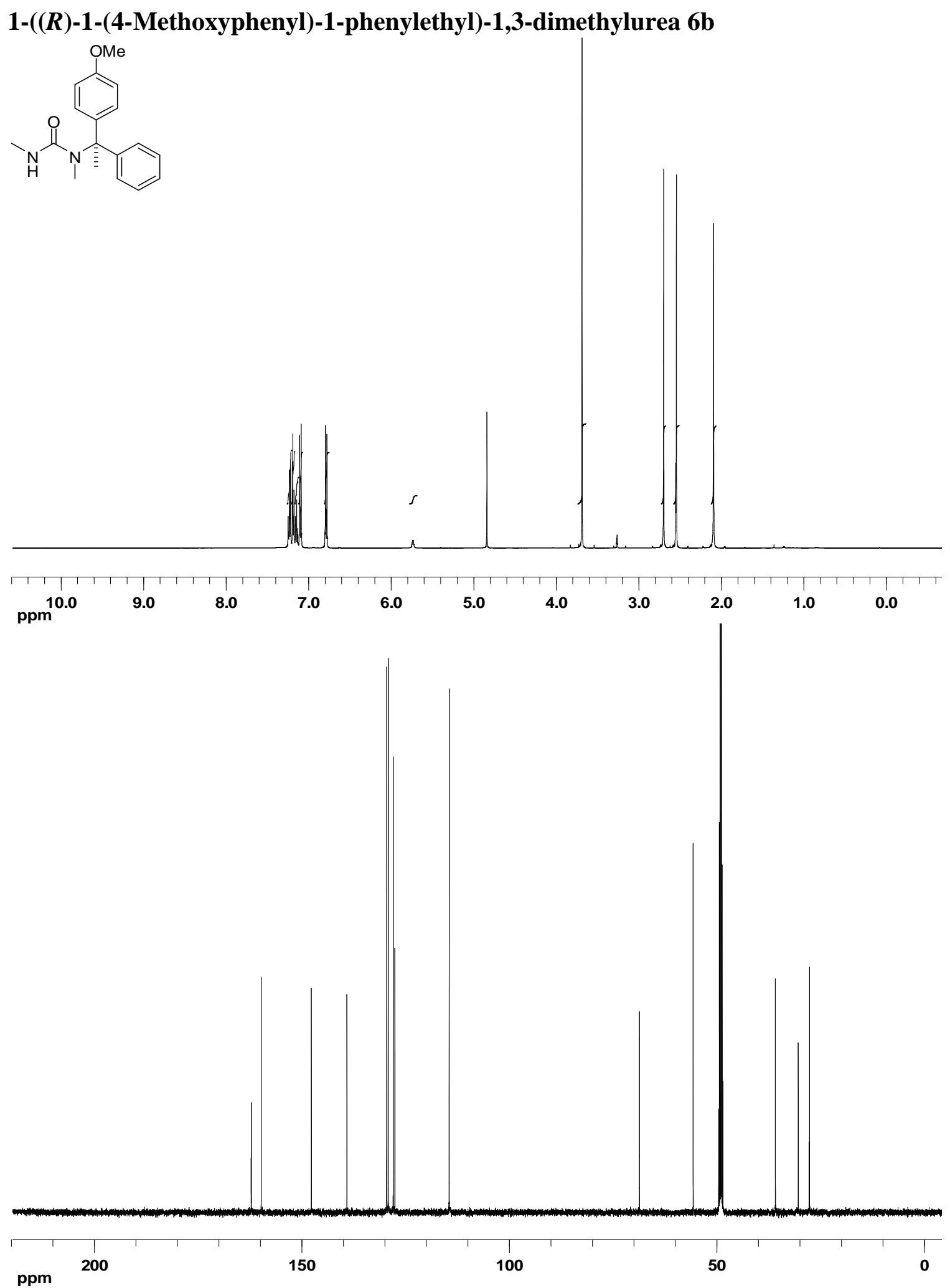
(R)-1-(1-(4-Methoxyphenyl)-1-phenylethyl)-1,3-dimethyl-3-nitrosourea 6b'<smiles>COc1ccc([C@H](c2ccccc2)N(C)C(=O)N(C)[N+](=O)[O-])cc1</smiles>
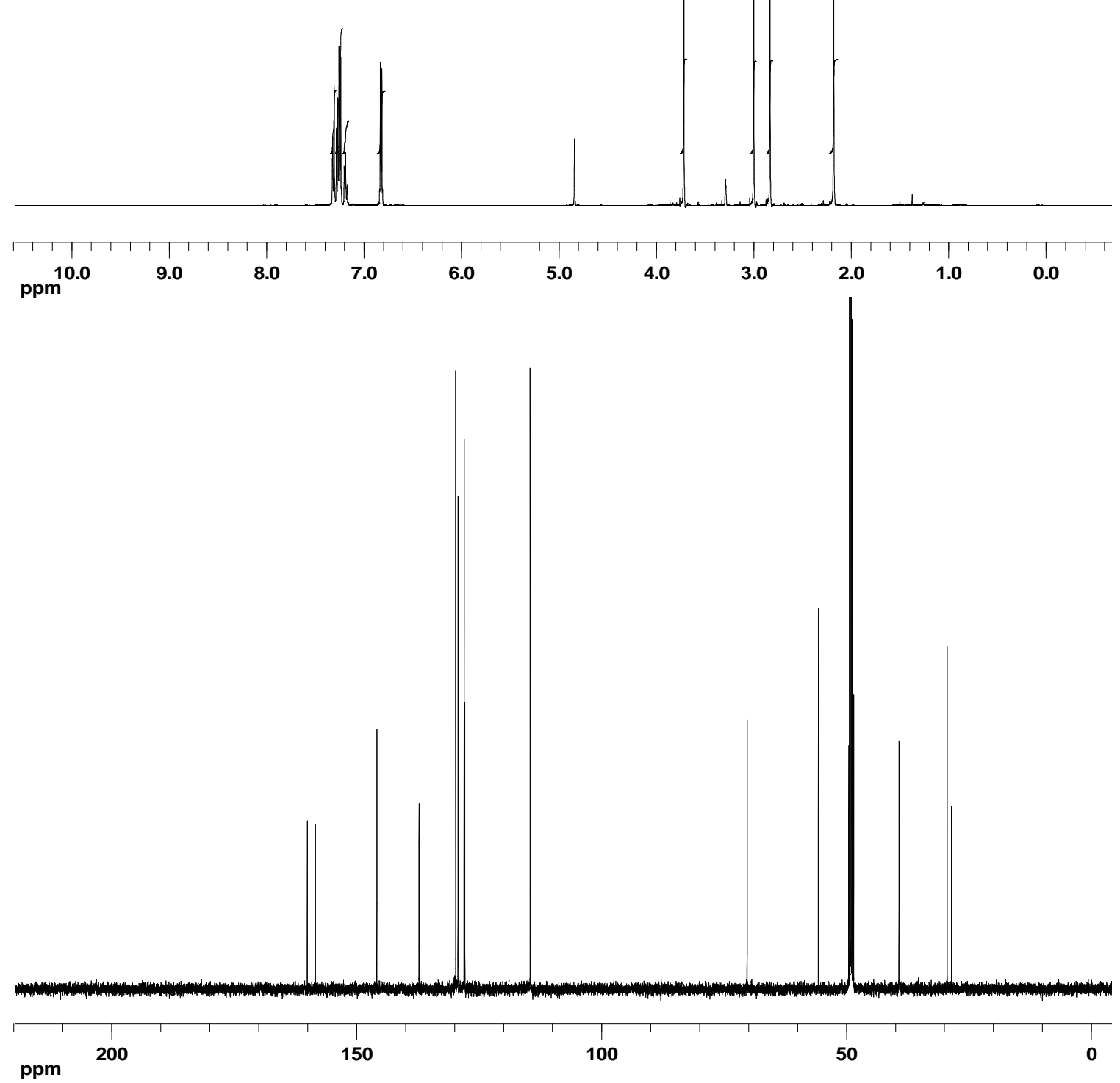

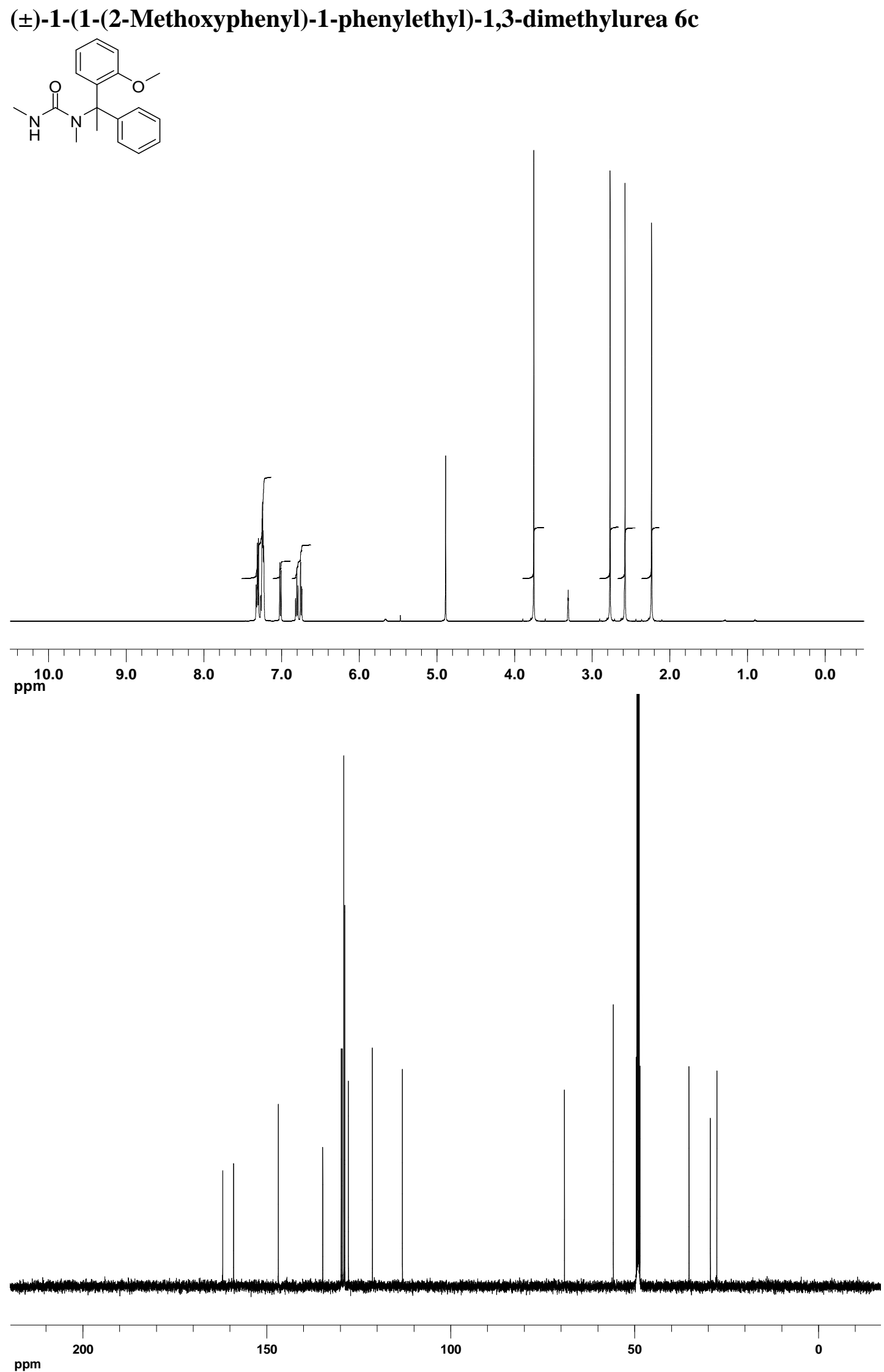


\section{1-((S)-1-(3,4-Dimethoxyphenyl)-1-phenylethyl)-1,3-dimethylurea 6d}
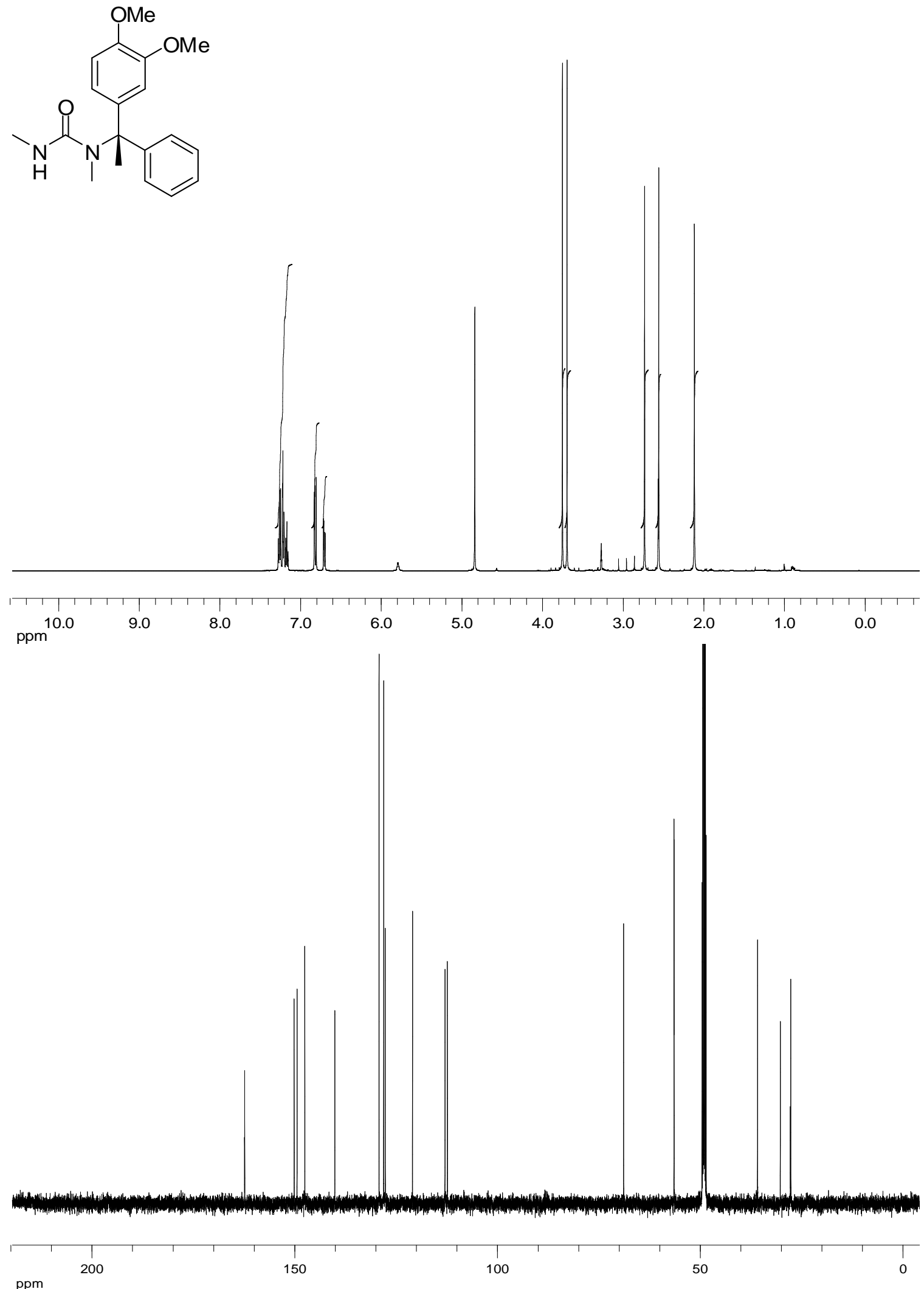
(士)-1-(1-(2-Fluorophenyl)-1-phenylethyl)-1,3-dimethylurea 6e
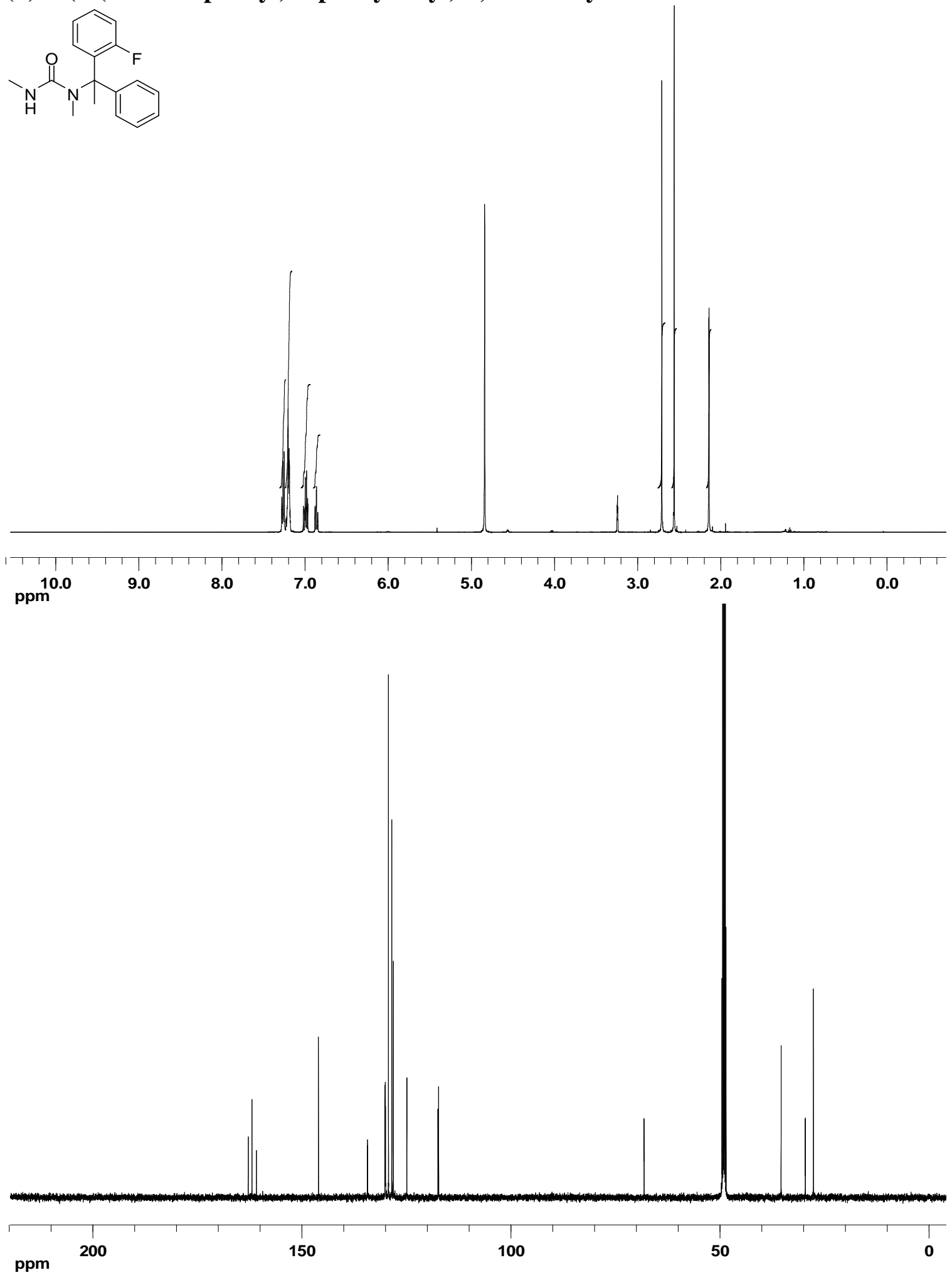

45 
( \pm )-1-(1-(3-Fluorophenyl)-1-phenylethyl)-1,3-dimethylurea 6f
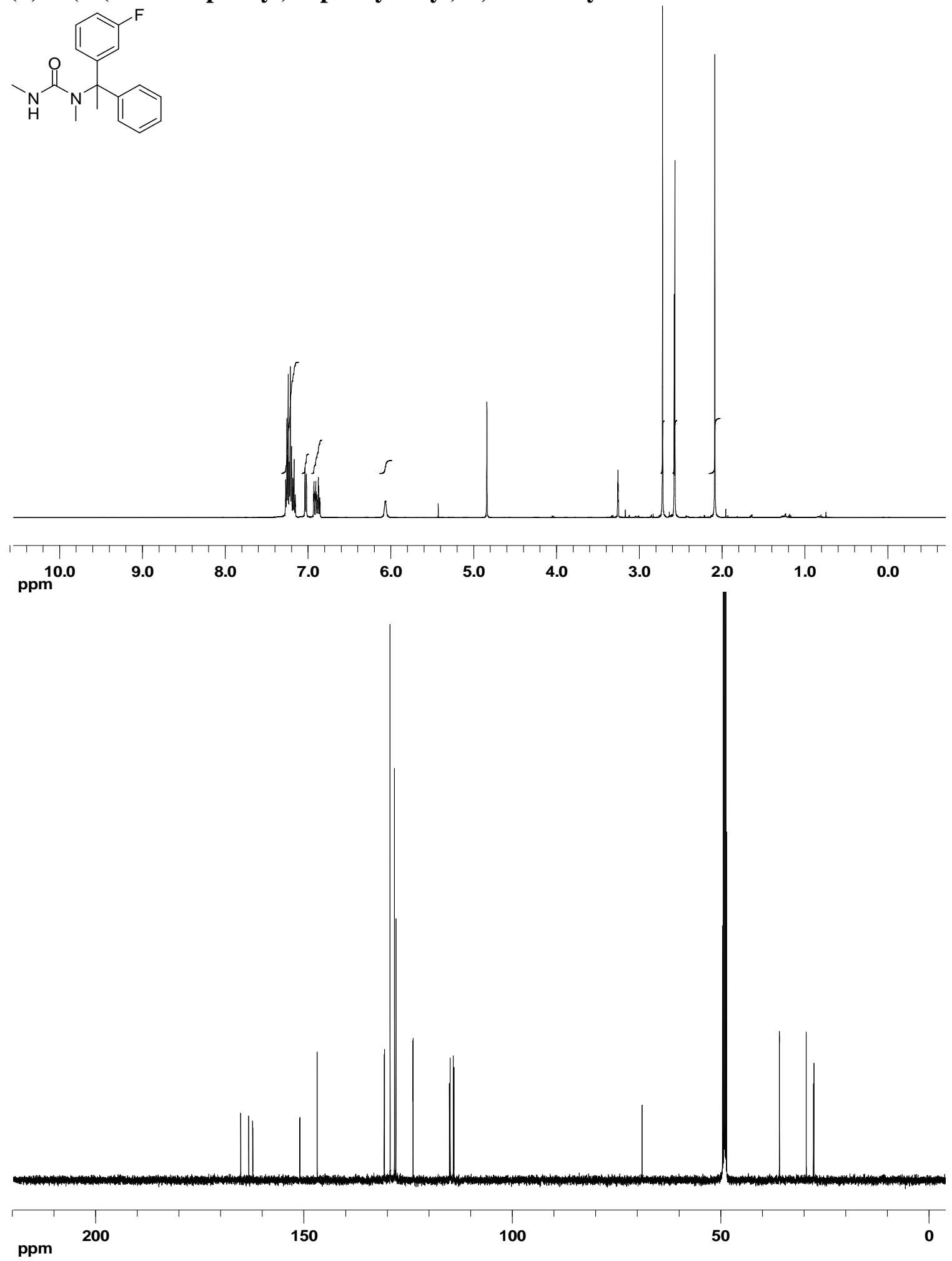
1-((S)-1-(4-Fluorophenyl)-1-phenylethyl)-1,3-dimethylurea 6g
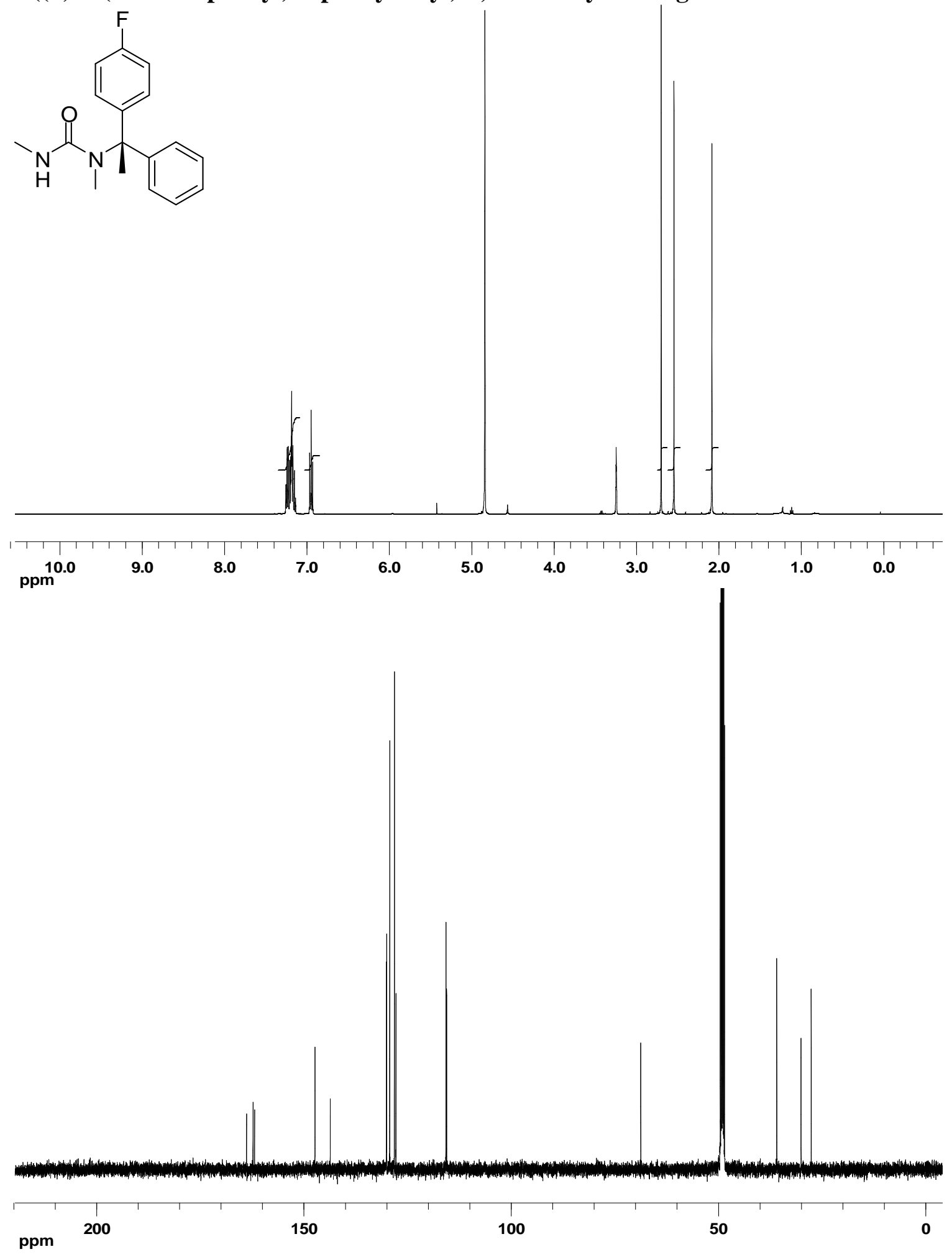

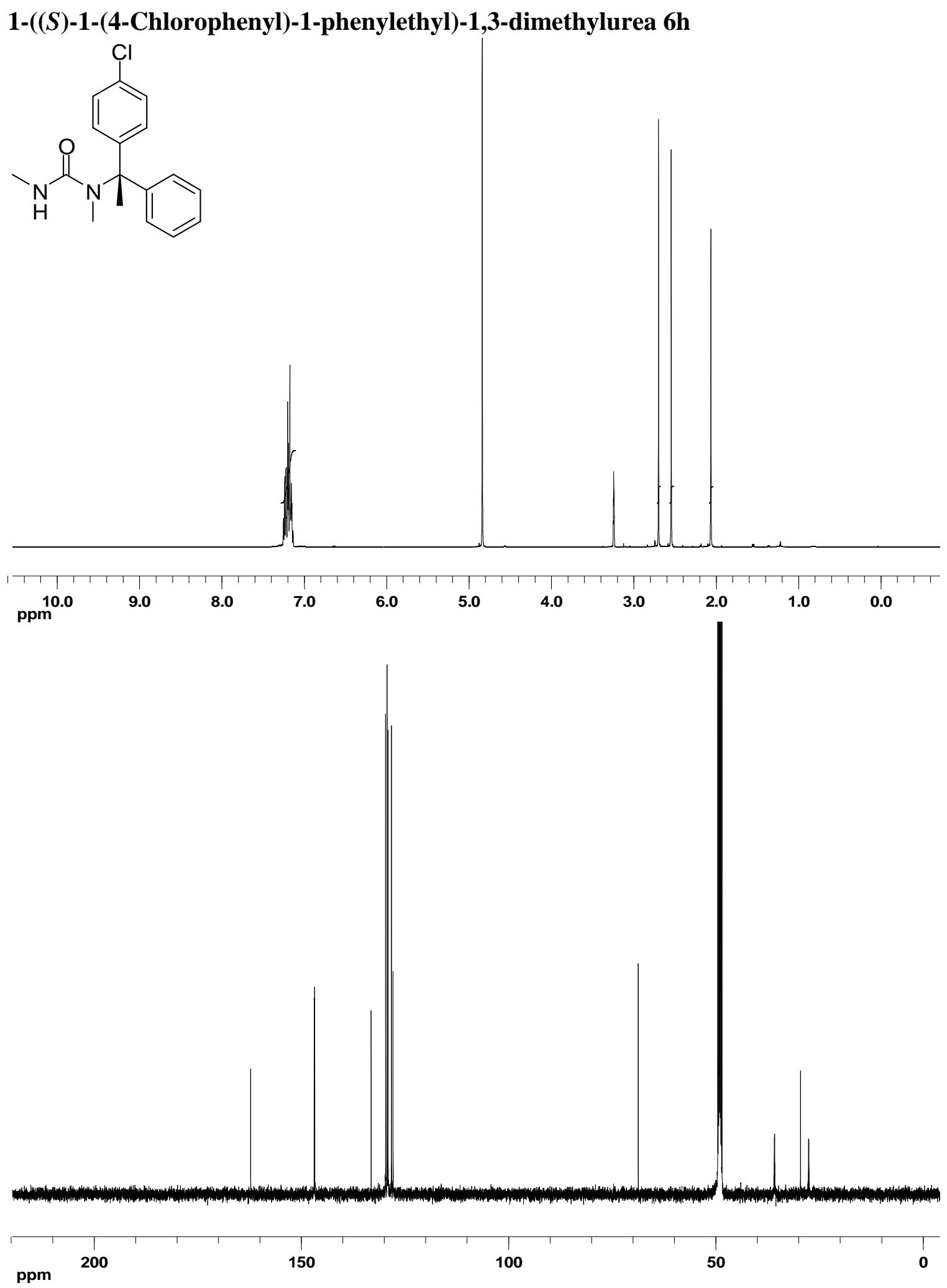


\section{1-((S)-1-(3-Chloro-4-fluorophenyl)-1-phenylethyl)-1,3-dimethylurea 6i}
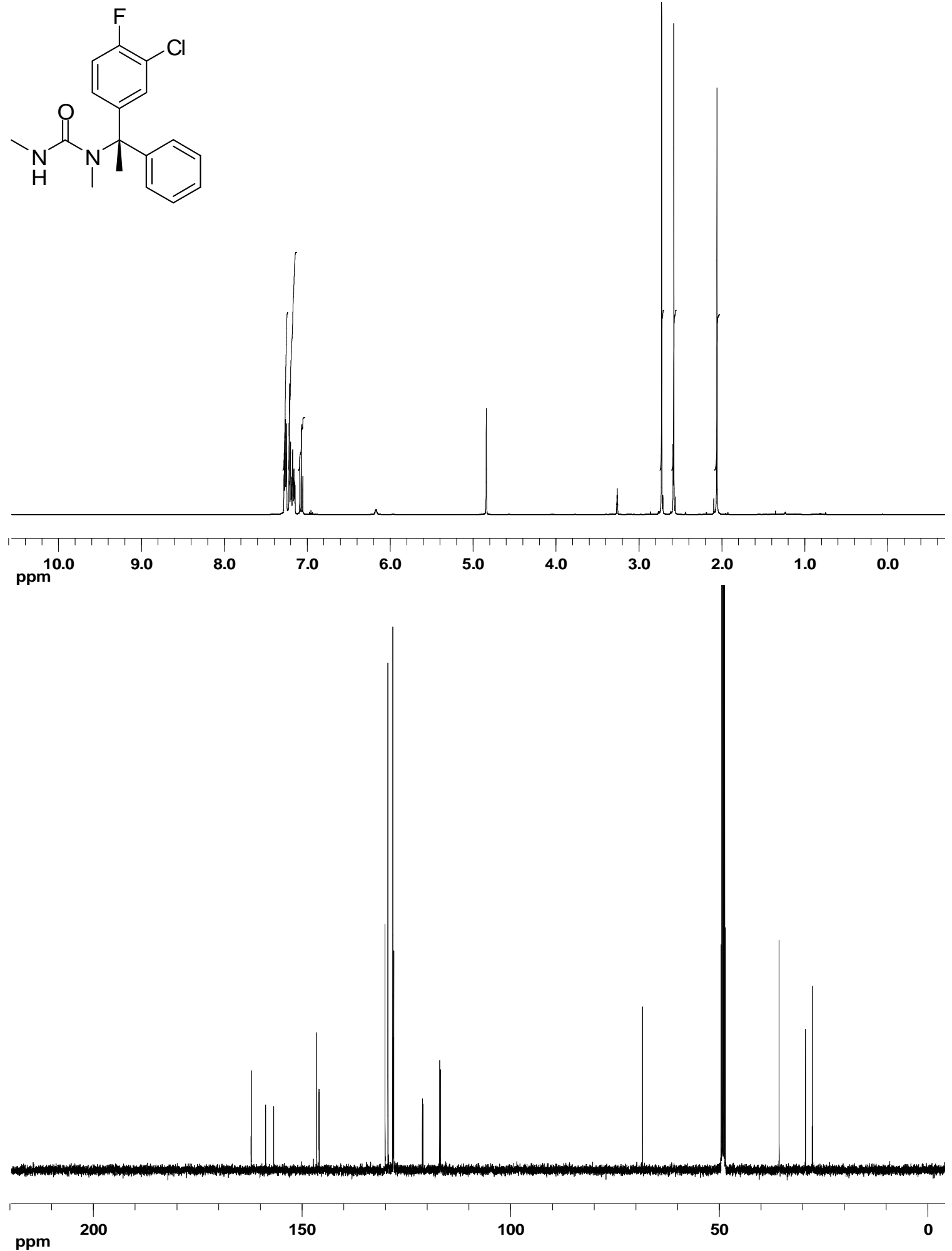


\section{1,1,3-Trimethyl-3-((S)-1-(naphthalen-1-yl)-1-phenylethyl)urea 6j}
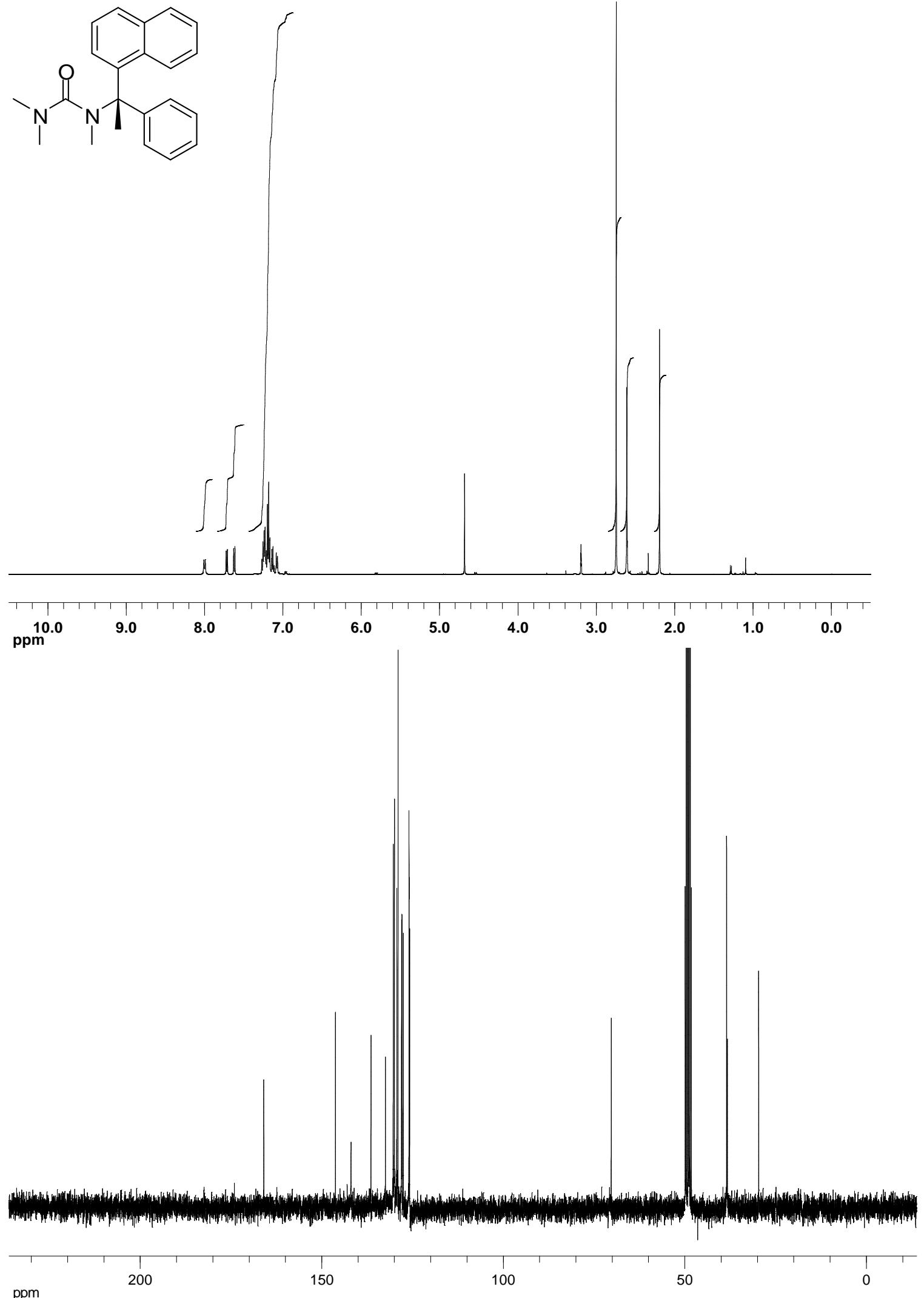


\section{1,1,3-Trimethyl-3-((S)-1-(naphthalen-2-yl)-1-phenylethyl)urea 6k}<smiles>CN(C)C(=O)N(C)[C@@](C)(c1ccccc1)c1ccc2ccccc2c1</smiles>
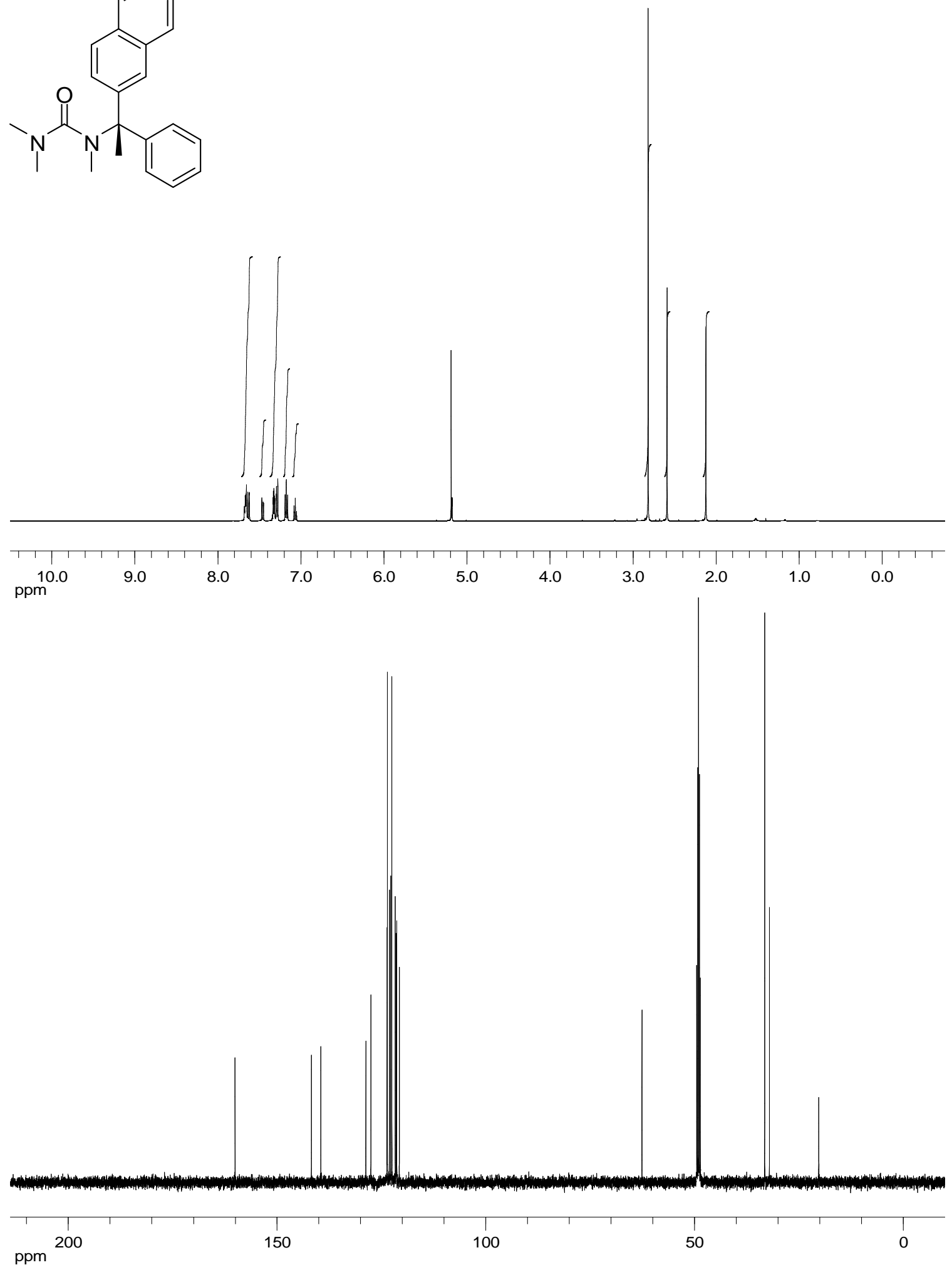


\section{1-((S)-1-(4-Methoxyphenyl)-1-o-tolylethyl)-1,3-dimethylurea 6m}<smiles>CNC(=O)N(C)[C@@](C)(c1ccc(OC)cc1)c1ccccc1C</smiles>
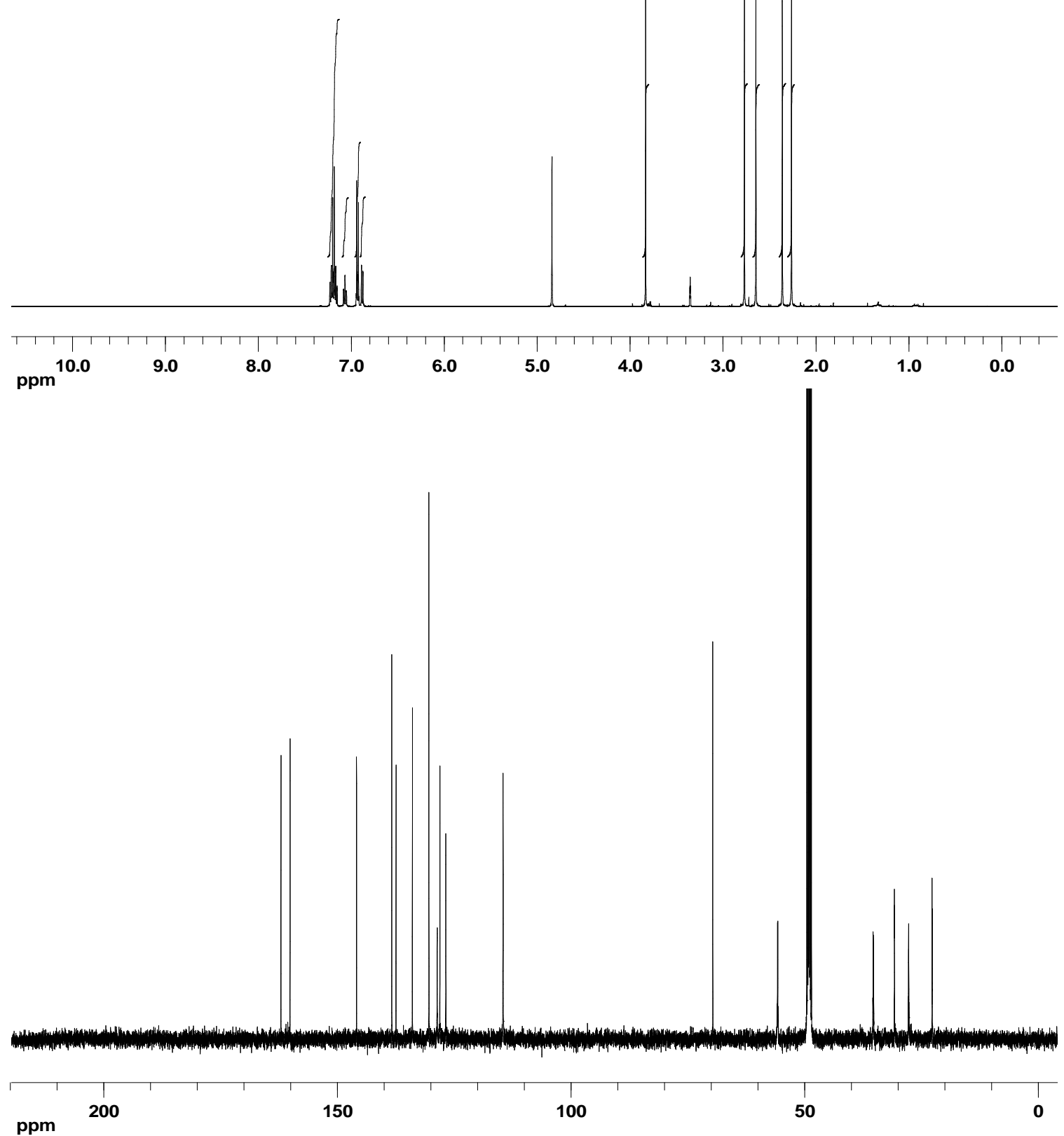

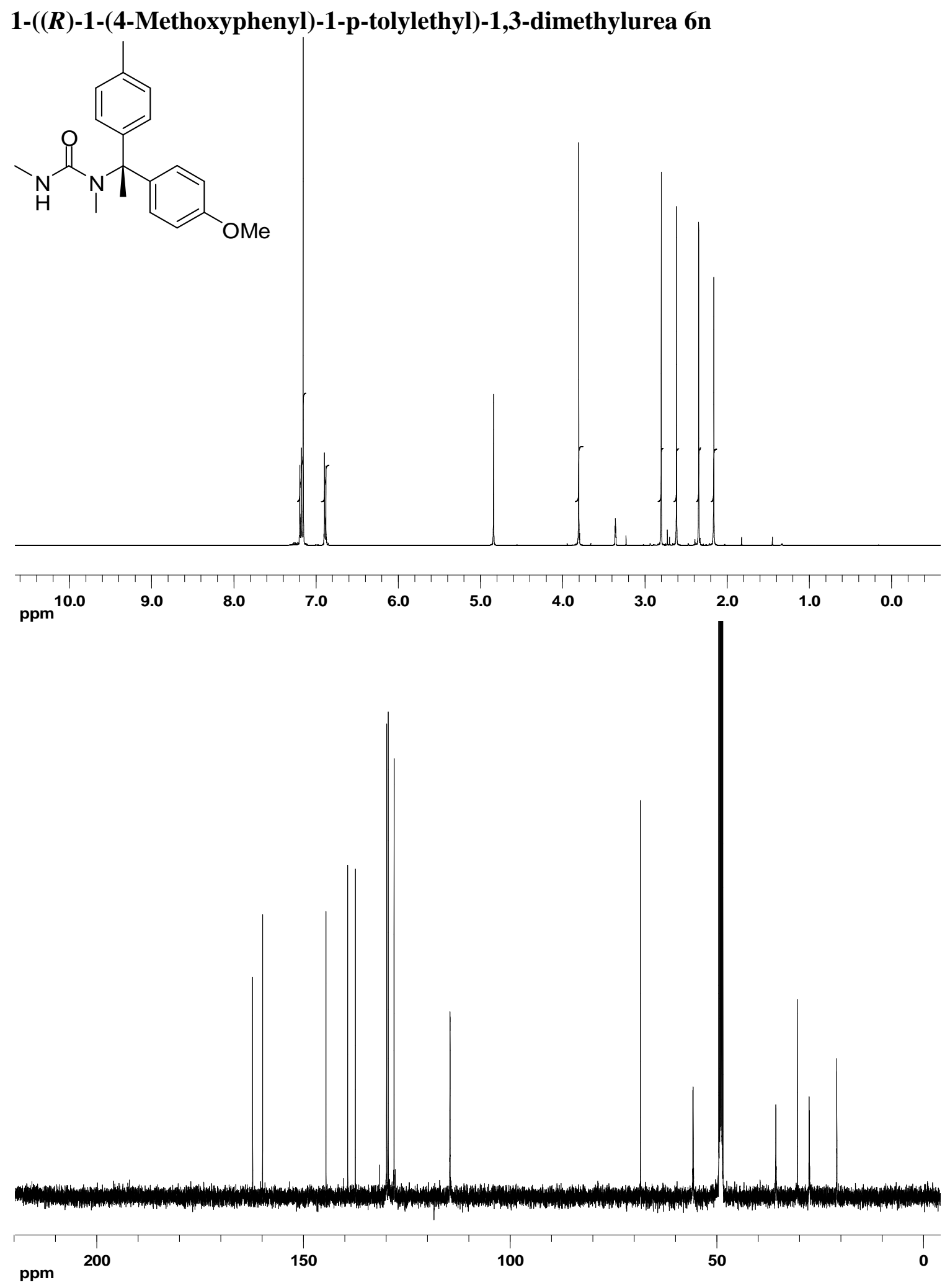
1-((S)-1-(4-Methoxyphenyl)-1-(naphthalen-1-yl)ethyl)-1,3,3-trimethylurea 60
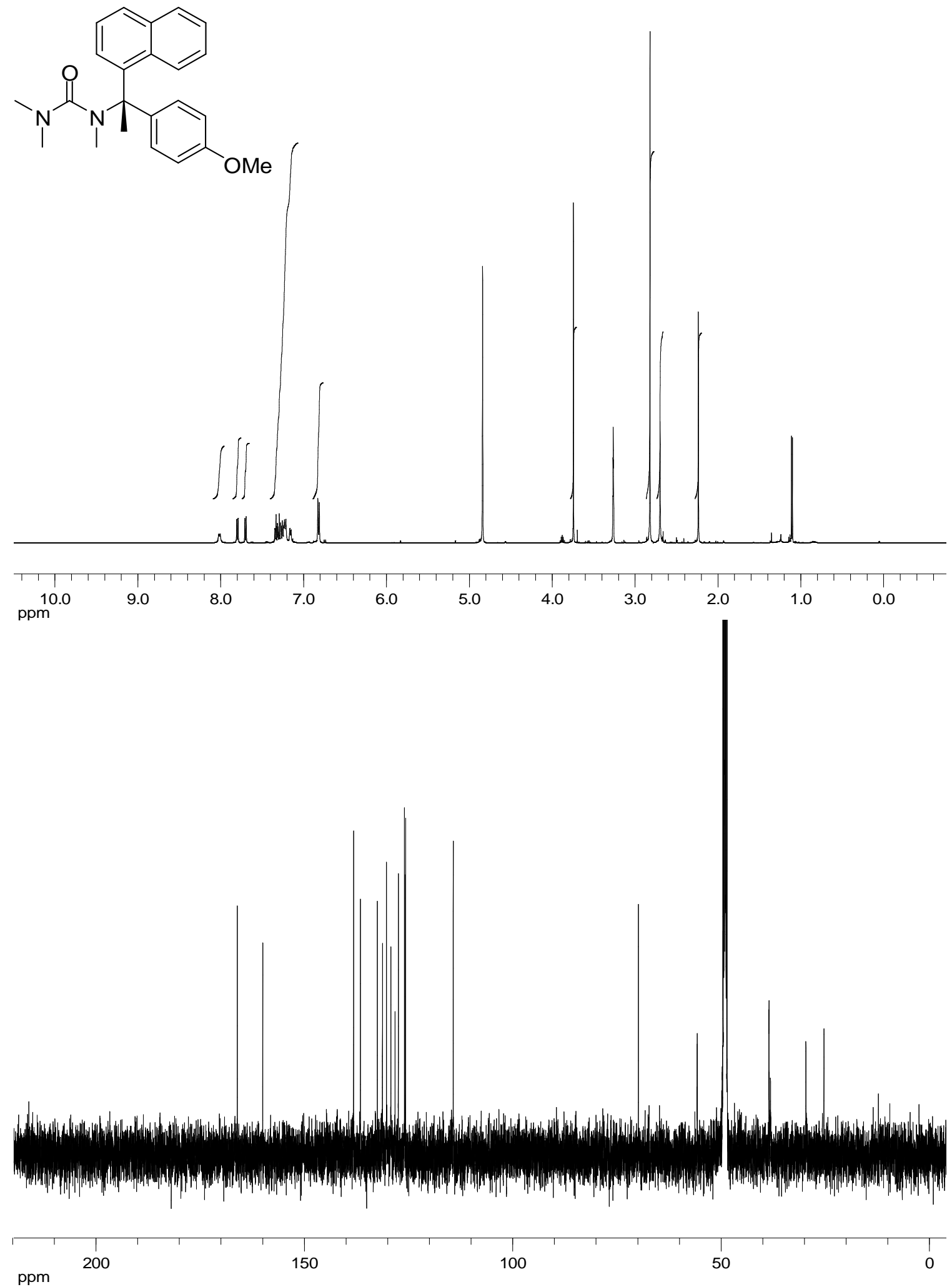

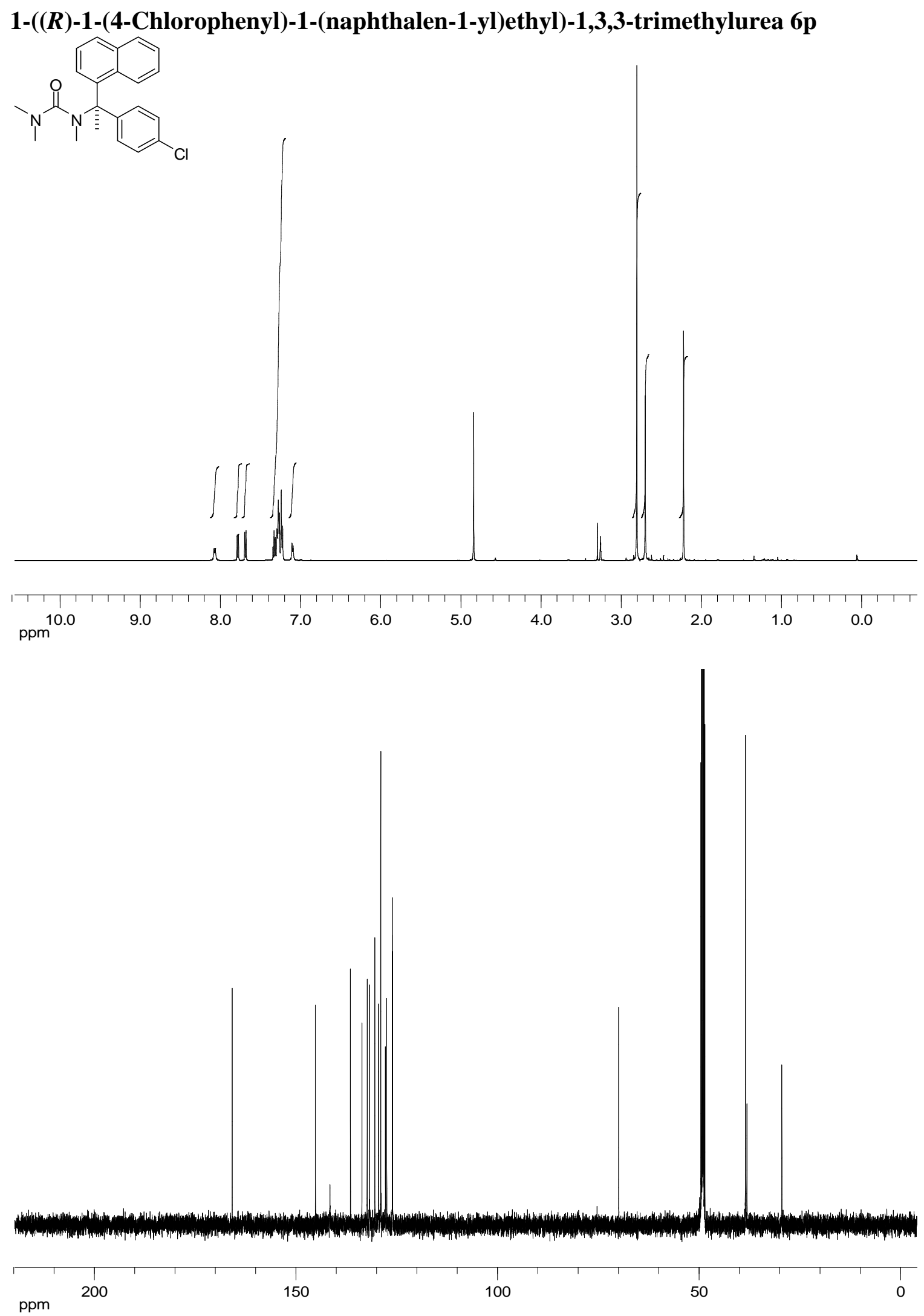
$N$-Methyl-1,1-diphenylethanamine 7a
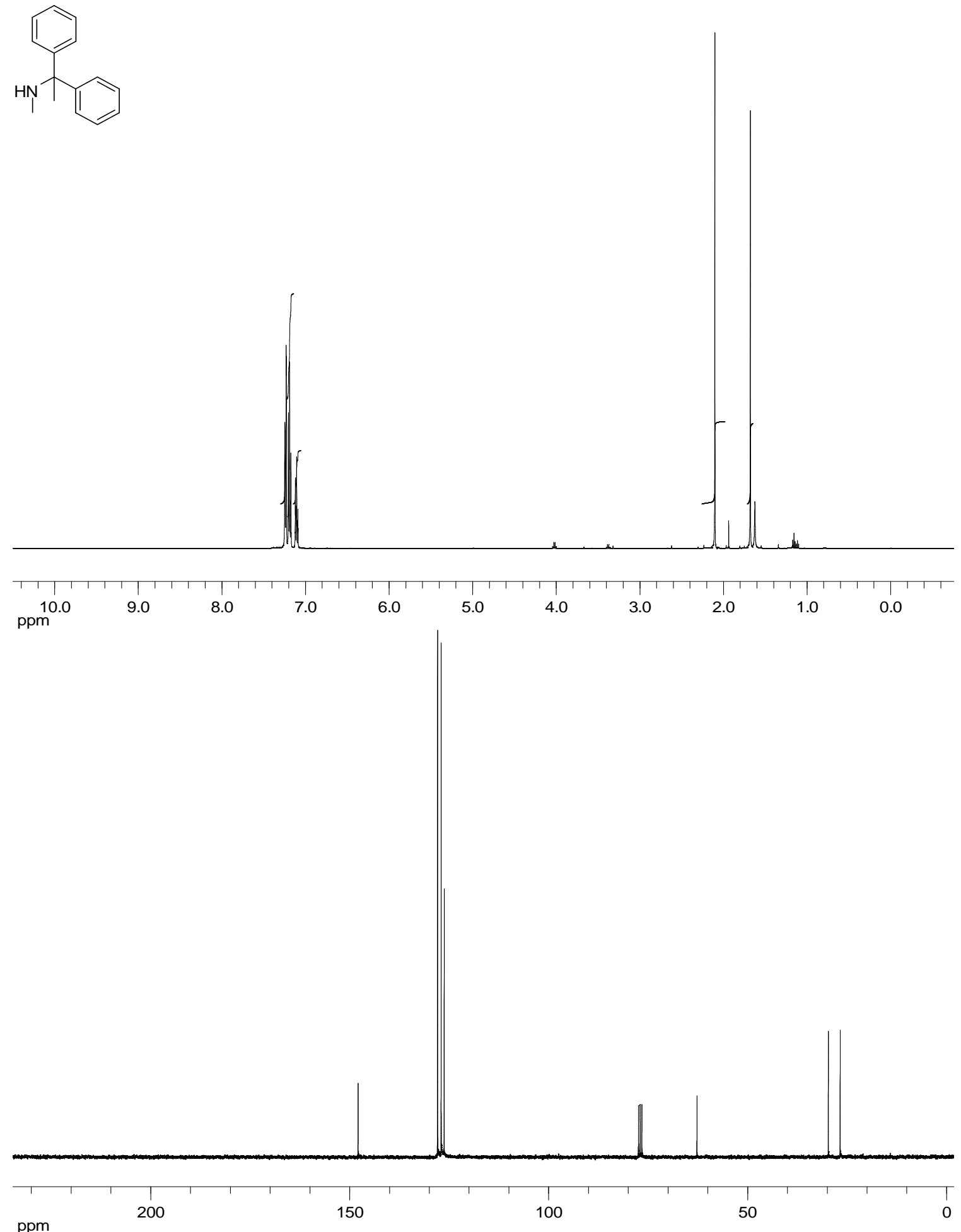

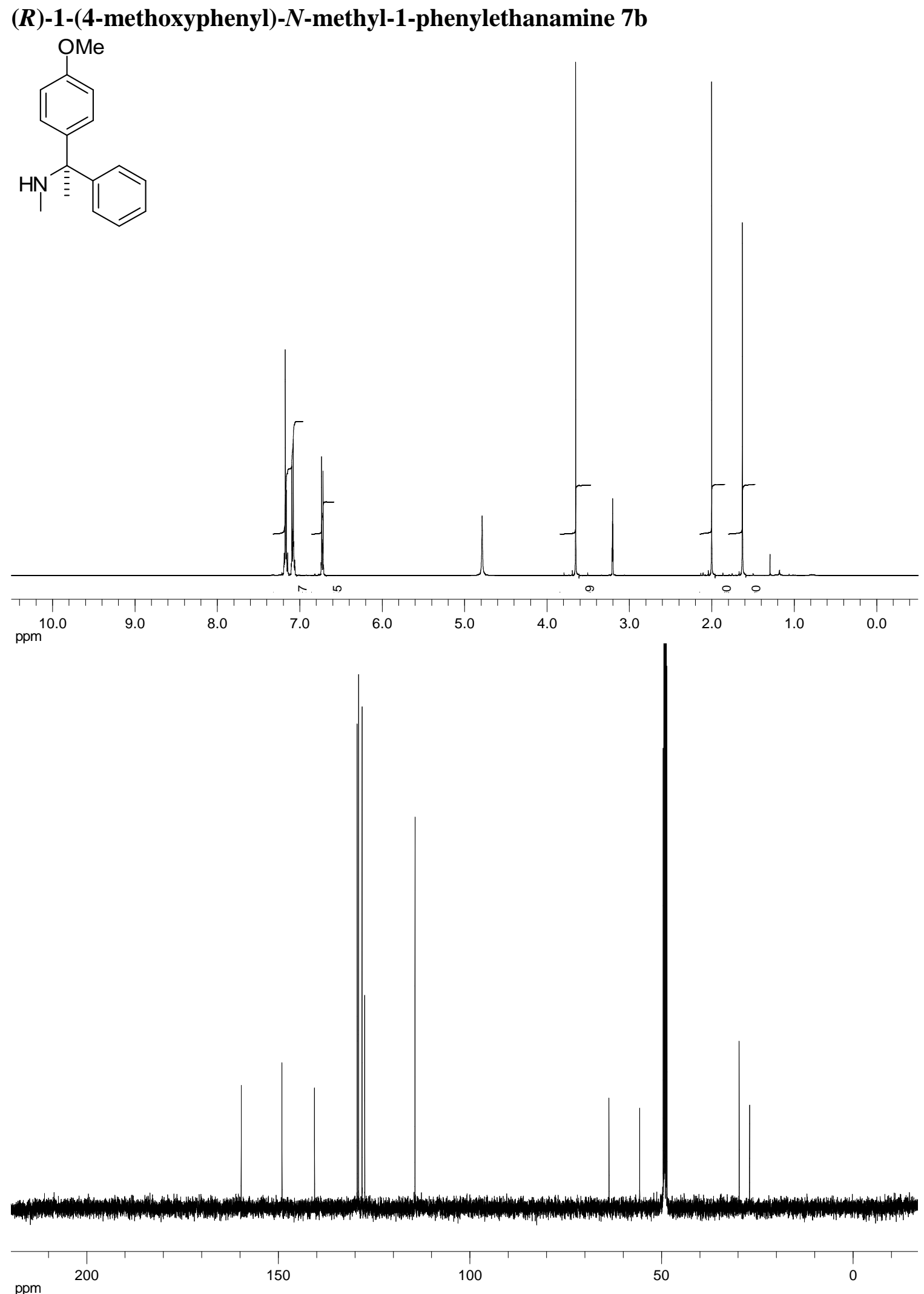
(S)-1-(3-Chloro-4-fluorophenyl)- $N$-methyl-1-phenylethanamine 7i
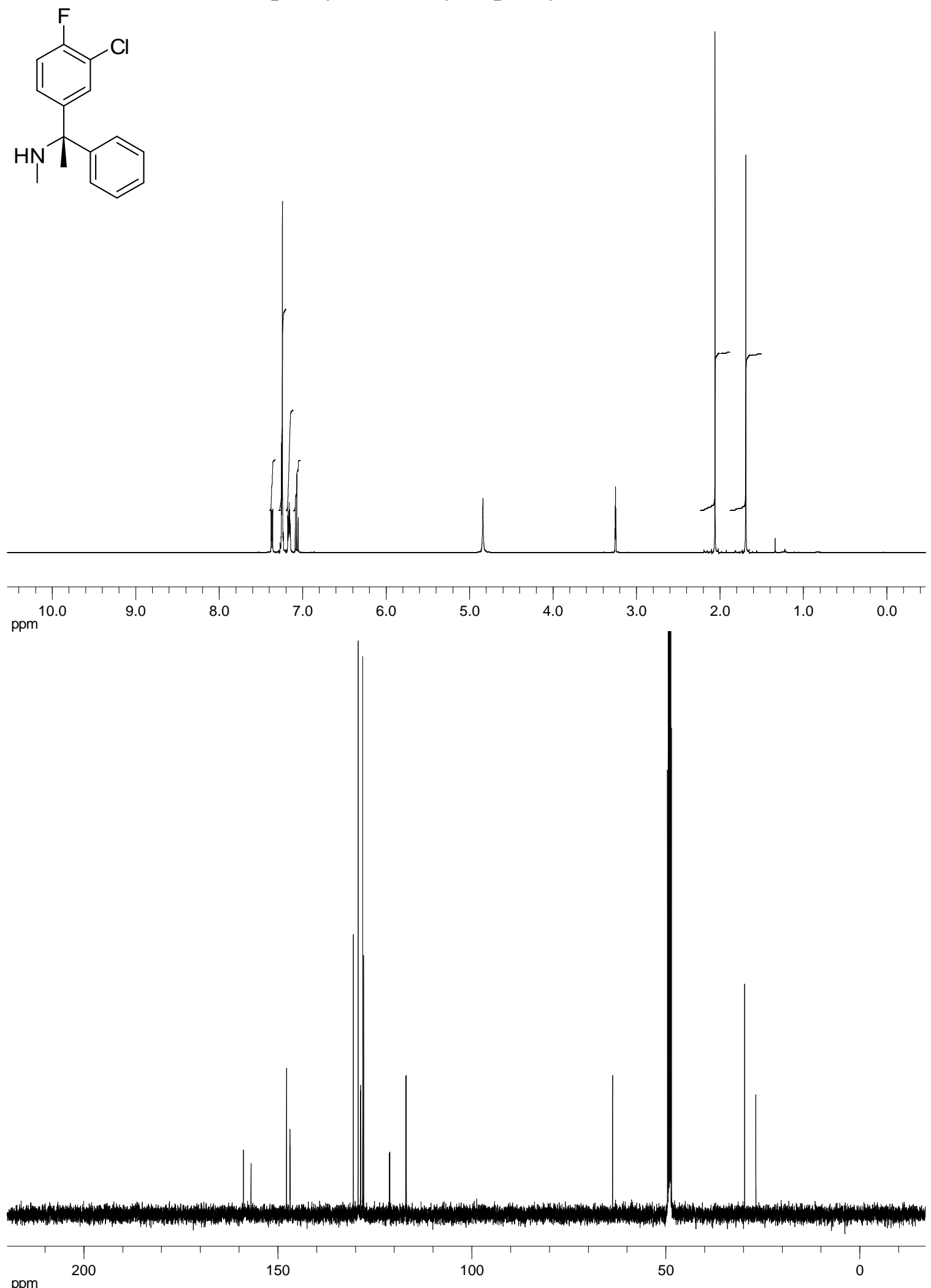
(S)-N-Methyl-1-(naphthalen-1-yl)-1-phenylethanamine 7j<smiles>CNC(C)(c1ccccc1)c1cccc2ccccc12</smiles>
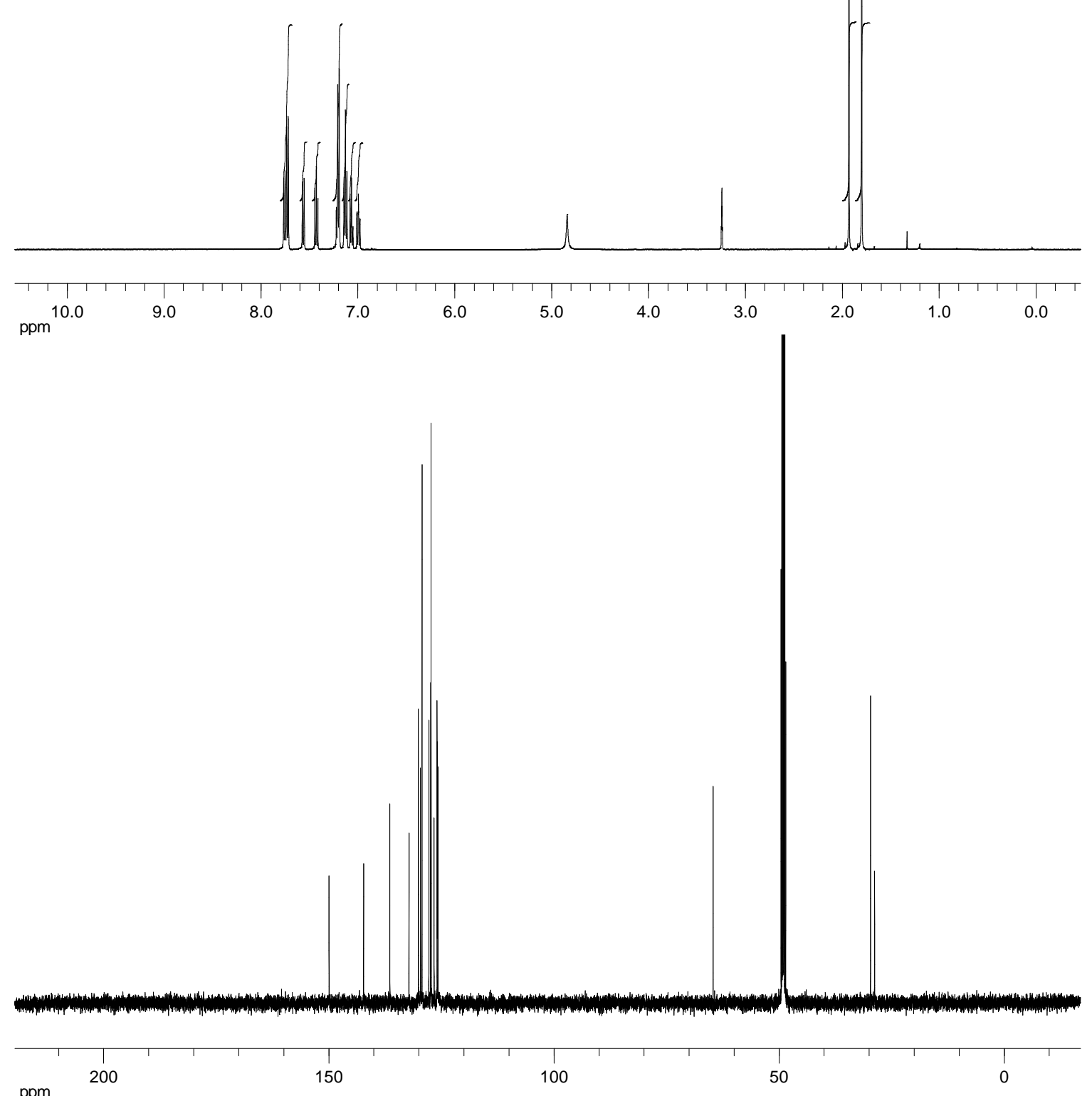
(1'R,5R)-5-(4-Methoxyphenyl)-1,3,5-trimethyl-4'H-spiro[imidazolidine-4,1'naphthalene]-2,4'-dione 100<smiles>COc1ccc([C@]2(C)N(C)C(=O)N(C)[C@@]23C=CC(=O)c2ccccc23)cc1</smiles>
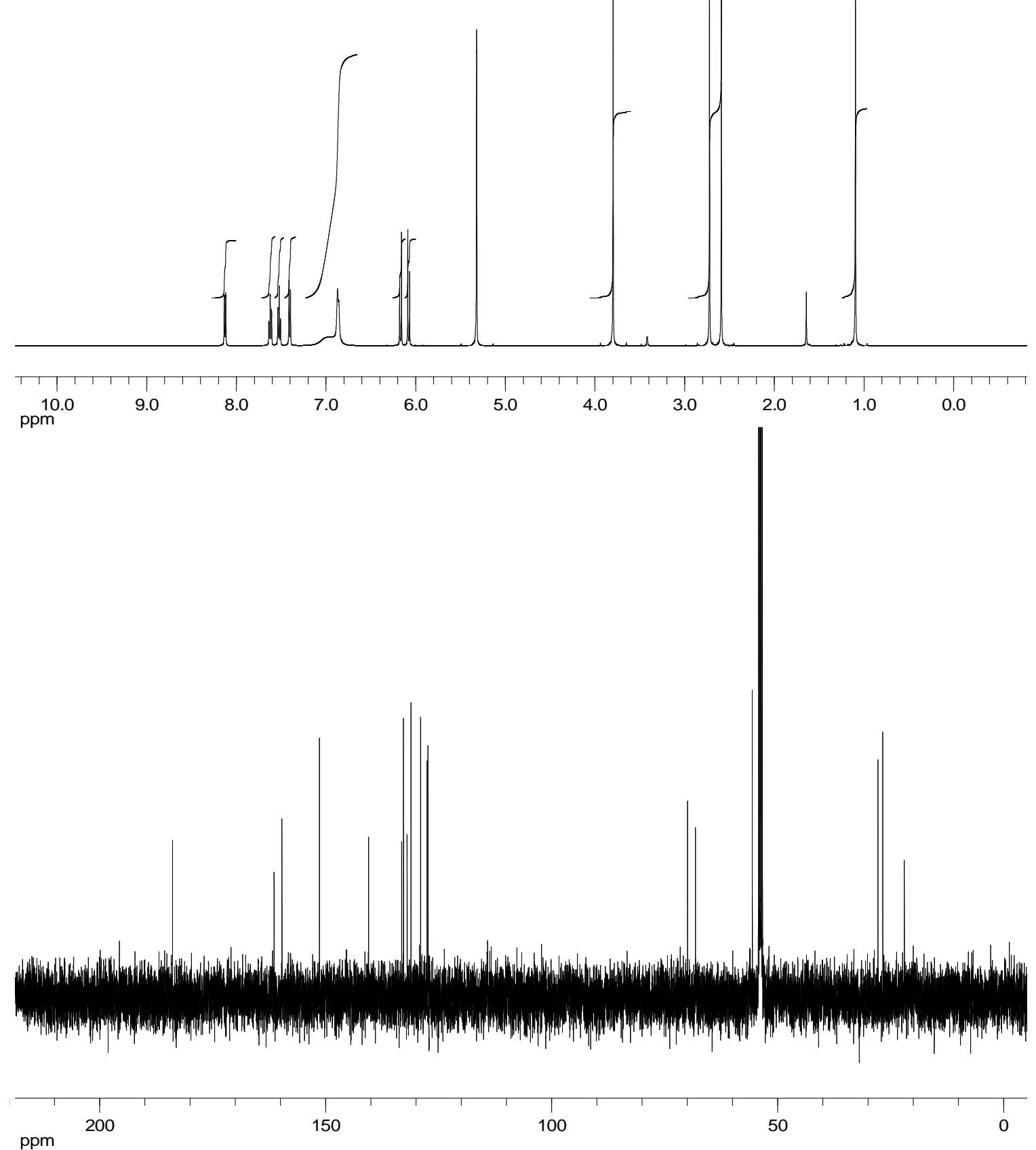
(1'R,5R)-5-(4-Chlorophenyl)-1,3,5-trimethyl-4'H-spiro[imidazolidine-4,1'naphthalene]-2,4'-dione 10p
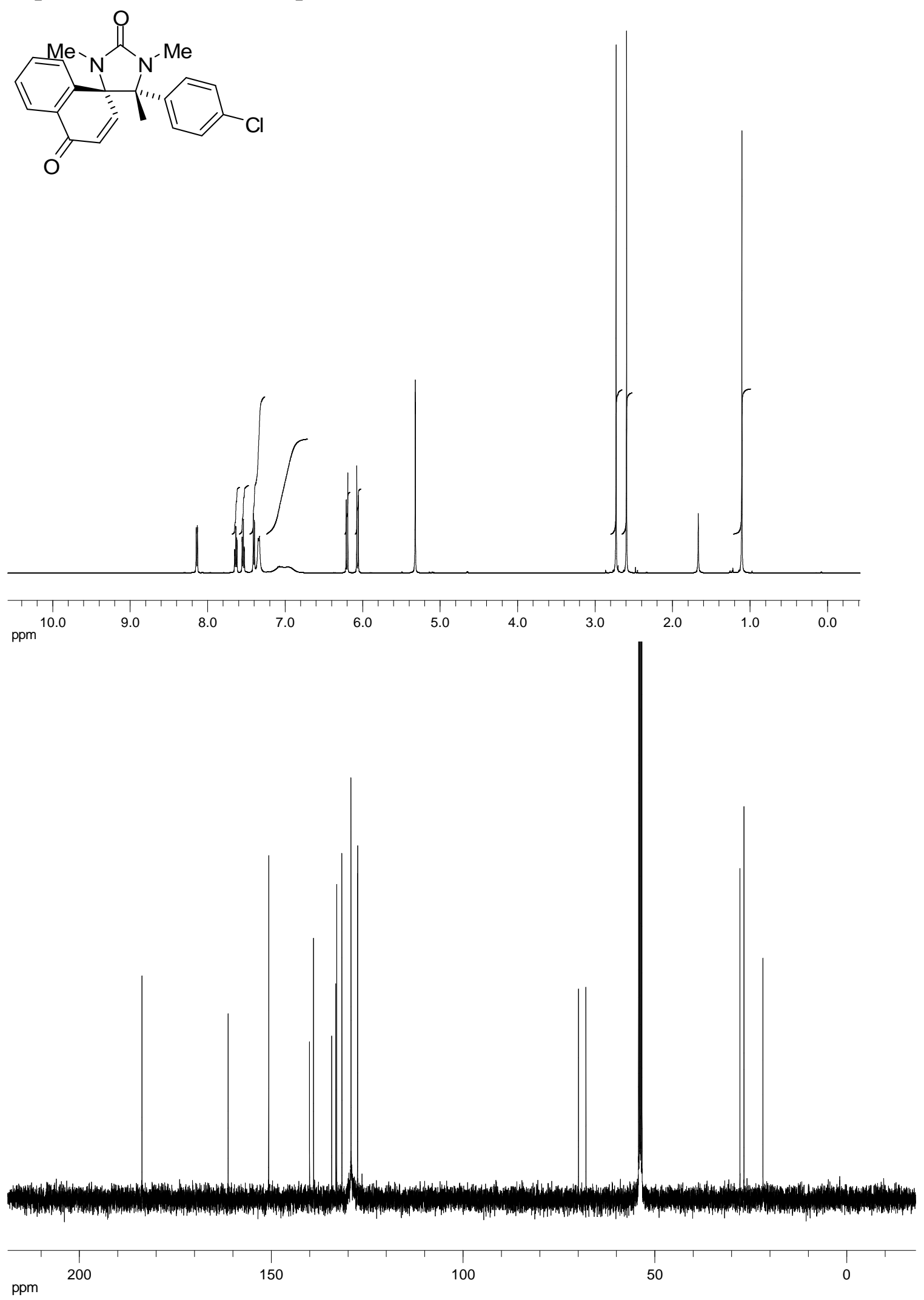
(士)-1,3-Dimethyl-3-nitroso-1-(phenyl(o-tolyl)methyl)urea $N$-nitroso-3e<smiles>Cc1ccccc1C(c1ccccc1)N(C)C(=O)N(C)[N+](=O)[O-]</smiles>
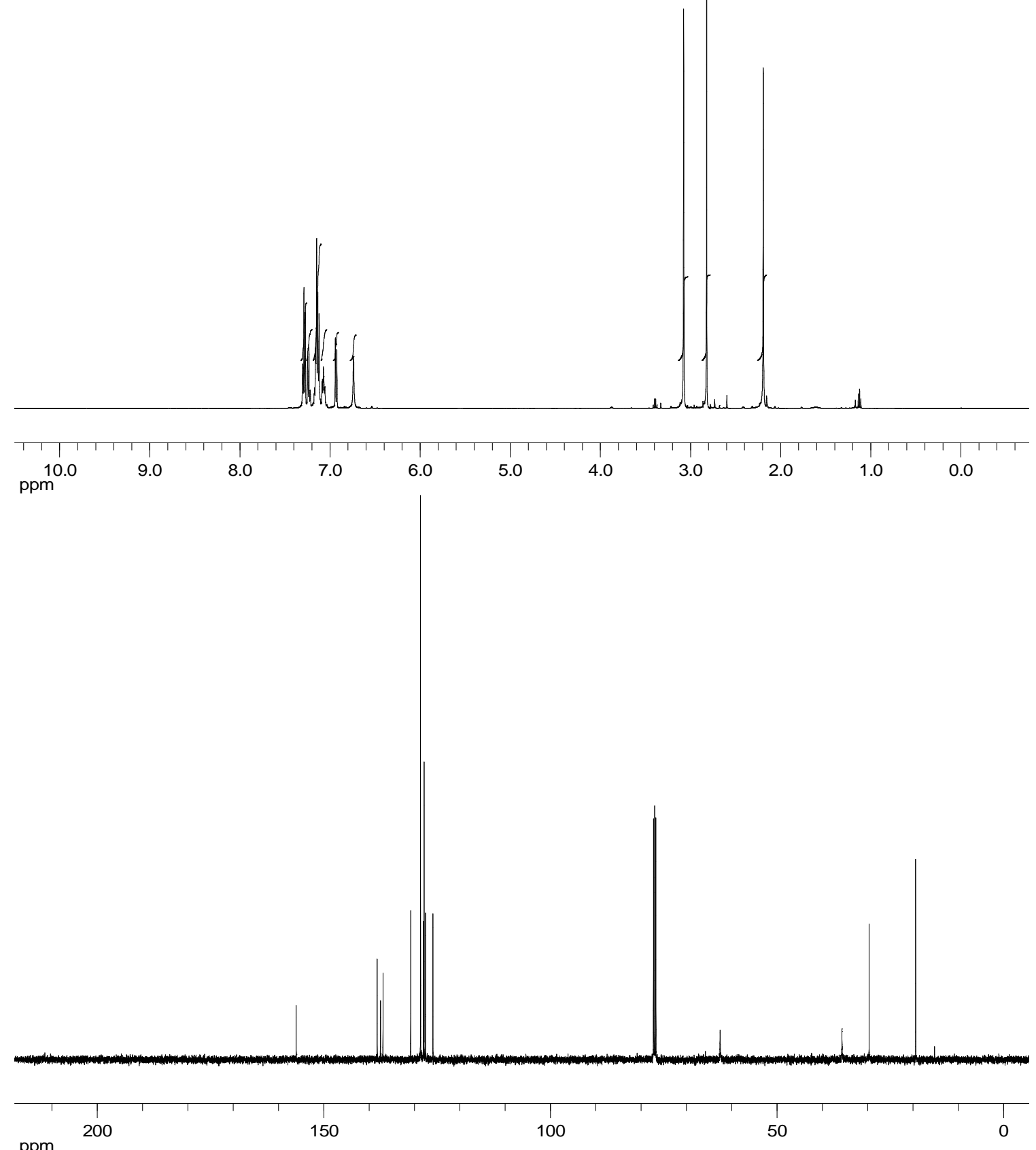
( \pm )-1-((2-Methoxyphenyl)benzyl)-1,3-dimethyl-3-nitrosourea $N$-nitroso-3f<smiles>COc1ccccc1C(c1ccccc1)N(C)C(=O)N(C)[N+](=O)[O-]</smiles>
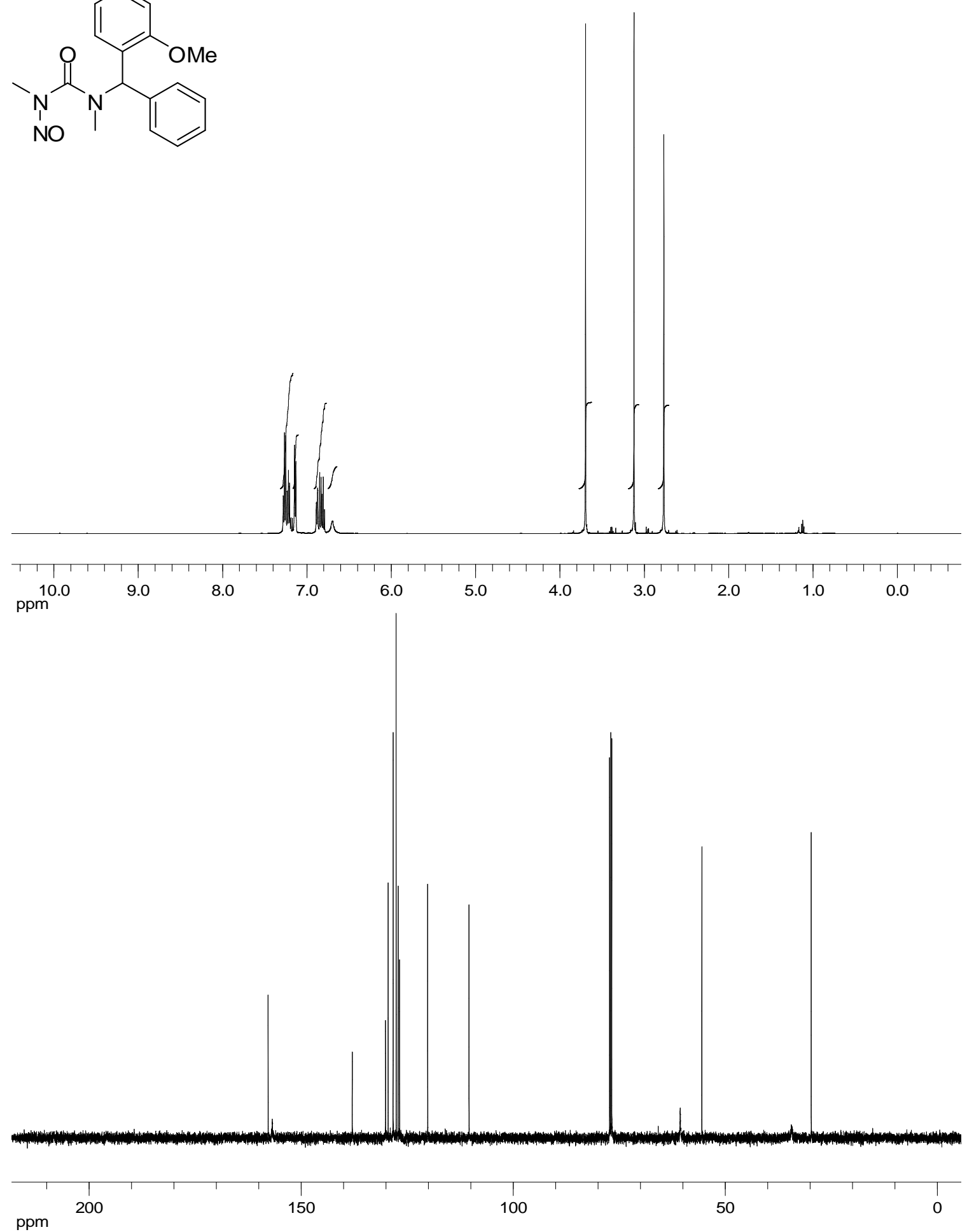
( \pm )-1-((2,6-Dimethylphenyl)benzyl)-1,3-dimethyl-3-nitrosourea $N$-nitroso-3g<smiles>Cc1cccc(C)c1C(c1ccccc1)N(C)C(=O)N(C)[N+](=O)[O-]</smiles>
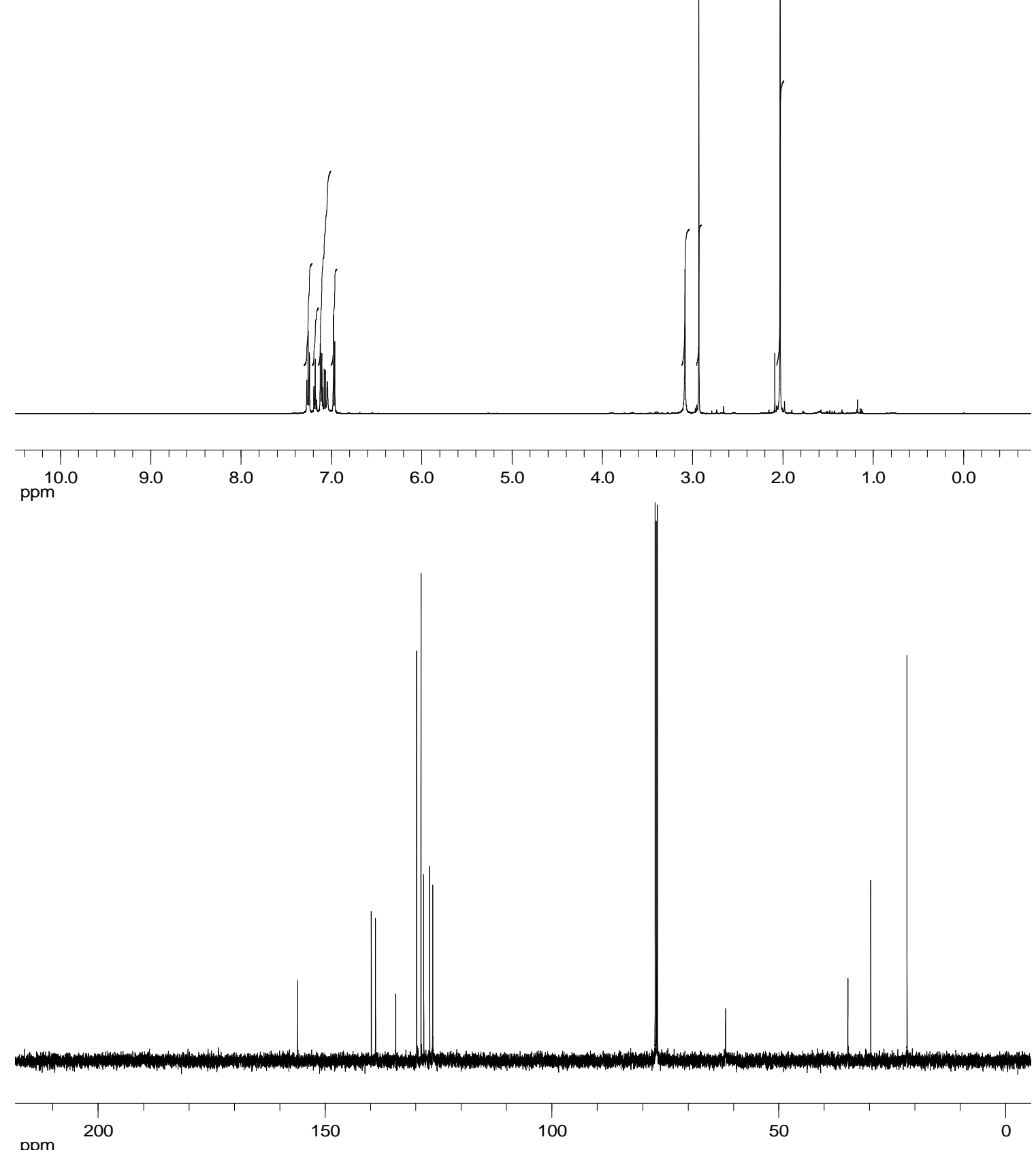

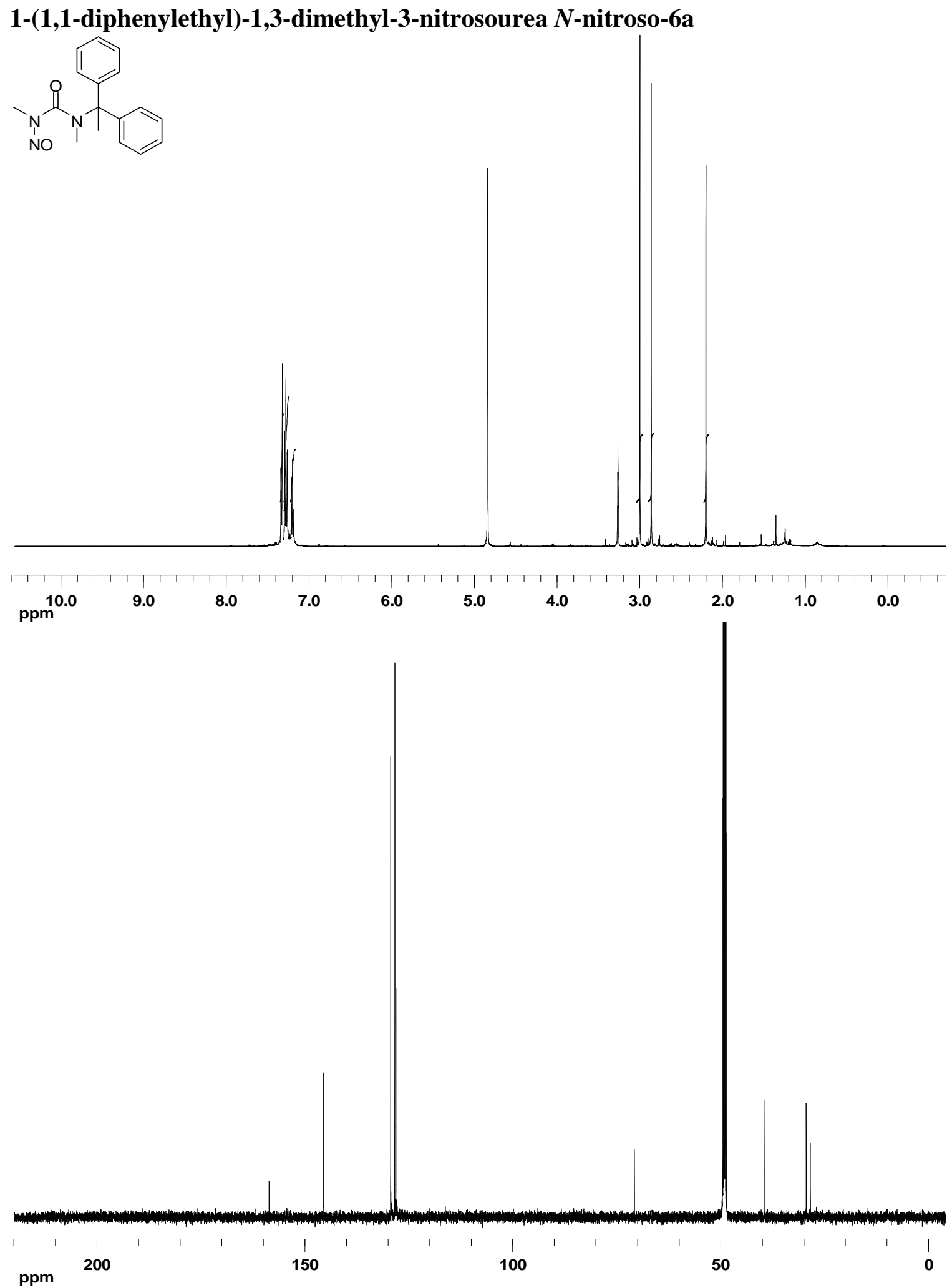
(S)-1-(1-(3-Chloro-4-fluorophenyl)-1-phenylethyl)-1,3-dimethyl-3-nitrosourea $N$ nitroso-6i
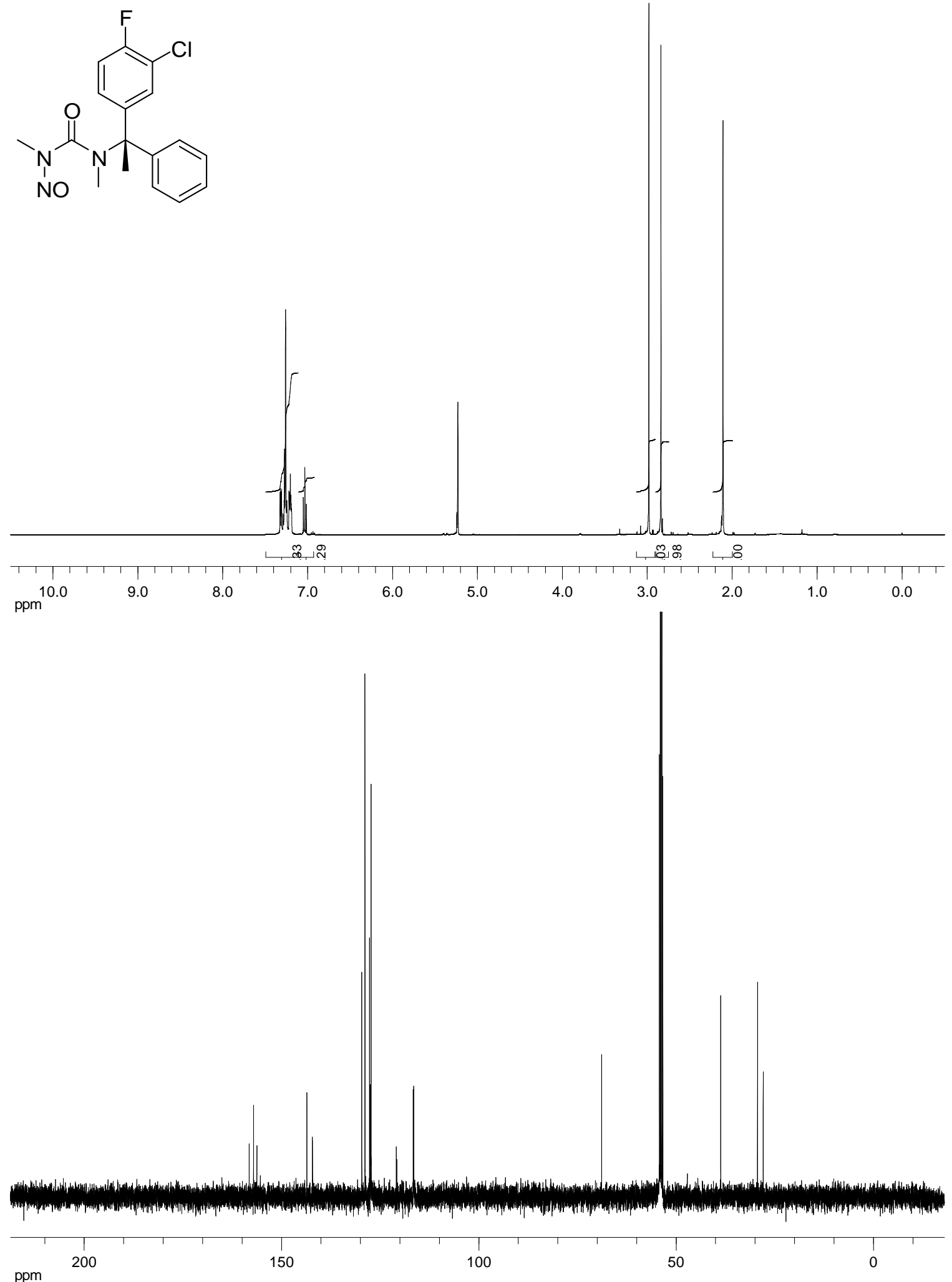
(S)-1,3-Dimethyl-1-(1-(naphthalen-1-yl)-1-phenylethyl)-3-nitrosourea $N$-nitroso-6j
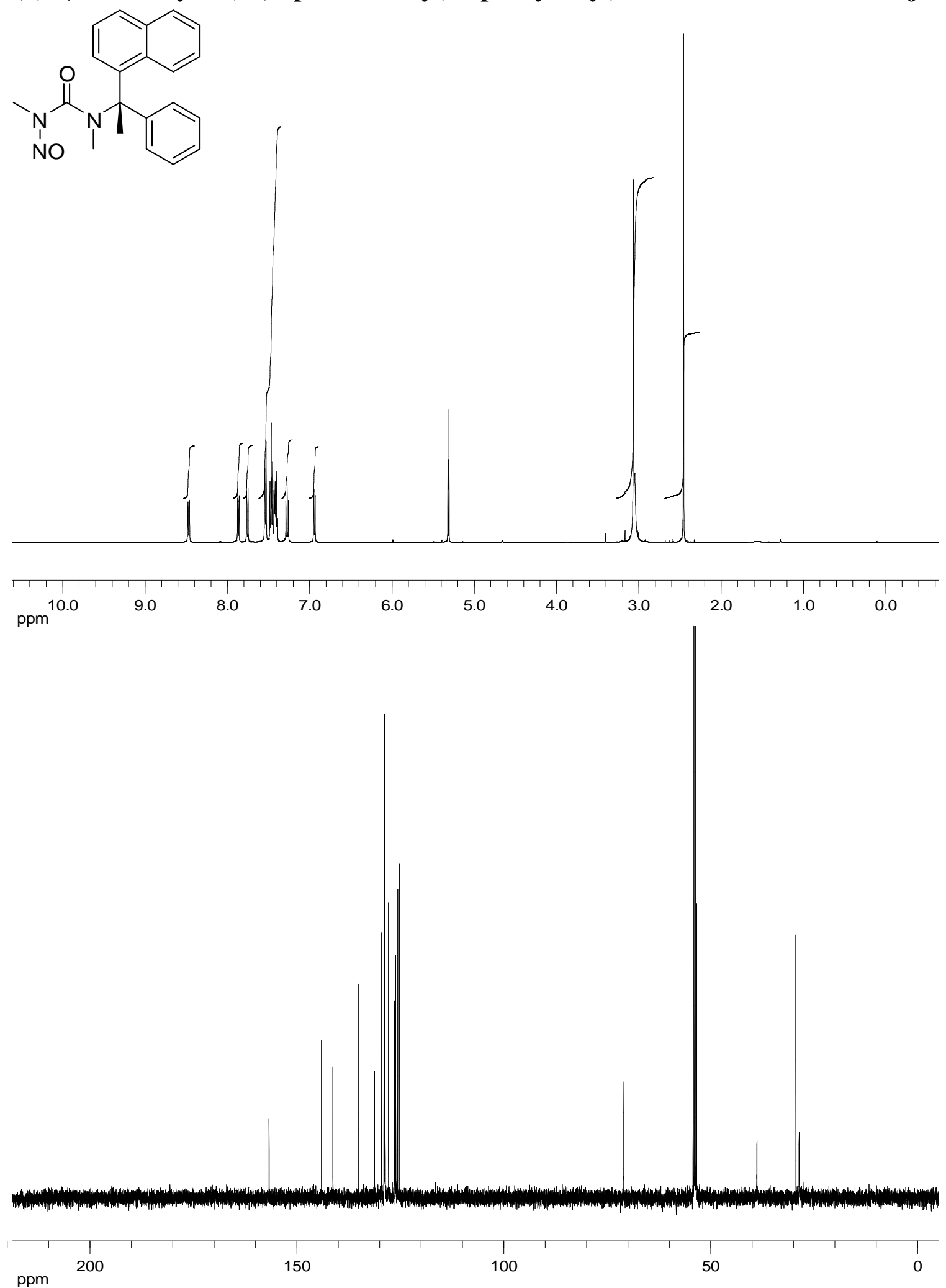


\section{HPLC Data}

\section{1-((R)-1-(4-Methoxyphenyl)-1-phenylethyl)-1,3-dimethylurea 6b}<smiles>CNC(=O)N(C)C(c1ccccc1)(c1ccccc1)c1ccc(OC)cc1</smiles>

Racemic

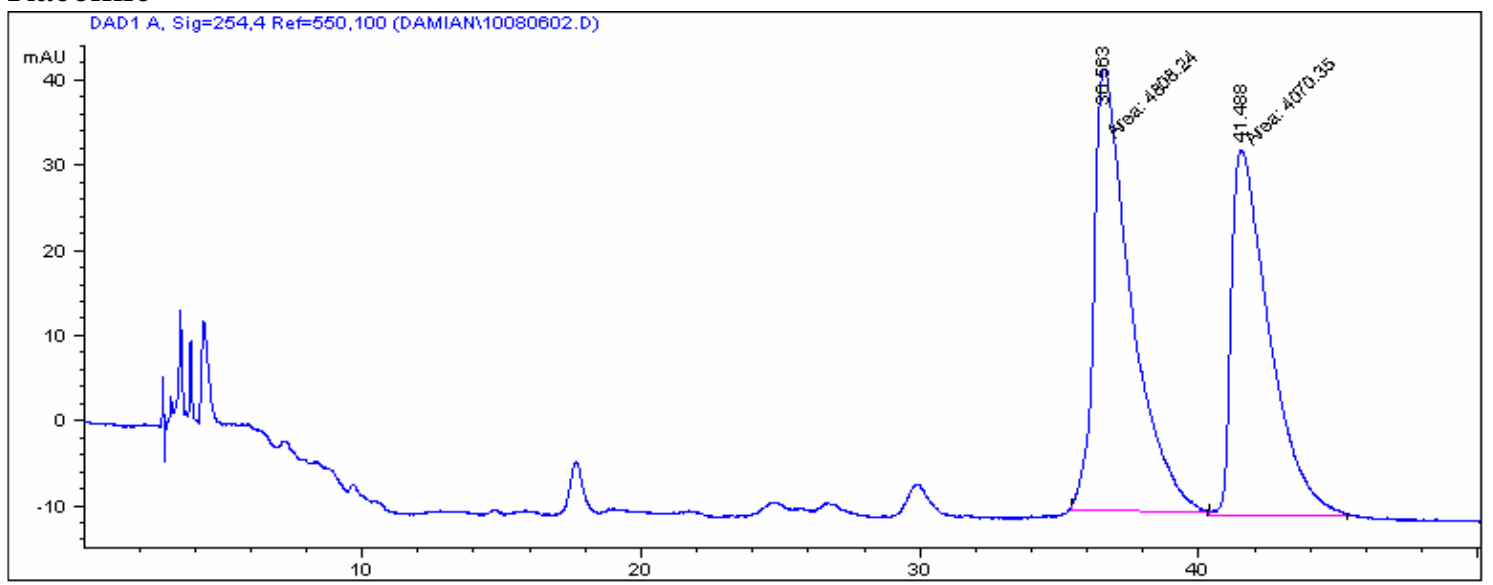

Enriched

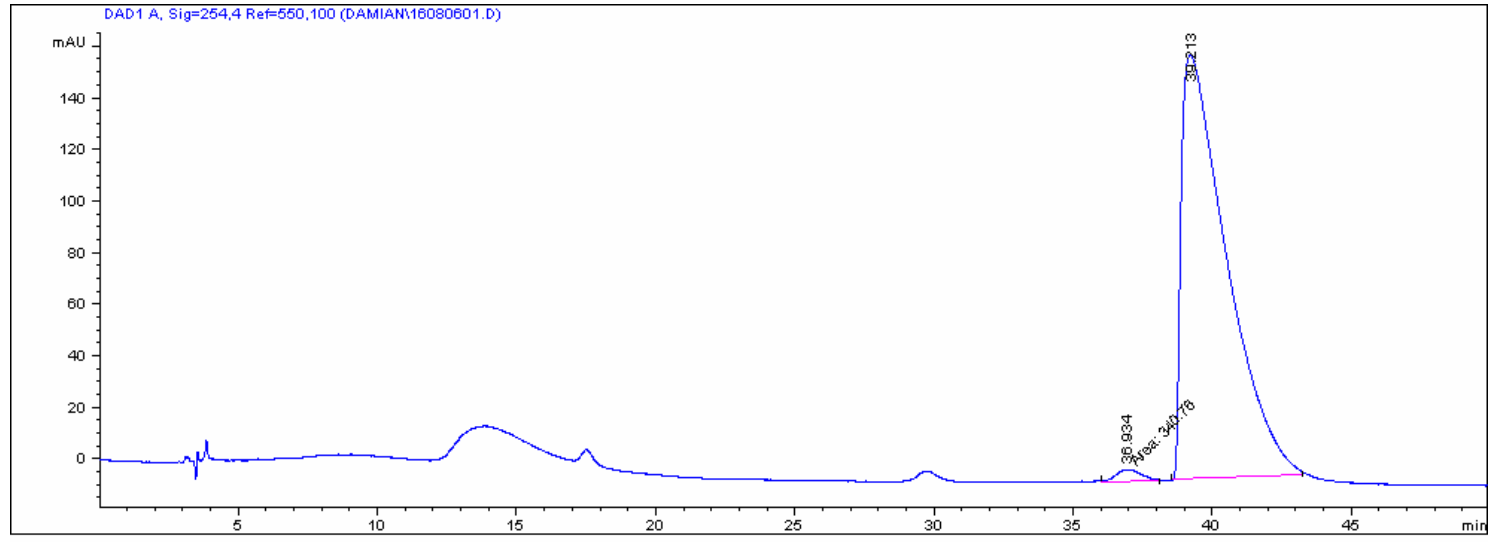


(R)-1-(1-(4-Methoxyphenyl)-1-phenylethyl)-1,3-dimethyl-3-nitrosourea 6b'<smiles>COc1ccc(C(c2ccccc2)N(C)C(=O)N(C)[N+](=O)[O-])cc1</smiles>

Racemic

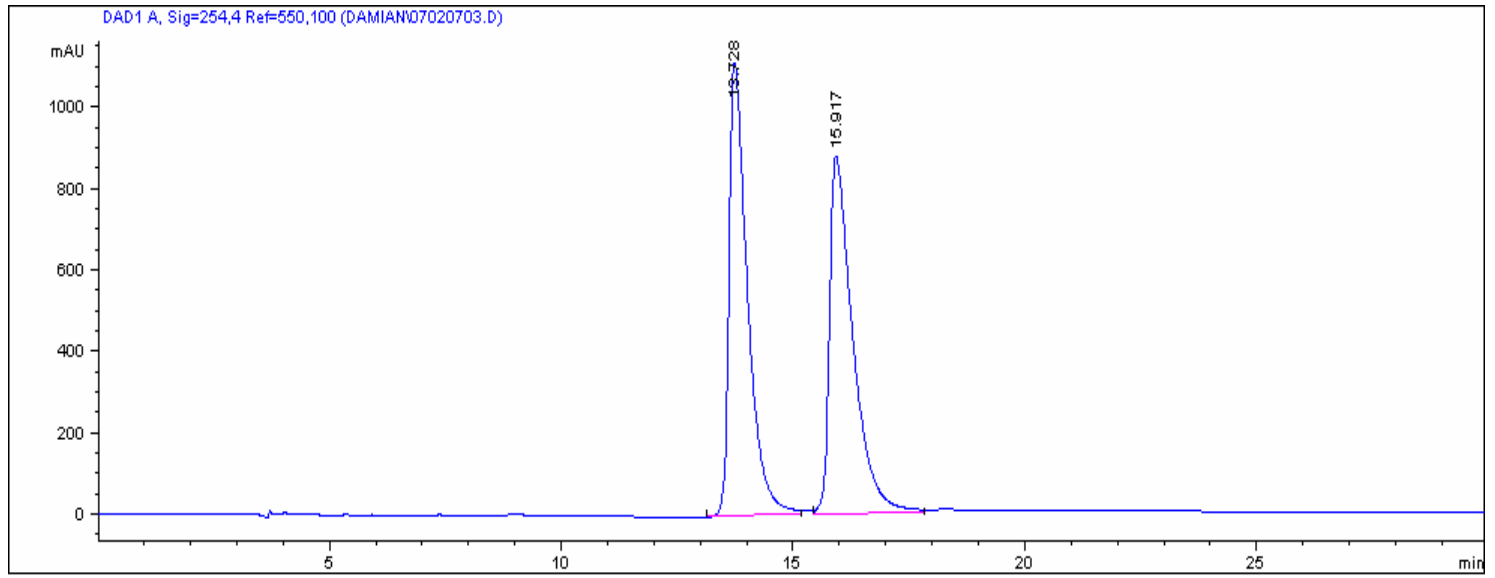

Enriched

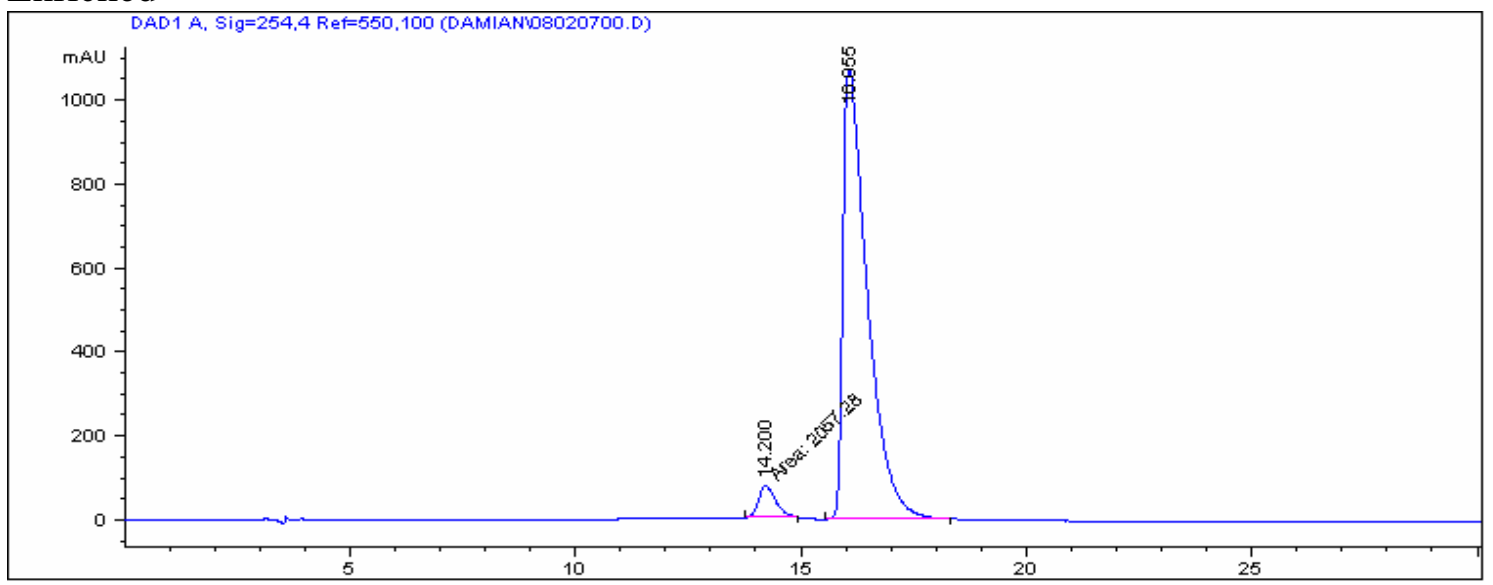




\section{1-((S)-1-(3,4-Dimethoxyphenyl)-1-phenylethyl)-1,3-dimethylurea 6d}<smiles>CNC(=O)N(C)[C@@](C)(c1ccccc1)c1ccc(OC)c(OC)c1</smiles>

Racemic

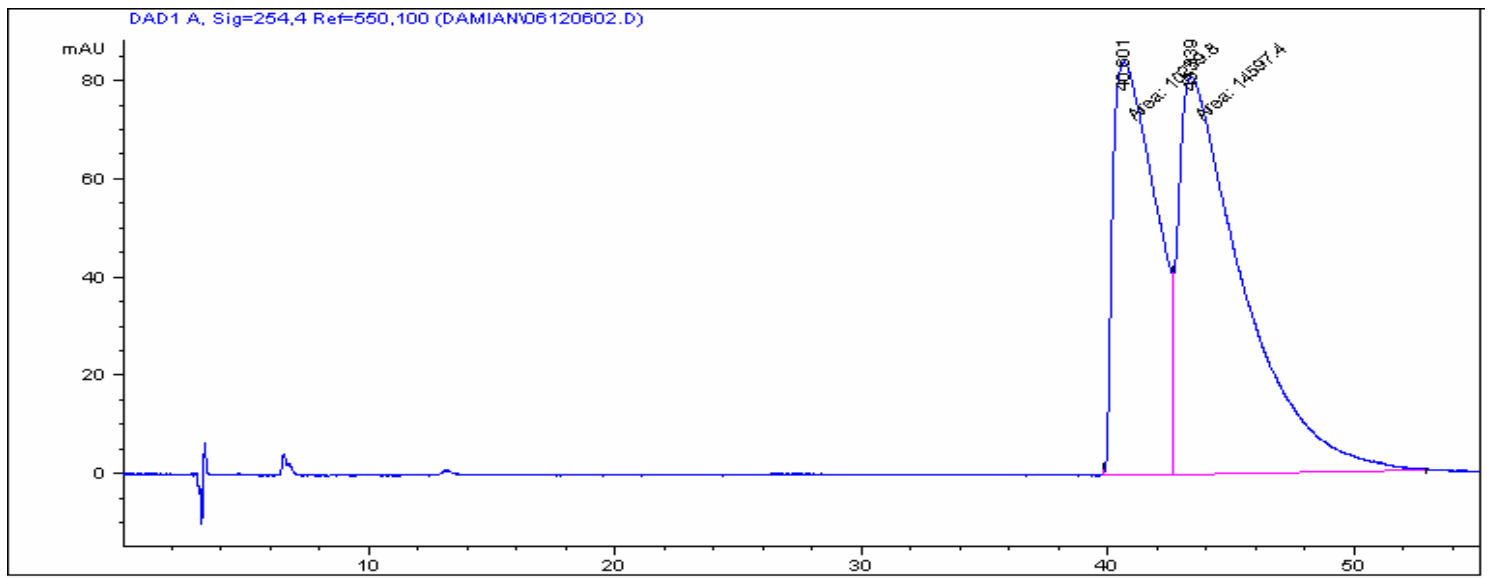

\section{Enriched}

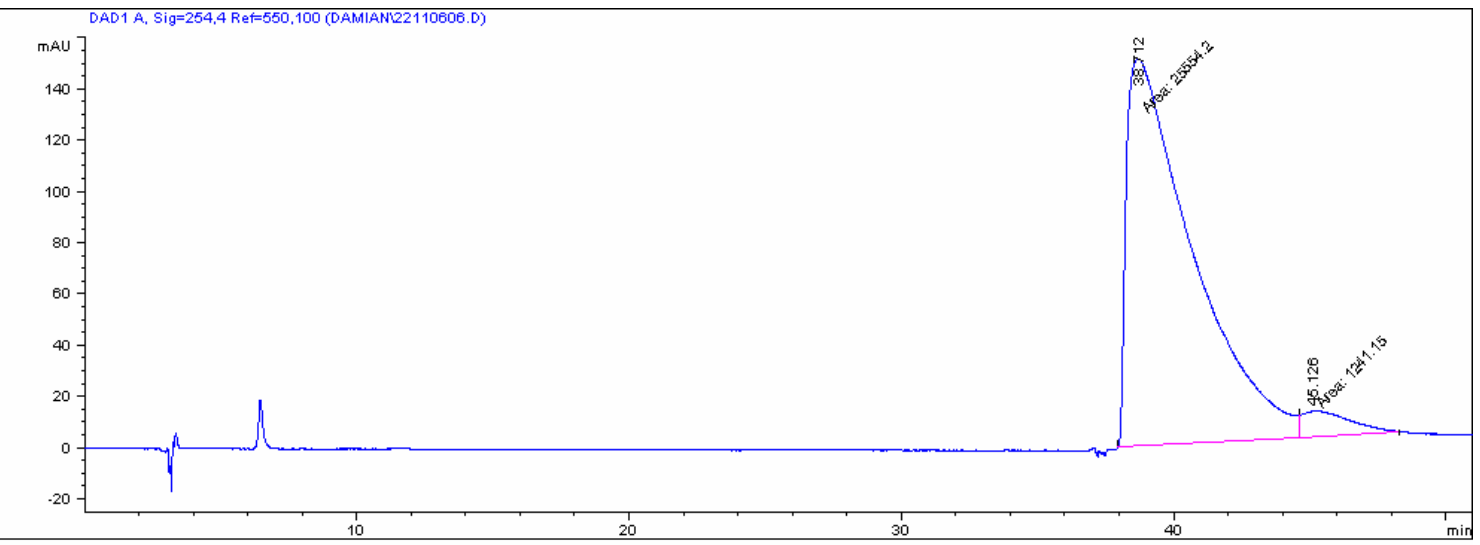




\section{1-((S)-1-(4-Fluorophenyl)-1-phenylethyl)-1,3-dimethylurea 6g}<smiles>CNC(=O)N(C)[C@@](C)(c1ccccc1)c1ccc(F)cc1</smiles>

Racemic

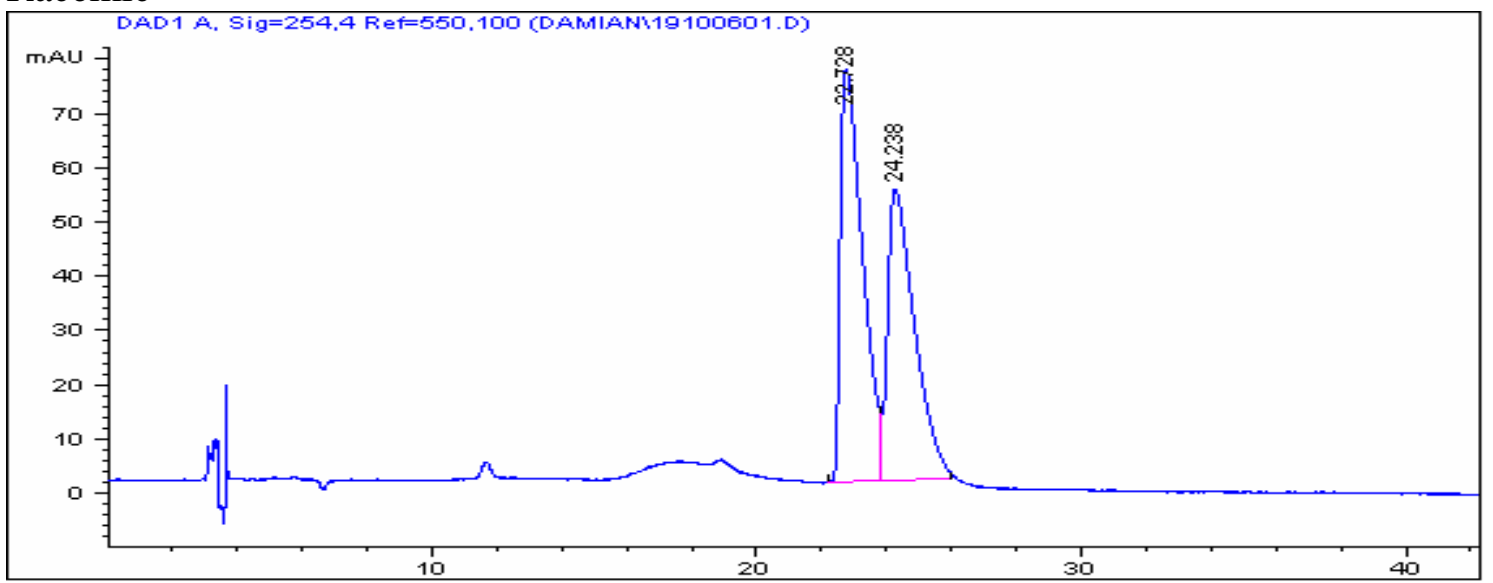

\section{Enriched}

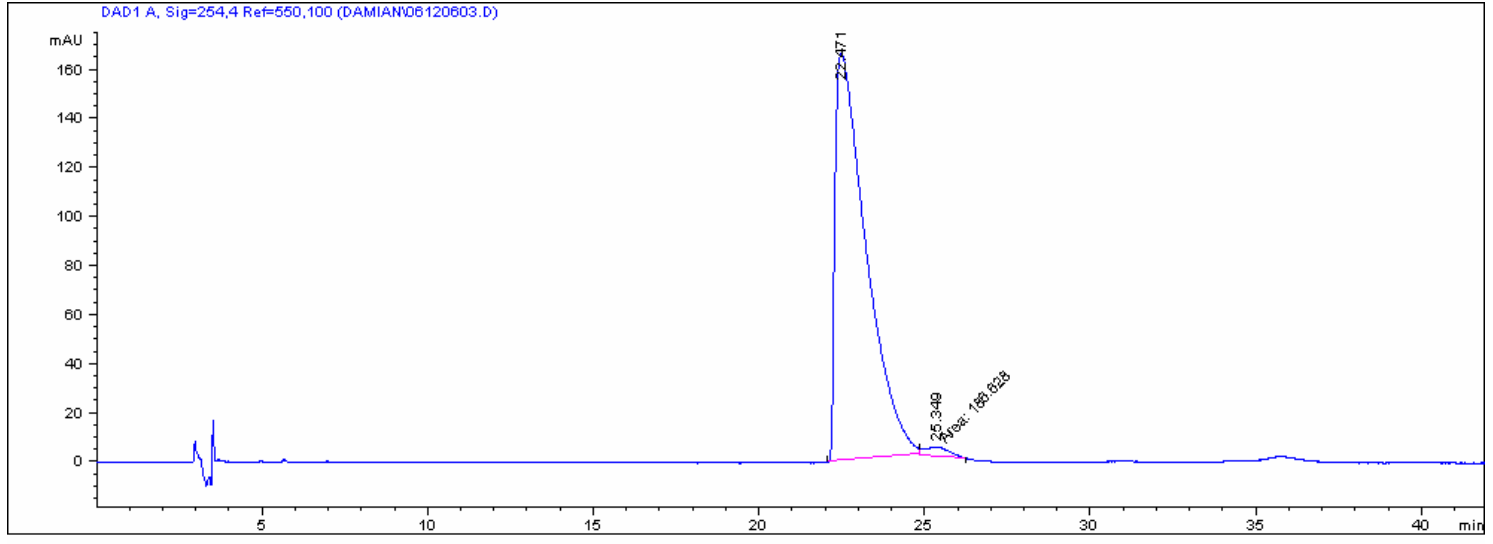




\section{1-((S)-1-(4-Chlorophenyl)-1-phenylethyl)-1,3-dimethylurea $6 \mathrm{~h}$}<smiles>CNC(=O)N(C)[C@@](C)(c1ccccc1)c1ccc(Cl)cc1</smiles>

Racemic

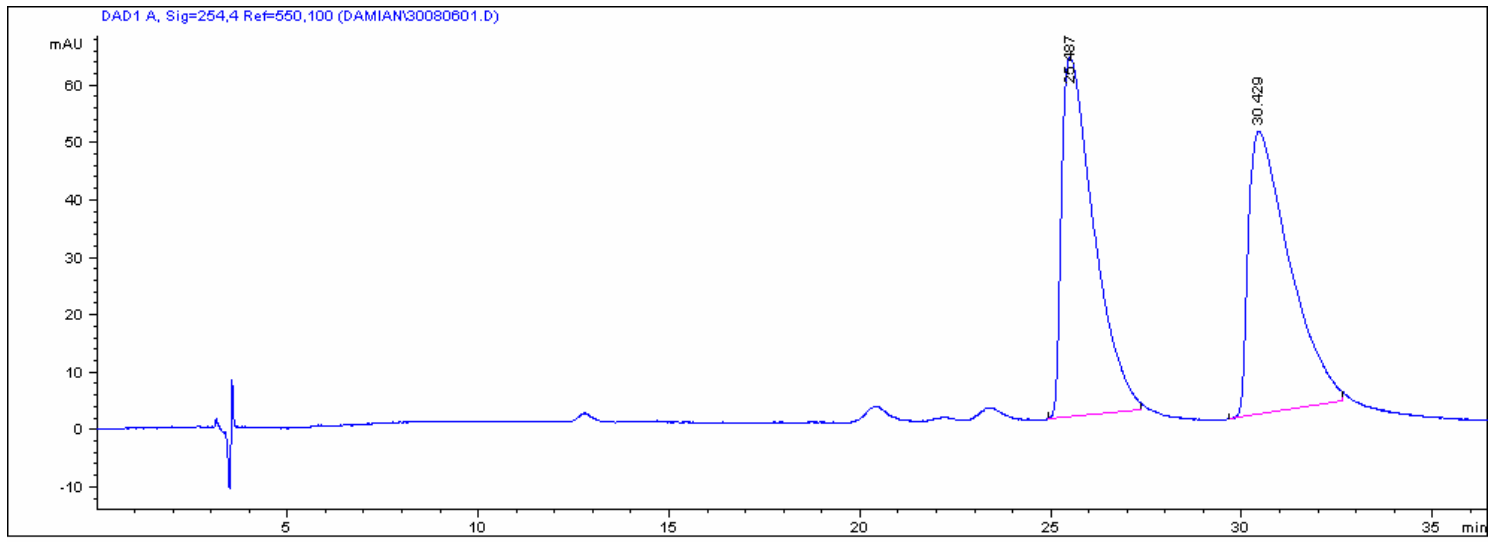

Enriched

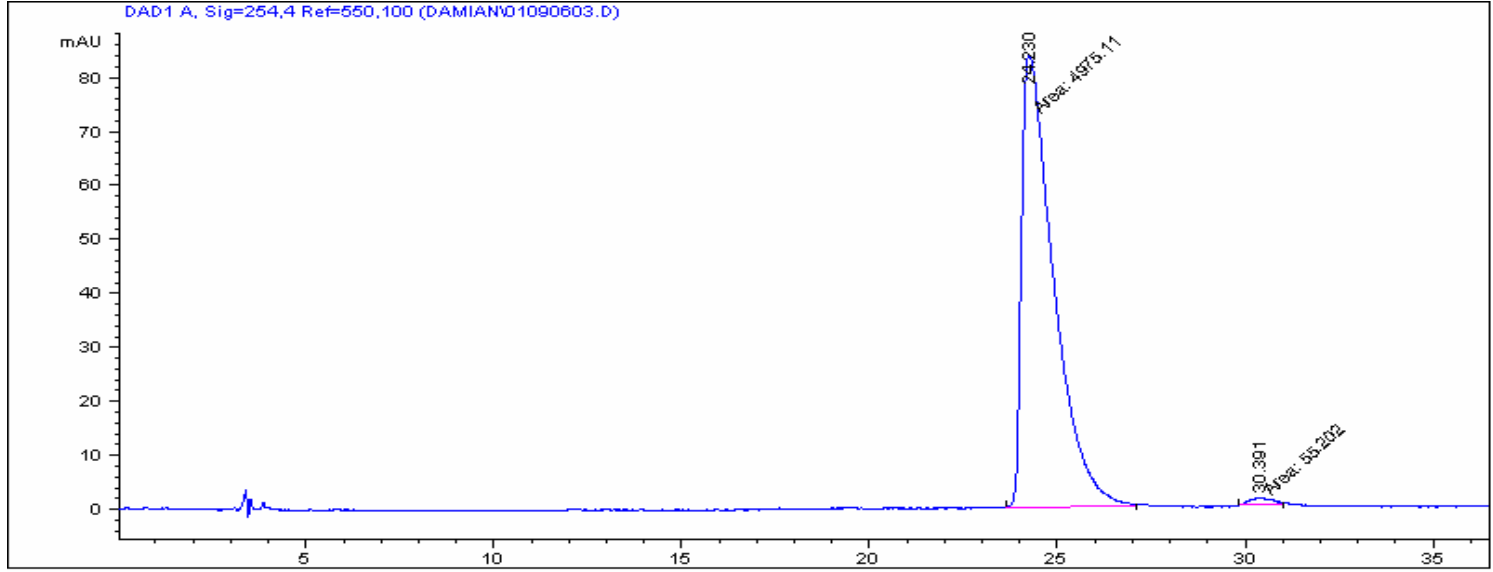




\section{1-((S)-1-(3-Chloro-4-fluorophenyl)-1-phenylethyl)-1,3-dimethylurea $6 \mathbf{i}$}<smiles>CNC(=O)N(C)[C@@](C)(c1ccccc1)c1ccc(F)c(Cl)c1</smiles>

Racemic

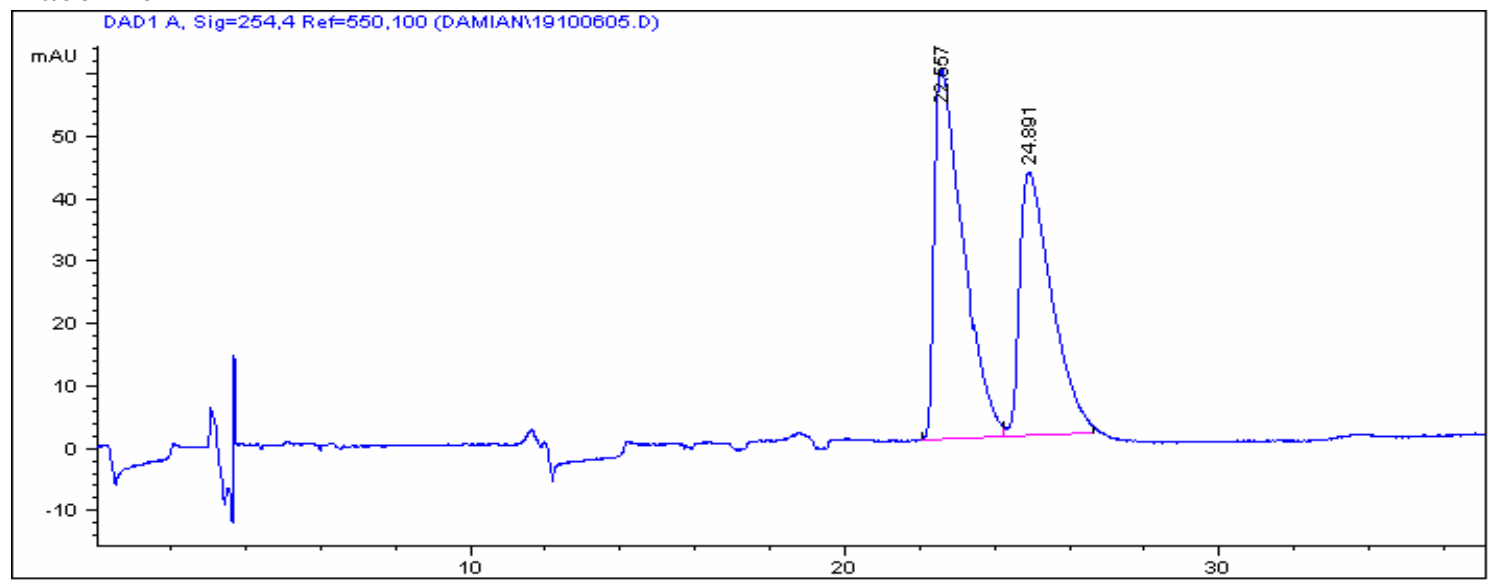

\section{Enriched}

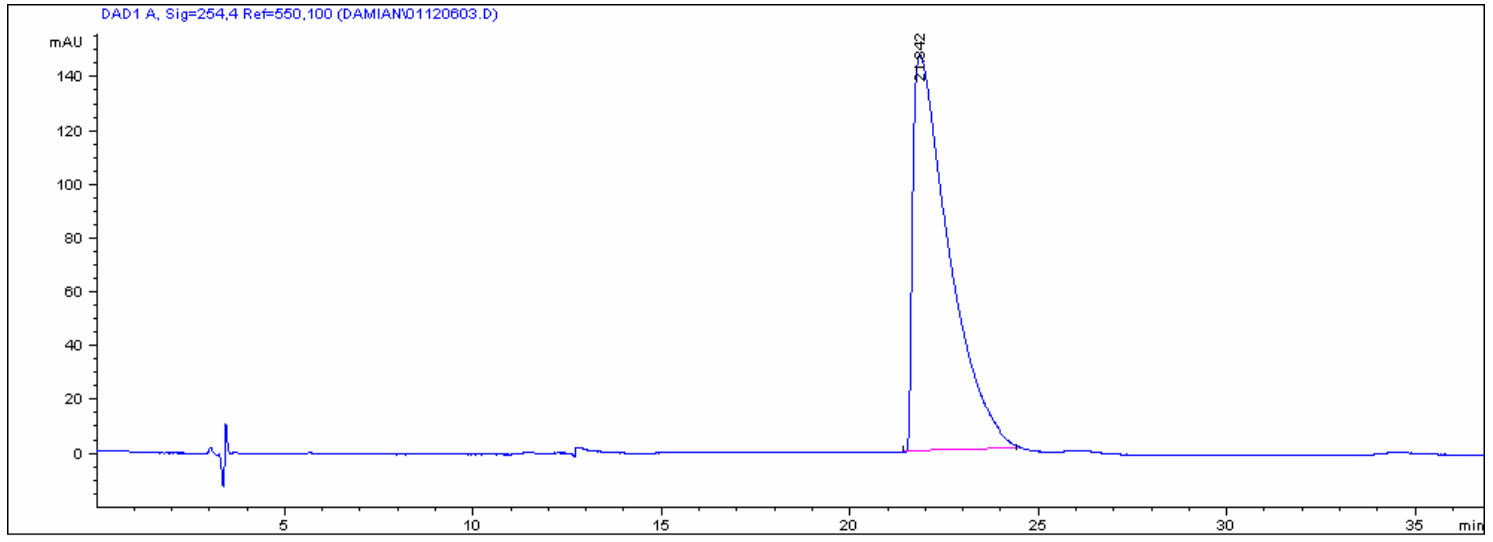




\section{1,1,3-Trimethyl-3-((S)-1-(naphthalen-1-yl)-1-phenylethyl)urea 6j}<smiles>CN(C)C(=O)N(C)[C@](C)(c1ccccc1)c1cccc2ccccc12</smiles>

\section{Racemic}

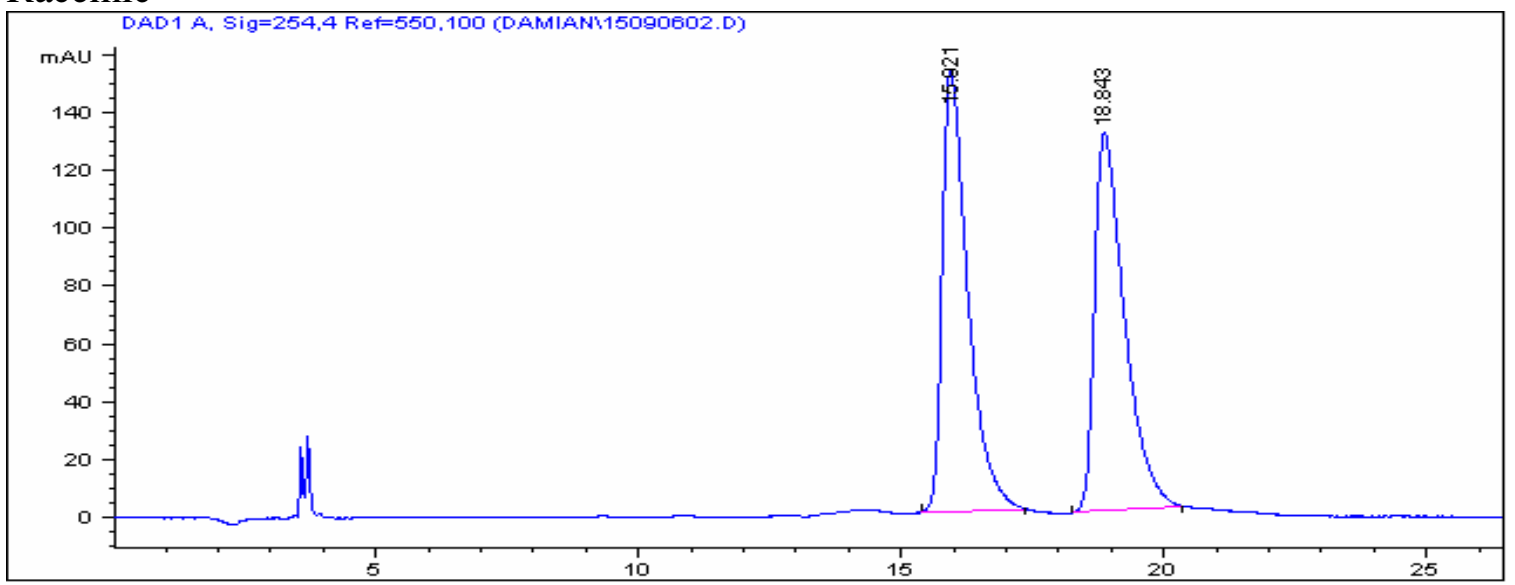

Enriched

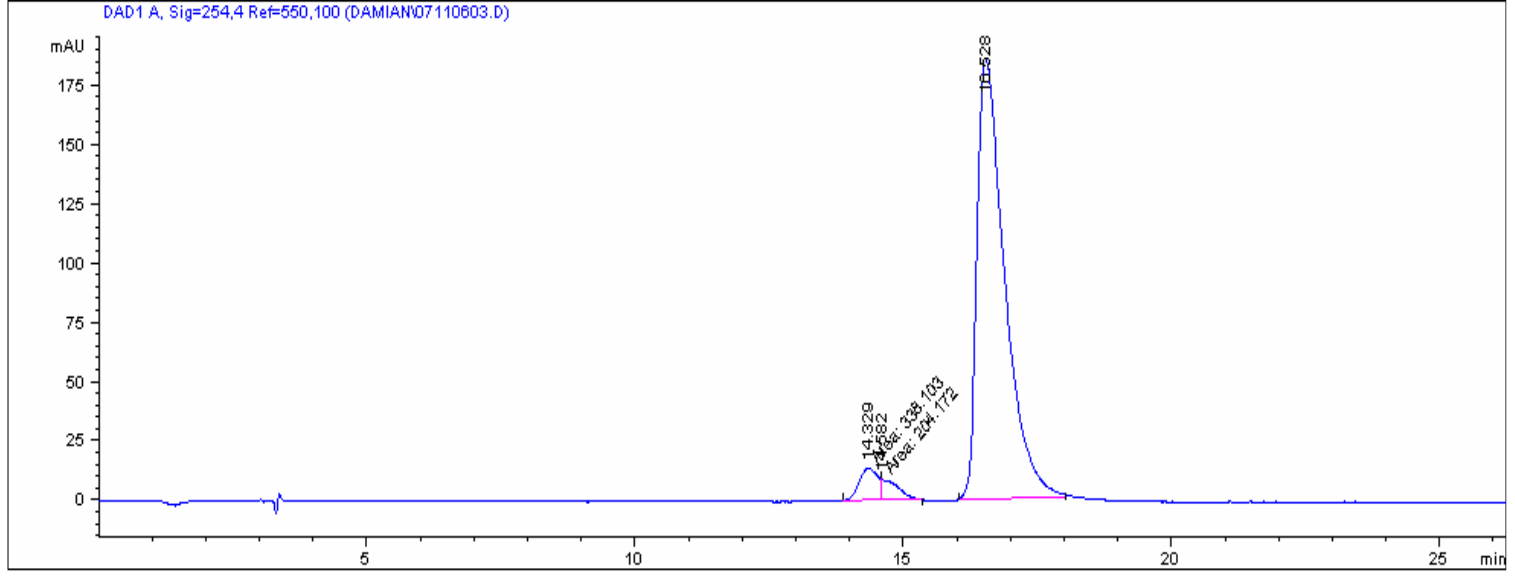




\section{1,1,3-Trimethyl-3-((S)-1-(naphthalen-2-yl)-1-phenylethyl)urea 6k}<smiles>CN(C)C(=O)N(C)[C@@](C)(c1ccccc1)c1ccc2ccccc2c1</smiles>

Racemic

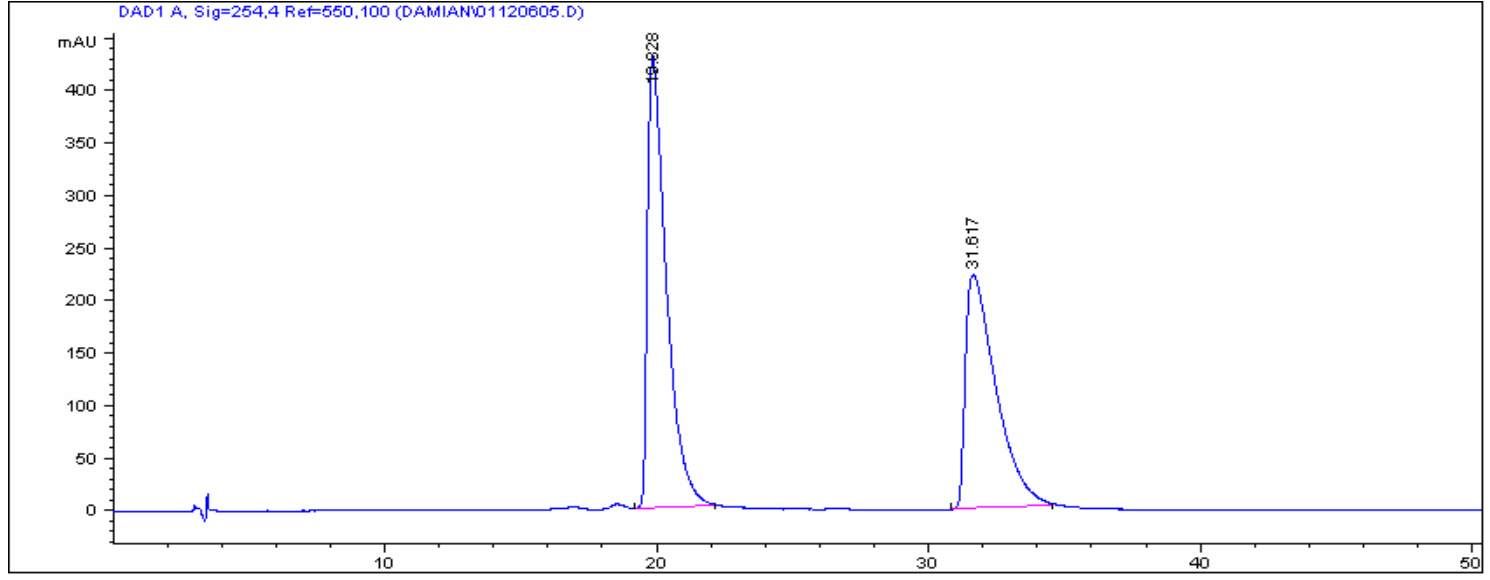

Enriched

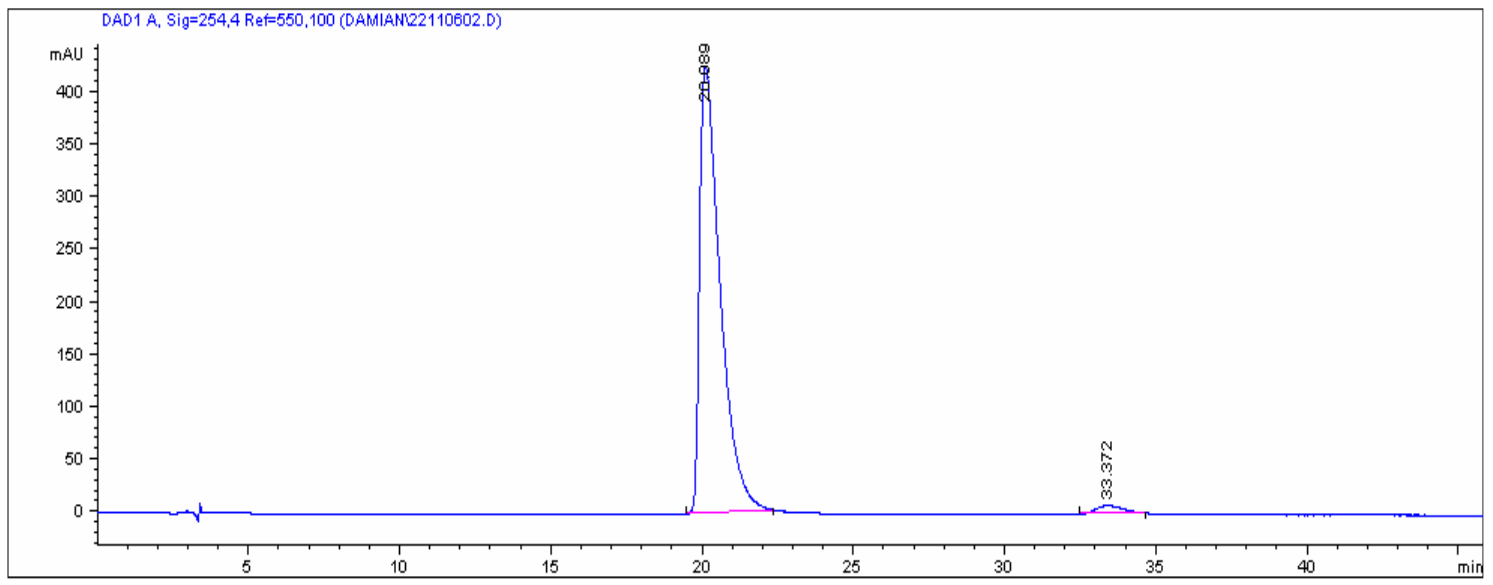




\section{1-((S)-1-(4-Methoxyphenyl)-1-o-tolylethyl)-1,3-dimethylurea 6m}<smiles>CNC(=O)N(C)[C@@](C)(c1ccc(OC)cc1)c1ccccc1C</smiles>

Racemic

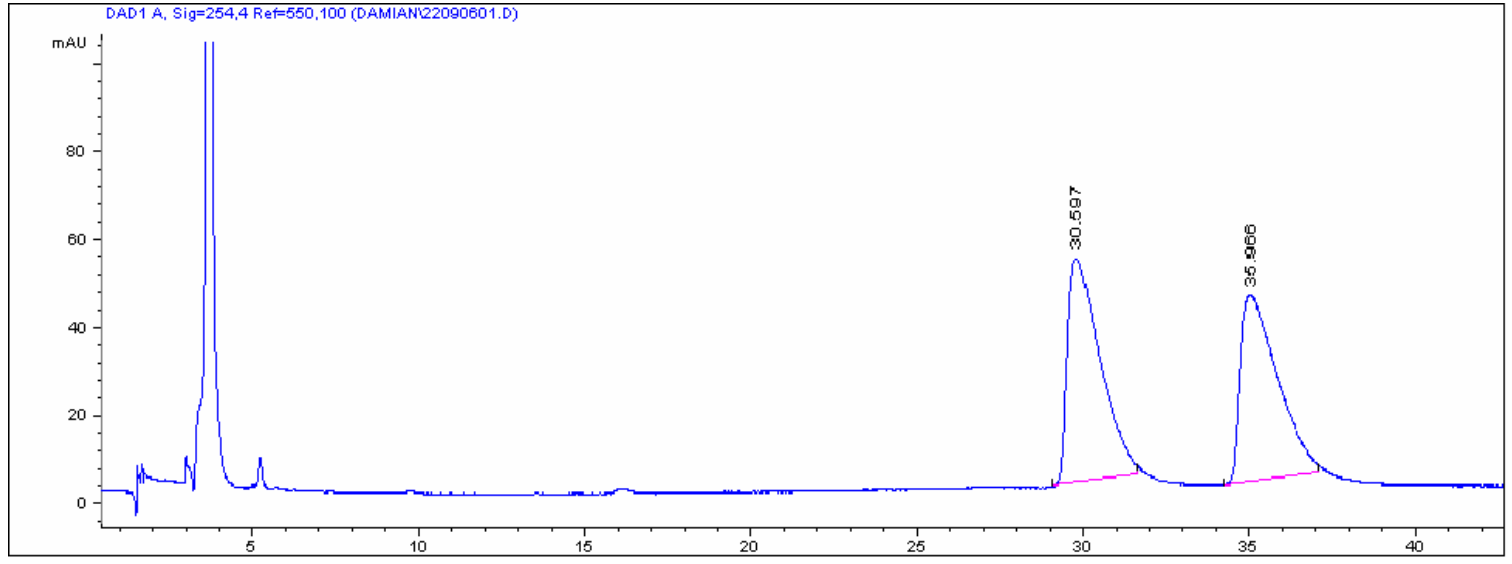

Enriched

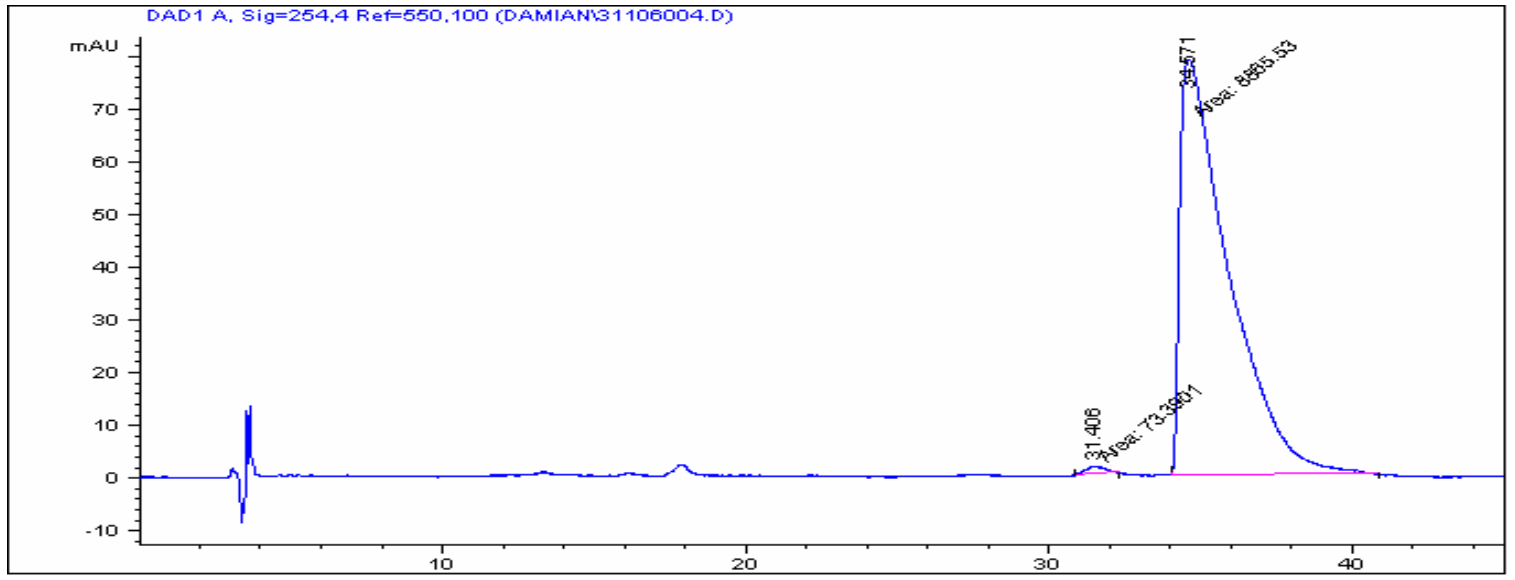




\section{1-((R)-1-(4-Methoxyphenyl)-1-p-tolylethyl)-1,3-dimethylurea 6n}<smiles>CNC(=O)N(C)C(C)(c1ccc(C)cc1)c1ccc(OC)cc1</smiles>

Racemic

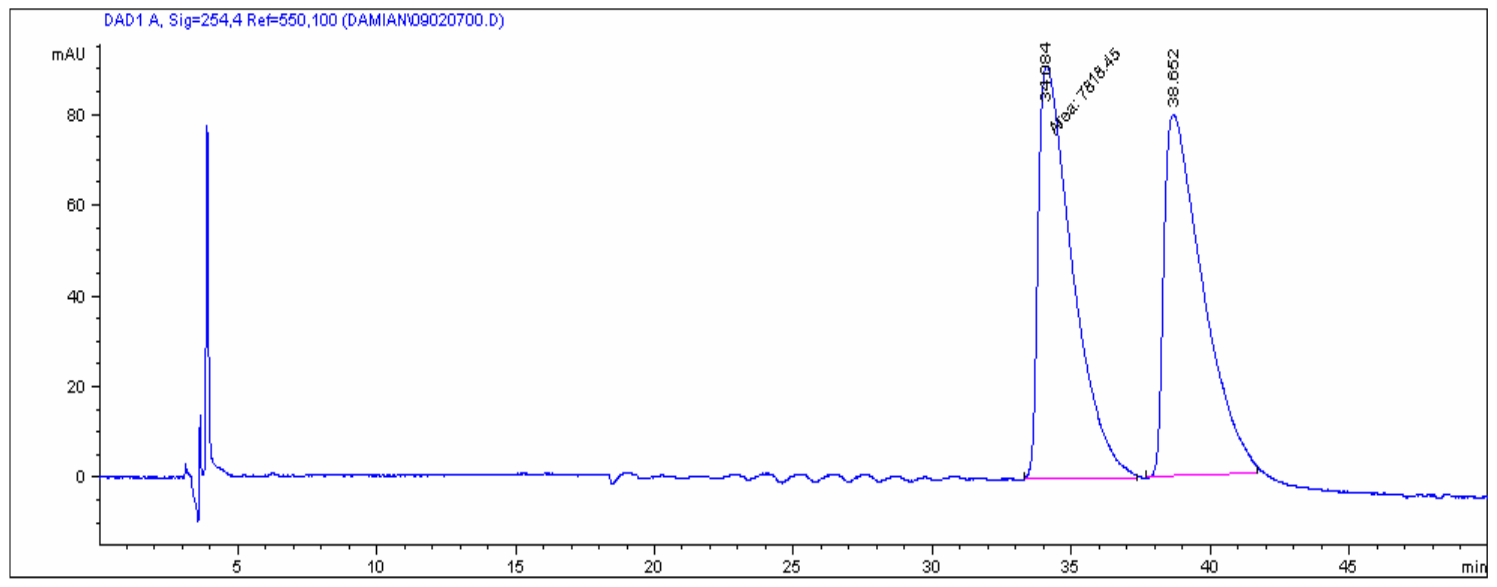

\section{Enriched}

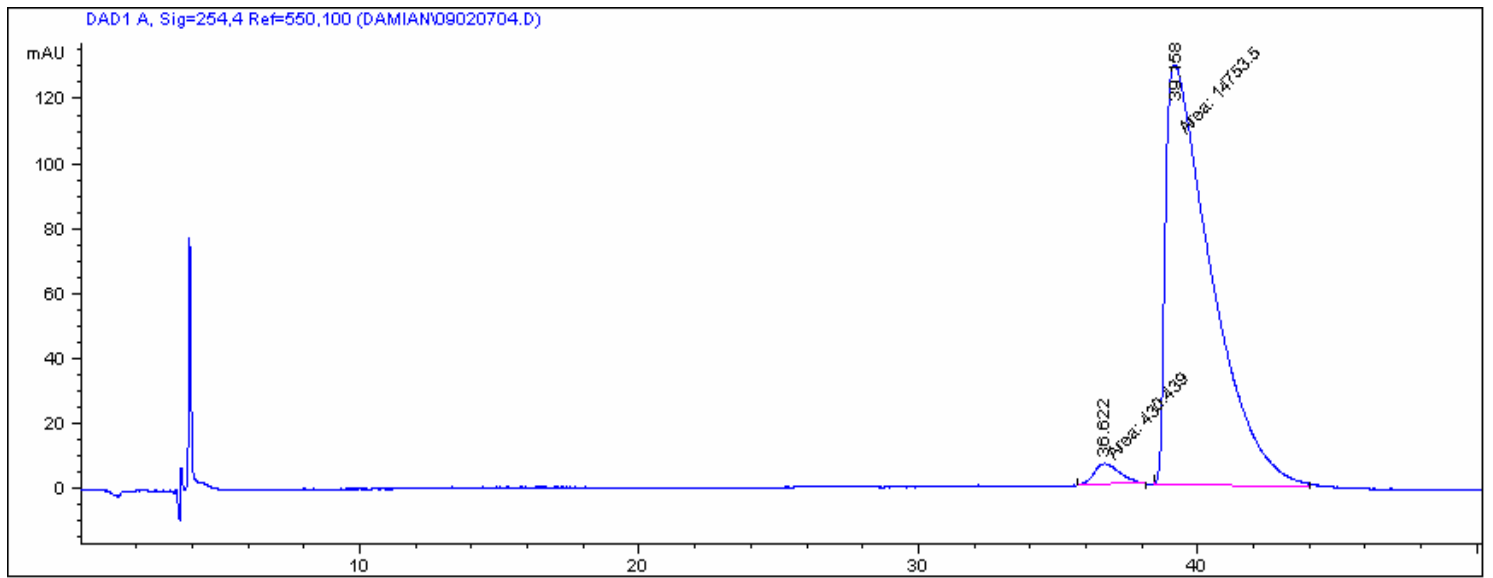




\section{1-((R)-1-(4-Methoxyphenyl)-1-(naphthalen-1-yl)ethyl)-1,3,3-trimethylurea 6o}<smiles>COc1ccc([C@H](c2cccc3ccccc23)N(C)C(=O)N(C)C)cc1</smiles>

\section{Racemic}

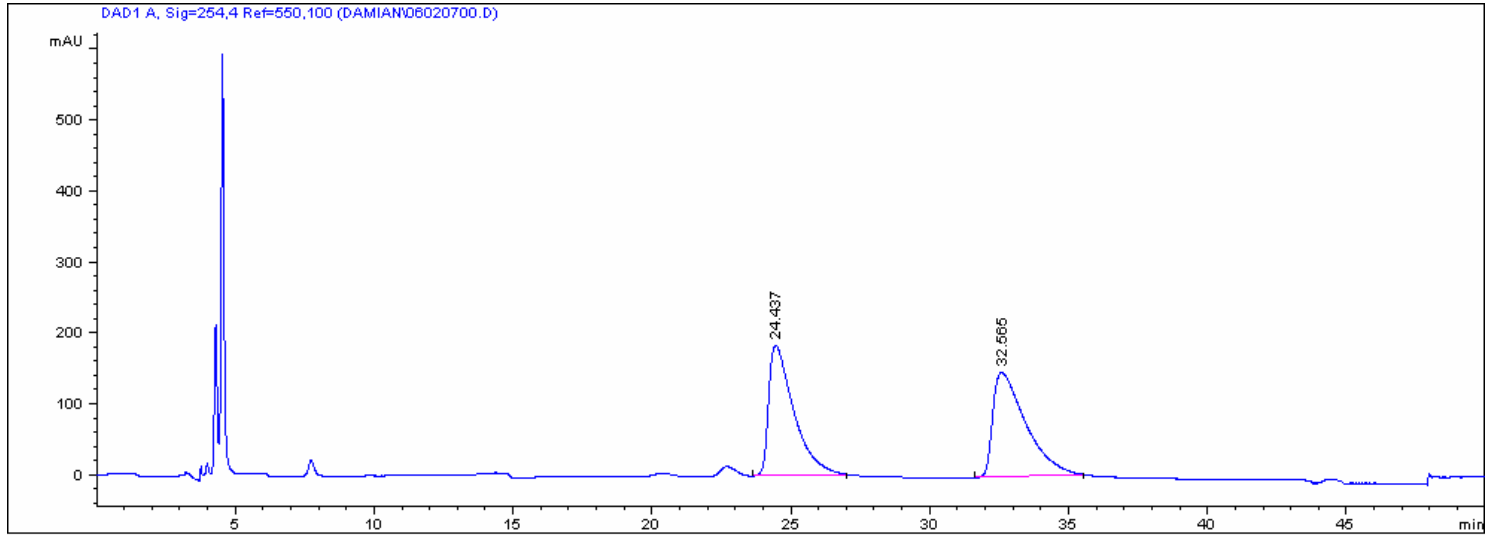

\section{Enriched}

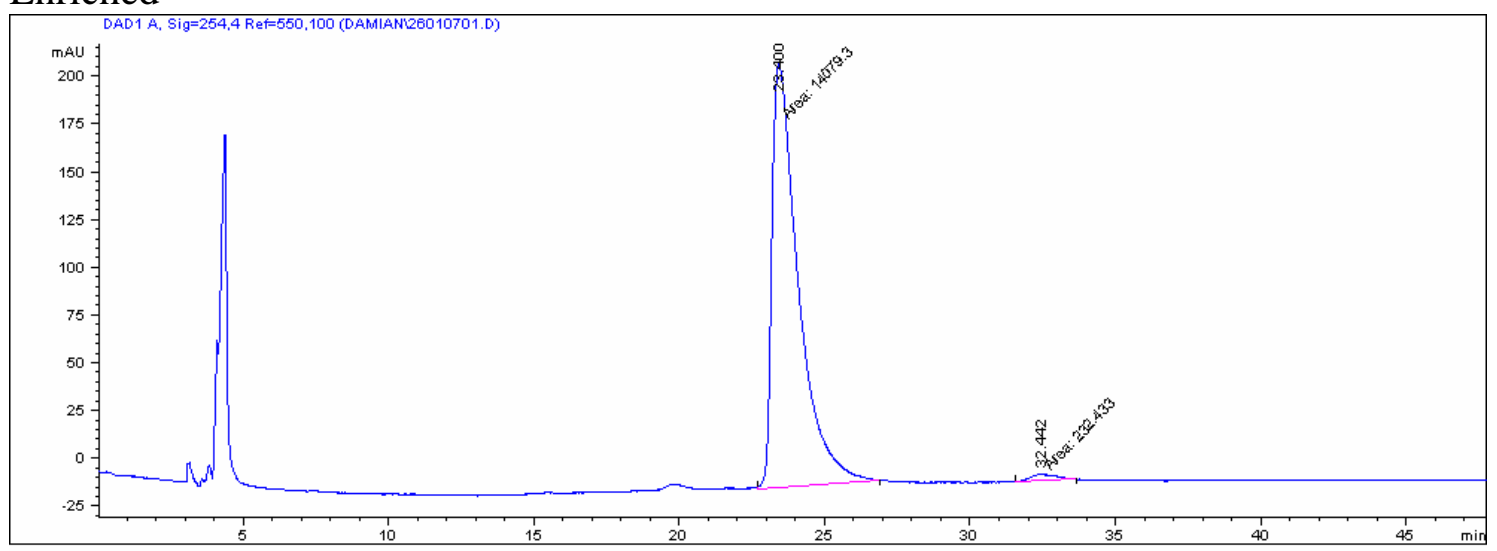




\section{1-((R)-1-(4-Chlorophenyl)-1-(naphthalen-1-yl)ethyl)-1,3,3-trimethylurea 6p}<smiles>CN(C)C(=O)N(C)[C@@](c1ccccc1)(c1ccc(Cl)cc1)c1cccc2ccccc12</smiles>

Racemic

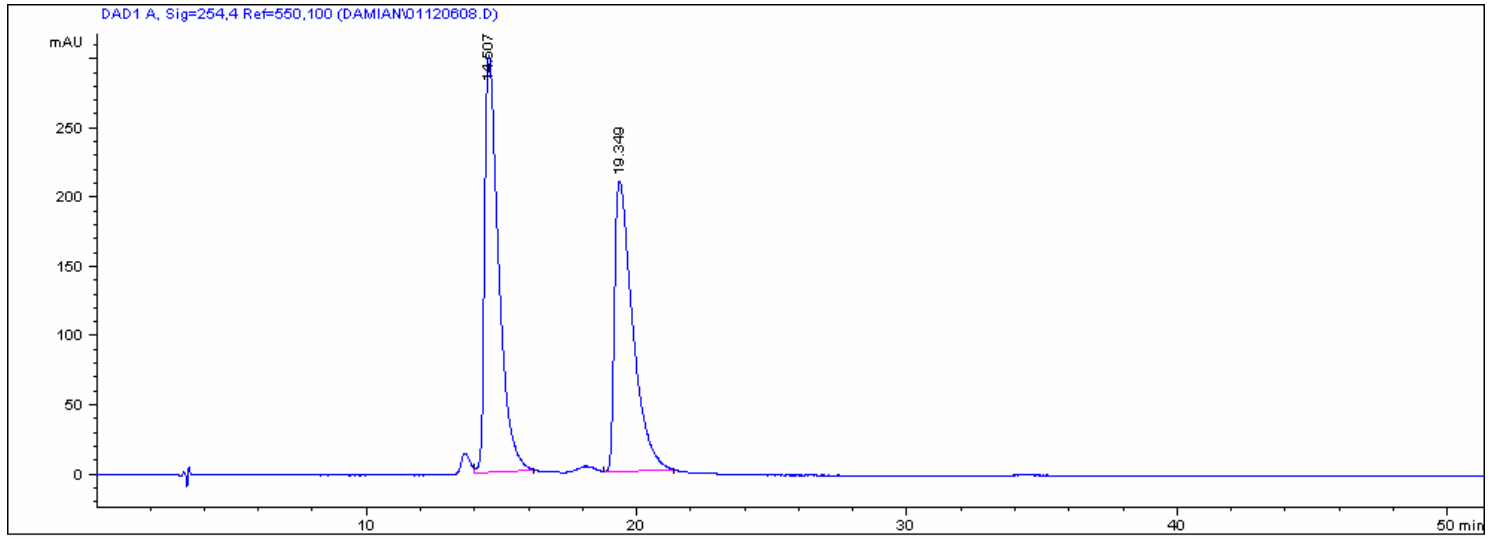

Enriched

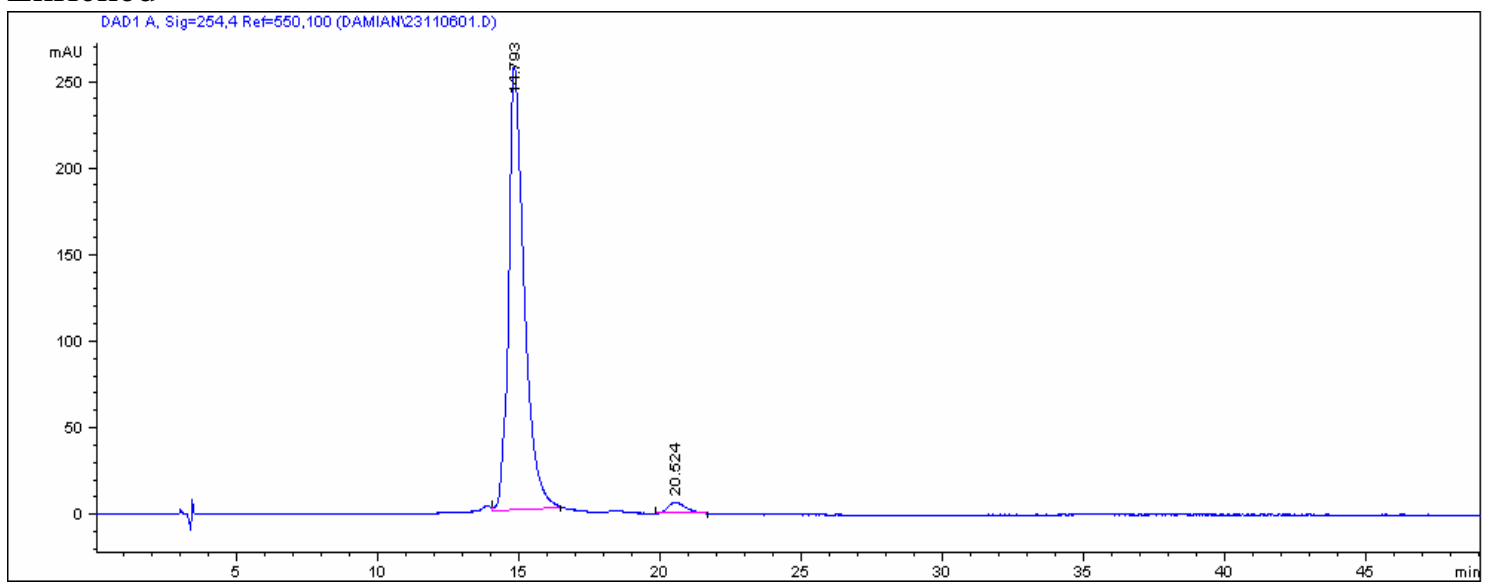


(S)-1,3-Dimethyl-1-(1-(naphthalen-1-yl)-1-phenylethyl)-3-nitrosourea $N$-nitroso-6j<smiles>CN(C(=O)N(C)[C@](C)(c1ccccc1)c1cccc2ccccc12)[N+](=O)[O-]</smiles>

Racemic

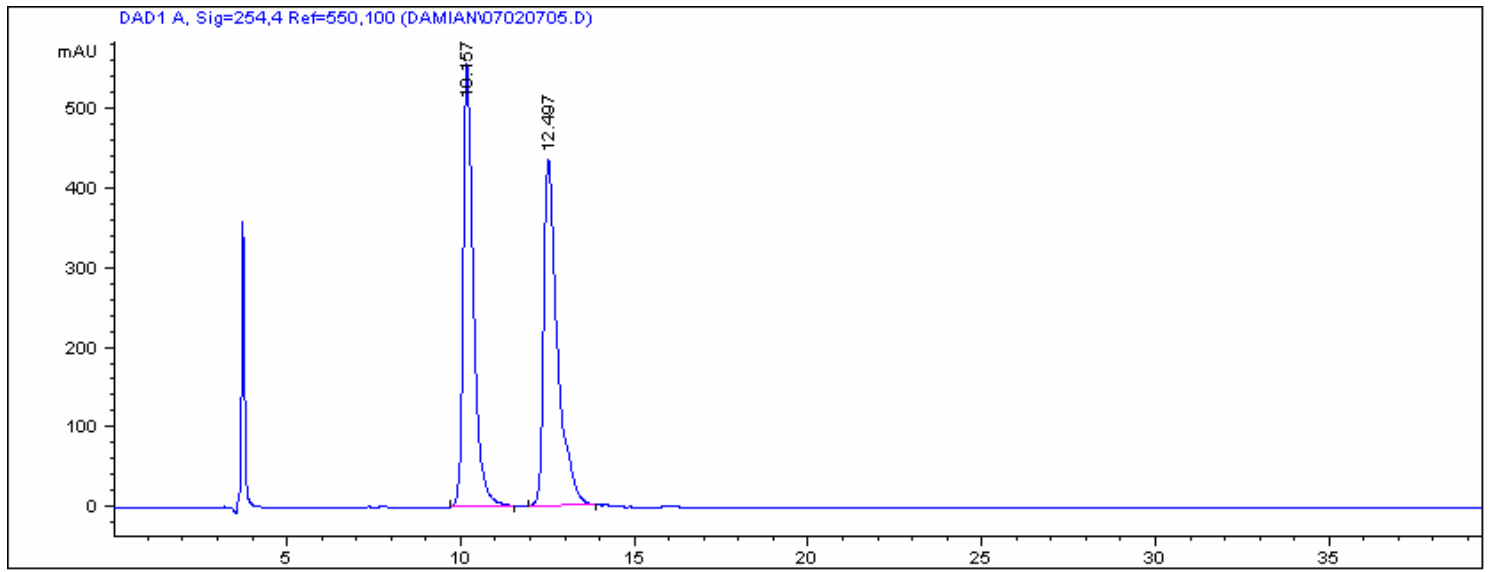

Enriched

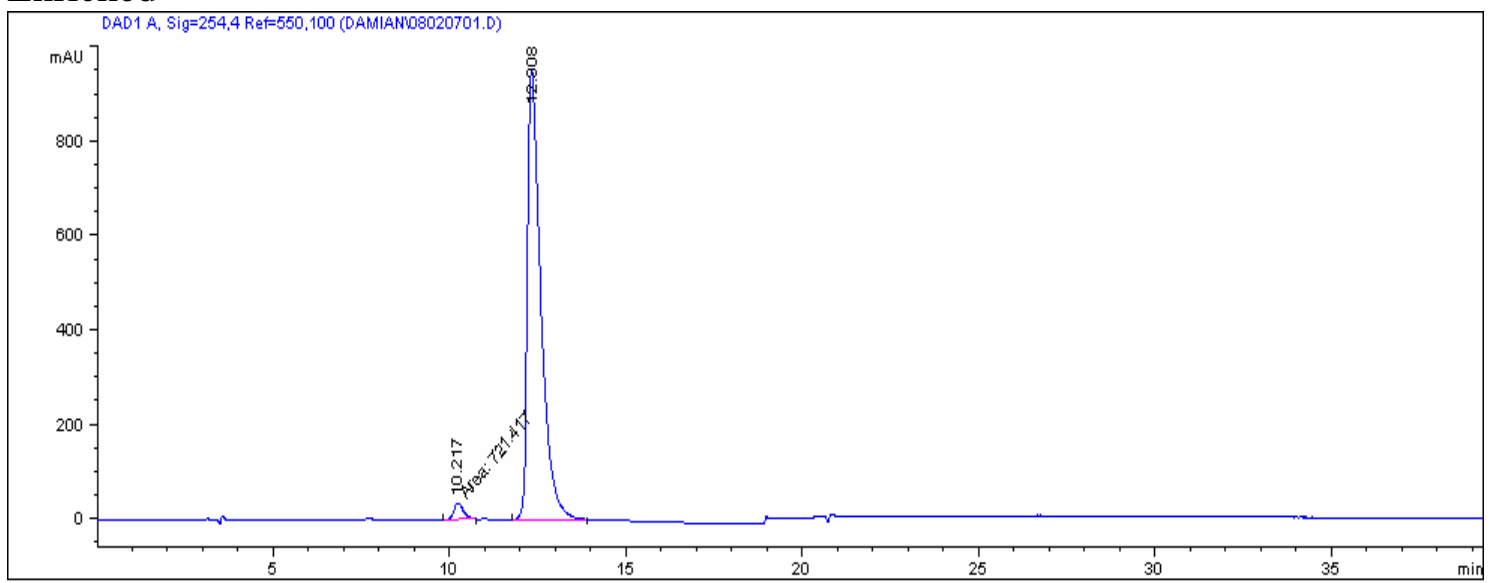

